\title{
Diagnostik und Veränderung des posttraumatischen Torsionsfehlers nach suprakondylären Humerusfrakturen im Wachstumsalter
}

\author{
INAUGURAL-DISSERTATION \\ zur Erlangung des Doktorgrades \\ der Medizinischen Fakultät der \\ Georg-August-Universität zu Göttingen \\ vorgelegt von \\ Claudia Cornelia Gadomski (geb. Rudolph) \\ aus \\ Kassel
}

Göttingen 2018 
Dekan:

Referent/in

Ko-Referent/in:

Drittreferent/in:
Prof. Dr. rer. nat. H. K. Kroemer

Prof. Dr. med. A. K. Hell

Prof. Dr. med. K. Dresing

Datum der mündlichen Prüfung: 10.10.2018 
Hiermit erkläre ich, die Dissertation mit dem Titel "Diagnostik und Veränderung des posttraumatischen Torsionsfehlers nach suprakondylären Humerusfrakturen im Wachstumsalter" eigenständig angefertigt und keine anderen als die von mir angegebenen Quellen und Hilfsmittel verwendet zu haben.

Göttingen, den

(Unterschrift) 
Teile dieser Dissertation wurden bereits veröffentlicht:

Katthagen JC: Humerustorsion bei Kindern und Jugendlichen mit spastischer Hemiparese. Med. Diss. Göttingen 2008

Katthagen JC, Rudolph C, Hell AK (2009): Sonographische Humerustorsionsmessungen bei Kindern und Jugendlichen mit spastischer Hemiparese. Orthopäde 38(10), 956-961 


\section{Inhaltsverzeichnis}

Abbildungsverzeichnis .........................................................................................

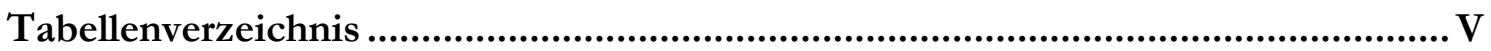

Abkürzungsverzeichnis.................................................................................... VII

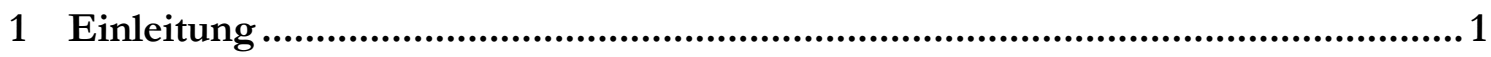

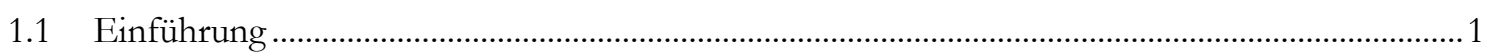

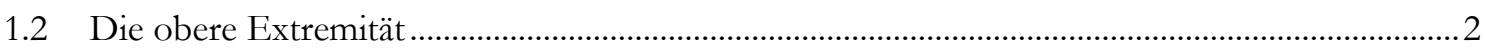

1.2.1 Anatomie des Humerus sowie des Schulter- und Ellenbogengelenkes.......................................2

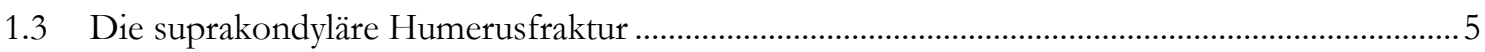

1.3.1 Pathophysiologie der suprakondylären Humerusfrakturen.......................................................

1.3.2 Klassifikation der suprakondylären Humerusfrakturen............................................................ 6

1.3.3 Diagnostik der suprakondylären Humerusfrakturen.................................................................

1.3.4 Therapie der suprakondylären Humerusfraktur....................................................................... 10

1.3.5 Komplikationen nach suprakondylären Humerusfrakturen ...................................................12

1.3.6 Der posttraumatische Rotationsfehler und seine Entwicklung am Humerus............................13

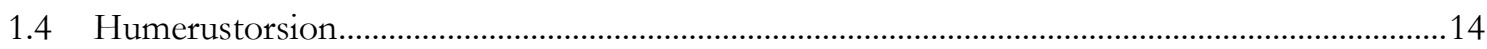

1.4.1 Definition der Humerustorsion/ Rotation ............................................................................14

1.4.2 Bedeutung der Humerustorsion für den Oberarm...............................................................

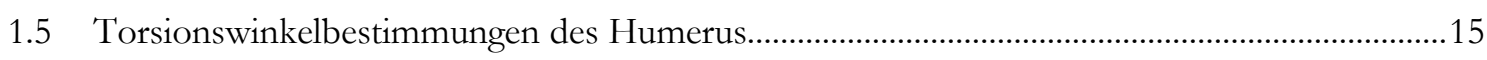

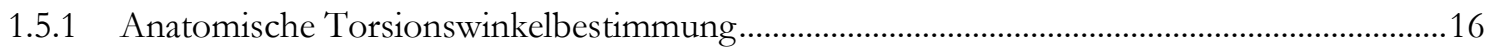

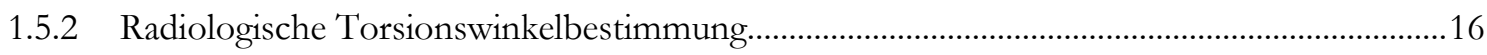

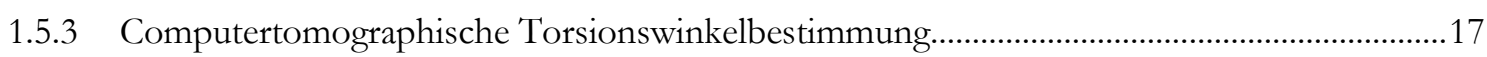

1.5.4 Sonographische Bestimmung des Torsionswinkels.....................................................................17

1.5.5 Verschiedene Messmethoden zur Bestimmung der Humerustorsion im Vergleich ................18

1.6 Entwicklung der femoralen Antetorsion im Wachstumsalter.........................................................19

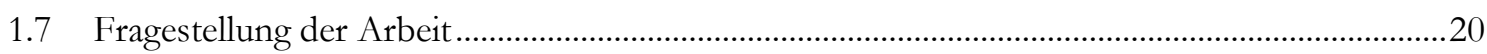

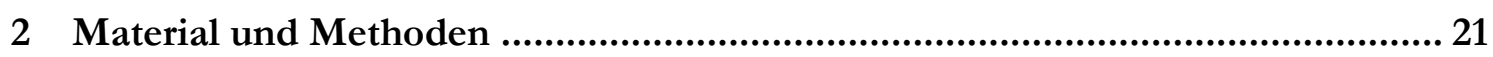

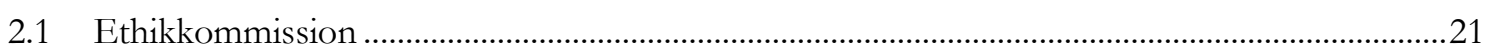

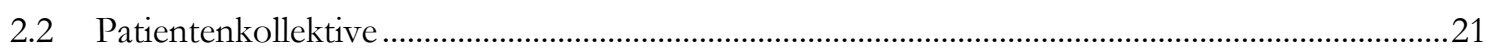

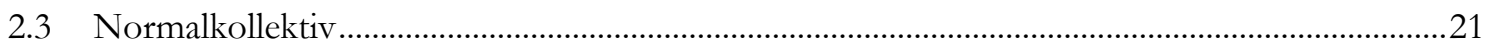

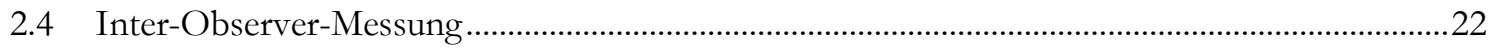

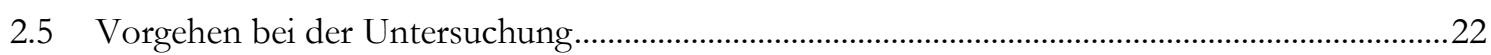

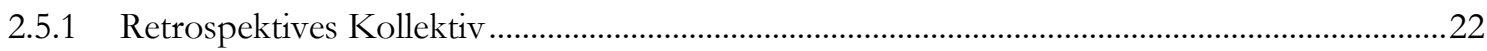

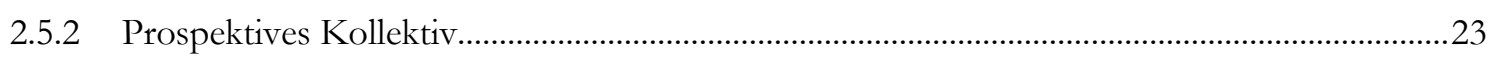

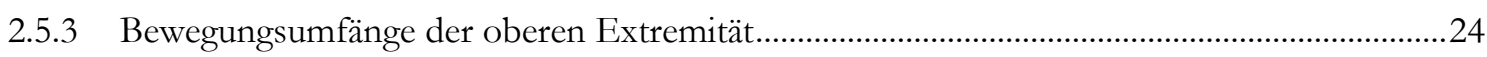

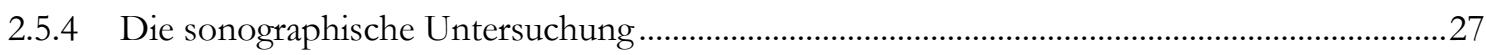

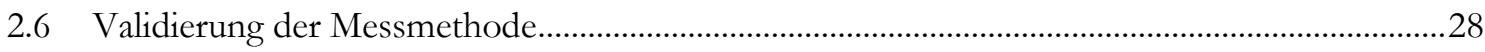




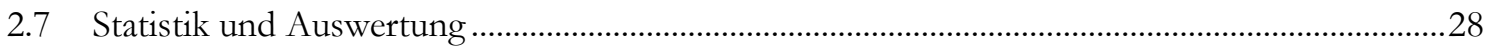

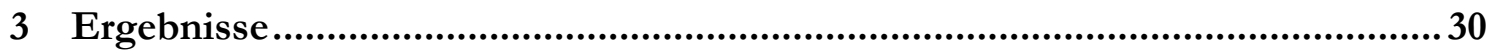

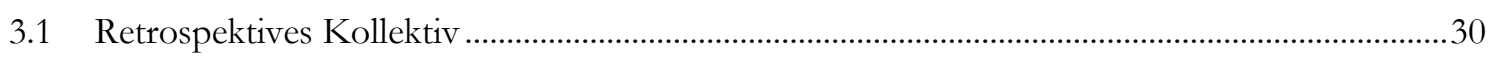

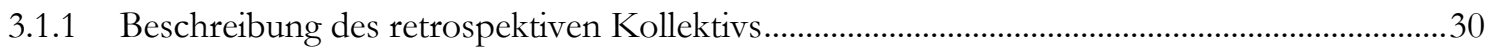

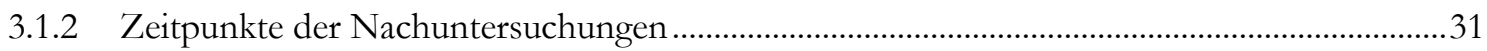

3.1.3 Anamnese und subjektive Beschwerden des retrospektiven Kollektivs .................................32

3.1.4 Klinische Untersuchung des retrospektiven Kollektivs...........................................................33

3.1.4.1 Innenrotation der Schulter im retrospektiven Kollektiv .........................................................33

3.1.4.2 Außenrotation der Schulter im retrospektiven Kollektiv.........................................................36

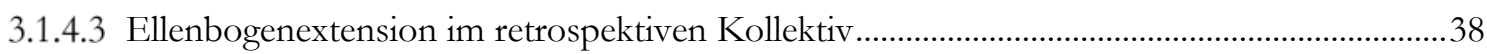

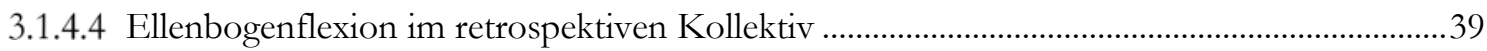

3.1.4.5 Pronation des Unterarmes im retrospektiven Kollektiv ...........................................................41

3.1.4.6 Supination des Unterarmes im retrospektiven Kollektiv .......................................................43

3.1.4.7 Ellenbogenachse des retrospektiven Kollektivs .....................................................................44

3.1.4.8 Sonographische Humerustorsionsmessung im retrospektiven Kollektiv ...............................45

3.1.5 Humerustorsionsänderung im retrospektiven Kollektiv .........................................................49

3.1.5.1 Humerustorsionsdifferenz bei der NU1 in Abhängigkeit von Ausmaß, Richtung und Alter in den Folgeuntersuchungen ..................................................................................... 49

3.1.5.2 Humerustorsionsdifferenz in Korrelation zum Cubitus varus / valgus ..................................52

3.1.5.3 Humerustorsionsdifferenz und Funktion von Schulter/ Ellenbogen ....................................52

3.1.5.4 Humerustorsionsdifferenz im Vergleich zu den Röntgenbildern...........................................56

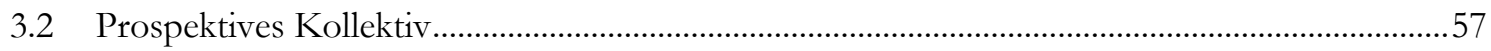

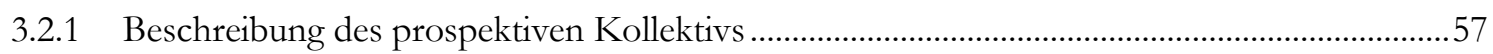

3.2.2 Anamnese und subjektive Beschwerden des prospektiven Kollektivs.....................................59

3.2.3 Klinische Untersuchung des prospektiven Kollektivs .............................................................60

3.2.3.1 Innenrotation der Schulter im prospektiven Kollektiv .............................................................60

3.2.3.2 Außenrotation der Schulter im prospektiven Kollektiv ...........................................................61

3.2.3.3 Ellenbogenextension im prospektiven Kollektiv ..................................................................62

3.2.3.4 Ellenbogenflexion im prospektiven Kollektiv .......................................................................63

3.2.3.5 Pronation und Supination des Unterarmes im prospektiven Kollektiv...................................64

3.2.3.6 Ellenbogenachse des prospektiven Kollektivs......................................................................64

3.2.3.7 Sonographische Humerustorsionsmessung im prospektiven Kollektiv..................................65

3.2.4 Humerustorsionsänderung im prospektiven Kollektiv ...............................................................68

3.2.4.1 Humerustorsionsdifferenz in Abhängigkeit von Ausmaß, Richtung und Alter in den nachfolgenden Untersuchungen .....................................................................................68

3.2.4.2 Humerustorsionsdifferenz in Korrelation zum Cubitus varus / valgus ..................................70

3.2.4.3 Humerustorsionsdifferenz und Funktion von Schulter/ Ellenbogen ....................................71

3.2.4.4 Humerustorsionsveränderung und Analyse der Röntgenbilder................................................73

3.2.4.5 Humerustorsionsdifferenz im Verhältnis zur Händigkeit......................................................74

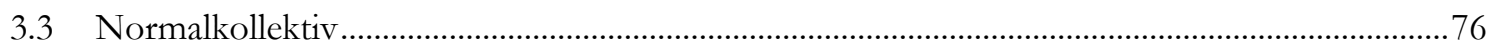

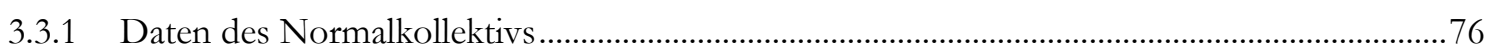

3.3.2 Entwicklung der Humerustorsion im pädiatrischen Normalkollektiv und im

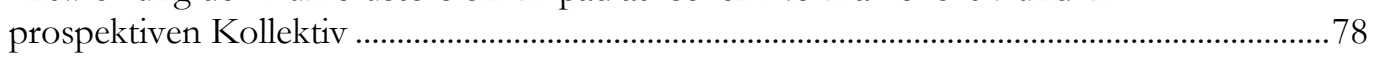

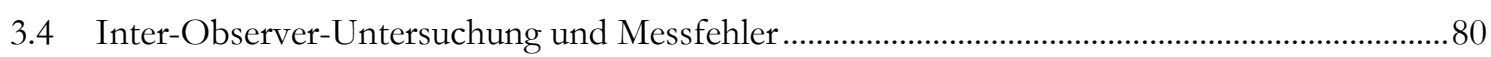

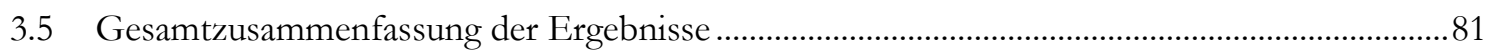




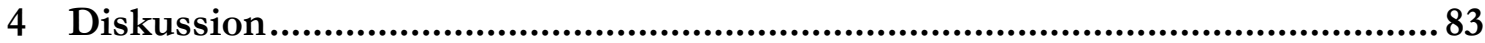

4.1 Problematik der suprakondylären Humerusfraktur im Kindesalter...............................................83

4.2 Konsequenz des posttraumatischen Torsionsfehlers ................................................................83

4.2.1 Bedeutung der posttraumatischen Fehlstellung und der Humerustorsion für Funktion und Ellenbogenachse ................................................................................................................. 85

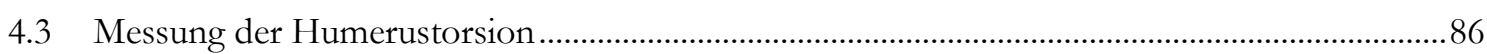

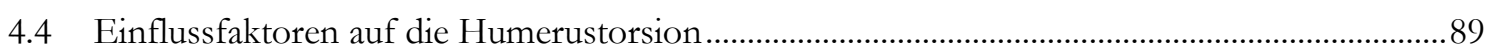

4.5 Korrektur der Torsionsdifferenz nach suprakondylären Humerusfrakturen im

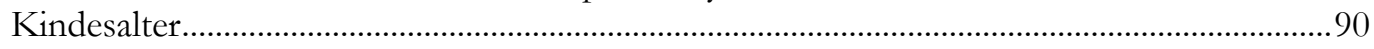

4.6 Fazit, Limitationen der Untersuchung und Ausblick ...................................................................91

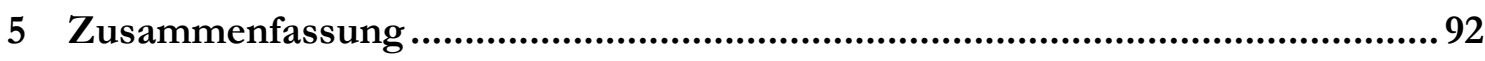

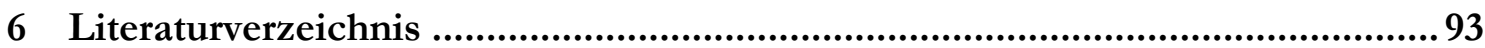




\section{Abbildungsverzeichnis}

Abbildung 1: Rechter Humerus, Ansicht von lateral bzw. medial 3

Abbildung 2: Artikulierende Skelettelemente eines rechten Schultergelenks .................................... 4

Abbildung 3: Artikulierende Skelettelemente eines rechten Ellenbogengelenks................................. 5

Abbildung 4: Einteilung der suprakondylären Humerusfrakturen ........................................................ 7

Abbildung 5: Sensibilitätsbereich von: Nervus medianus, Nervus ulnaris und Nervus radialis ........... 8

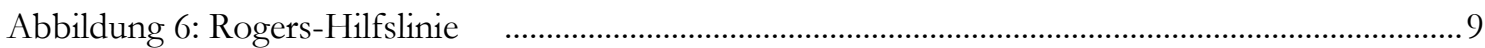

Abbildung 7: Suprakondyläre Humerusfraktur mit Rotationssporn ..................................................10

Abbildung 8: Abhängigkeit der Kontaktfläche der Fraktur von der Lage der Drehachse und dem Ausmaß einer Fehlrotation.............................................................................................12

Abbildung 9: Cubitus varus auf der rechten Seite nach suprakondylärer Humerusfraktur.................13

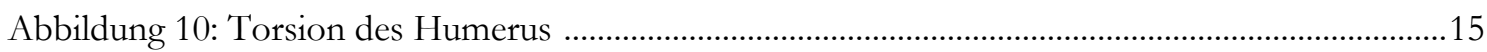

Abbildung 11: Anatomisch-anthropologischer Humerustorsionswinkel..............................................16

Abbildung 12: Beziehung zwischen Antetorsion des Schenkelhalses und Femurkopfkondylenquerachse ........................................................................................19

Abbildung 13: Untersuchungsbogen für das retrospektive und prospektive Kollektiv.....................23

Abbildung 14: Fotodokumentation des Ellenbogenstatus.....................................................................24

Abbildung 15a-g: Klinische Untersuchung und normaler Bewegungsumfang von Schulter-

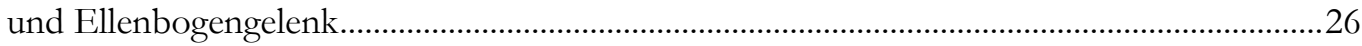

Abbildung 16a-c: Klinische Darstellung der physiologischen Ellenbogenachse, der Cubitusvalgus- und varus-Stellung................................................................................................26

Abbildung 17: Materialien für die Untersuchung: Lagerungsschale, Linearschallkopf mit montierter Wasserwaage und Lagerungsschale in Verwendung

Abbildung 18: Darstellung des Sulcus intertubercularis am Humeruskopf .........................................28

Abbildung 19: Altersverteilung des retrospektiven Kollektivs (N=22) zum Unfallzeitpunkt ............30

Abbildung 20: Dislozierte suprakondyläre Humerusfraktur bei einem 9-Jährigen Kind; Operative Versorgung der Fraktur mittels eines radialen Fixateur externe .........................31

Abbildung 21: Altersverteilung des prospektiven Kollektivs zum Unfallzeitpunkt ..........................58

Abbildung 22: Perkutane K-Draht-Osteosynthese von radial und ulnar..........................................59

Abbildung 23: Altersverteilung des Kinder-Normalkollektivs .........................................................77

Abbildung 24: Verlauf der sonographisch gemessenen Humerustorsion mit zunehmenden Alter im Kinder-Normalkollektiv

Abbildung 25: Humerustorsion der frakturierten und gesunden oberen Extremität im prospektiven Kollektiv von Kindern mit vorhandener Humerustorsionsdifferenz im Altersverlauf. 


\section{Tabellenverzeichnis}

Tabelle 1: Verschiedene Torsionswerte in der Literatur

Tabelle 2: Vergleich der verschiedenen Humerustorsionsmessmethoden in Bezug auf deren Korrelation

Tabelle 3: Testbedingungen und ihre dazugehörigen Testverfahren

Tabelle 4: Unfallhergang und Verteilung der Unfallmuster der Kinder des retrospektiven Kollektivs

Tabelle 5: Subjektive Beschwerden des retrospektiven Kollektivs zu den jeweiligen

Untersuchungsterminen

Tabelle 6: Ergebnisse der Untersuchung der Schulterinnenrotation in den

Nachuntersuchungen im retrospektiven Kollektiv 35

Tabelle 7: Ergebnisse der Untersuchung der Schulteraußenrotationen in den

Nachuntersuchungen im retrospektiven Kollektiv

Tabelle 8: Ergebnisse der Untersuchung der Ellenbogenextension bei den jeweiligen

Nachuntersuchungen im retrospektiven Kollektiv

Tabelle 9: Ergebnisse der Untersuchung der Ellenbogenflexion bei den jeweiligen

Nachuntersuchungen im retrospektiven Kollektiv

Tabelle 10: Ergebnisse der Untersuchung der Pronation bei den jeweiligen

Nachuntersuchungen im retrospektiven Kollektiv

Tabelle 11: Ergebnisse der Untersuchung der Supination bei den jeweiligen

Nachuntersuchungen im retrospektiven Kollektiv

Tabelle 12: Ergebnisse der Untersuchung der Achsmessung bei den jeweiligen

Nachuntersuchungen im retrospektiven Kollektiv ....

Tabelle 13: Ergebnisse der Untersuchung der sonographisch gemessenen

Humerustorsionswerte im retrospektiven Kollektiv....

Tabelle 14: Ergebnisse der Untersuchung der sonographisch gemessenen

Humerustorsionsdifferenz im retrospektiven Kollektiv

Tabelle 15: Ergebnisse der Untersuchung der Humerustorsionsdifferenzänderung in

Abhängigkeit vom Ausmaß der primären Torsionsdifferenz und vom Alter bei der NU1

Tabelle 16: Ergebnisse der Untersuchung der Torsionsdifferenz in allen Nachuntersuchungen in Abhängigkeit vom Alter bei der 1. NU.

Tabelle 17: Ergebnisse der Untersuchung der Humerustorsionsdifferenz bei der NU1 im Vergleich zur Differenz der Ellenbogenachse

Tabelle 18: Ergebnisse der Untersuchung der Humerustorsionsdifferenz im Verhältnis zur

Differenz der Schulteraußenrotation in den Nachuntersuchungen 1-3

Tabelle 19: Ergebnisse der Untersuchung der Humerustorsionsdifferenz bei der NU1 im Verhältnis zur Differenz der Schulterinnenrotation in den Nachuntersuchungen 1-3 .........54

Tabelle 20: Ergebnisse der Untersuchung der Humerustorsionsdifferenz bei der NU1 im Vergleich zur Differenz der Ellenbogenextension in den Nachuntersuchungen..................55

Tabelle 21: Ergebnisse der Untersuchung der Humerustorsionsdifferenz bei der NU1 im Vergleich zur Differenz der Ellenbogenflexion in den Nachuntersuchungen

Tabelle 22: Ergebnisse der Untersuchung der Humerustorsionsdifferenz im Verhältnis zu den Röntgenaufnahmen

Tabelle 23: Unfallhergang und Verteilung der Unfallmuster der Kinder des prospektiven Kollektivs

Tabelle 24: subjektive Beschwerden im prospektiven Kollektiv zu den jeweiligen

Nachuntersuchungsterminen

Tabelle 25: Ergebnisse der Untersuchung der Schulterinnenrotationen im prospektiven Kollektiv. 
Tabelle 26: Ergebnisse der Untersuchung der Schulteraußenrotation im prospektiven Kollektiv ....62

Tabelle 27: Ergebnisse der Untersuchung der Ellenbogenextension im prospektiven Kollektiv ......63

Tabelle 28: Ergebnisse der Untersuchung der Ellenbogenflexion im prospektiven Kollektiv............64

Tabelle 29: Ergebnisse der Untersuchung der Mittelwerte der absoluten klinischen Achsmessung am Arm im prospektiven Kollektiv.

Tabelle 30: Ergebnisse der Untersuchung der sonographisch gemessenen Humerustorsionswerte bei Kindern im prospektiven Kollektiv

Tabelle 31: Ergebnisse der Untersuchung der sonographisch gemessenen Humerustorsionsdifferenz bei Kindern im prospektiven Kollektiv

Tabelle 32: Ergebnisse der Untersuchung der sonographisch gemessenen

Humerustorsionswerte bei Kindern mit sonographisch gemessenen Differenzen zwischen beiden Extremitäten

Tabelle 33: Humerustorsionsdifferenzänderung in Abhängigkeit vom Ausmaß der primären

Torsionsdifferenz bei der NU nach 6 Wochen und in Abhängigkeit vom Alter 6 Wochen nach Unfall.

Tabelle 34: Torsionsdifferenz bei allen Nachuntersuchungszeitpunkten in Abhängigkeit vom Alter

Tabelle 35: Humerustorsionsdifferenz bei der NU nach vier Monaten im Vergleich zur Ellenbogenachsendifferenz zwischen dem gesunden und frakturierten Arm in den folgenden Nachuntersuchungen.

Tabelle 36: Ergebnisse der Untersuchung der Humerustorsionsdifferenz im Verhältnis zur Schulteraußenrotationsdifferenz in den nachfolgenden Nachuntersuchungen

Tabelle 37: Ergebnisse der Untersuchung der Humerustorsionsdifferenz nach 6 Wochen im Verhältnis zur Schulterinnenrotationsdifferenz in den Nachuntersuchungen..

Tabelle 38: Ergebnisse der Untersuchung der Humerustorsionsdifferenz nach 6 Wochen im Vergleich zur Ellenbogenextensionsdifferenz in den Nachuntersuchungen...

Tabelle 39: Ergebnisse der Untersuchung der Humerustorsionsdifferenz nach 6 Wochen im Vergleich zur Ellenbogenflexionsdifferenz in den Nachuntersuchungen

Tabelle 40: Ergebnisse der Untersuchung der Humerustorsionsdifferenz nach 6 Wochen im Vergleich zu den Röntgenaufnahmen mit radiologischen Auffälligkeiten

Tabelle 41: Humerustorsionsdifferenz ( $\mathrm{M} \pm 1 \mathrm{SD})$ in Abhängigkeit der Händigkeit............................75

Tabelle 42: Mittelwert ( \pm 1 Standardabweichung) der Humerustorsion in den Normalkollektiven.

Tabelle 43: Durchschnittliches Alter der Kinderkollektive...

Tabelle 44: Gesamte sonographisch gemessene Humerustorsion im Kinder-Normalkollektiv in unterschiedlichen Altersgruppen.

Tabelle 45: Durchschnittliche Humerustorsion des Normalkollektivs aufgeteilt in drei Altersgruppen

Tabelle 46: Mittelwerte ( \pm 1 Standardabweichung) der sonographisch gemessenen Humerustorsionen der jeweiligen Untersucher

Tabelle 47: Übersicht über die einzelnen OP-Verfahren und deren

Funktionseinschränkungen/ Achsfehlstellungen 


\section{Abkürzungsverzeichnis}

$\begin{array}{ll}\text { ESIN } & \text { elastic stable intramedullary nailing } \\ \text { NU } & \text { Nachuntersuchung } \\ \text { AWMF } & \begin{array}{l}\text { Arbeitsgemeinschaft der Wissenschaftlichen Medizinischen Fachgesell- } \\ \text { schaften e.V. }\end{array} \\ \text { a.p. } & \text { anterior/ posterior } \\ \text { AO } & \text { Arbeitsgemeinschaft für Osteosynthesefragen e.V. } \\ \text { AHL } & \text { anterior humeral line } \\ \text { AL } & \text { anteriorly loss } \\ \text { A } & \text { anterior third } \\ \text { P } & \text { posterior third } \\ \text { M } & \text { middle third } \\ \text { PL } & \text { posteriorly loss } \\ \text { MRT } & \text { Magnetresonanztomographie } \\ \text { CT } & \text { Computertomographie } \\ \text { OP } & \text { Operation } \\ \text { p- Wert } & \text { Ergebnis eines statistischen Signifikanztest } \\ \text { K-Draht } & \text { Kirschnerdraht } \\ \text { N } & \text { Nervus }\end{array}$




\section{$1 \quad$ Einleitung}

\subsection{Einführung}

Dislozierte Ellenbogenfrakturen im Kindesalter sind seit dem Altertum bekannt (Scharizer 1964). Eine genaue Klassifizierung gelang aber erst mit Einführung der Röntgendiagnostik (Scharizer 1964). Im Lehrbuch „Knochenbrüche bei Kindern“ (1957) von Blount wurde erstmals eine differenzierte Klassifizierung und Analyse von Ellenbogenfrakturen beschrieben. Er bemerkte, dass Ellenbogenfrakturen bei Kindern im Alter von drei bis zehn Jahren, mit einem Altersgipfel zwischen fünf und acht Jahren, am häufigsten vorkommen. Die suprakondyläre Humerusfraktur kommt mit ca. 4 -7\% aller Frakturen im Kindesalter vor (Fernandez und Eberhardt 2012; Gercek et al. 2015; Hefti 2015) und stellt mit 80\% die häufigste Fraktur im Bereich des Ellenbogens dar (Hefti 2015).

Die suprakondyläre Humerusfraktur ist wegen ihrer zahlreichen Komplikationsmöglichkeiten, wobei die Bildung eines Cubitus varus zu den häufigsten zählt, problematisch (French 1959, Hefti 2015, Davis et al. 2000, Kallio et al. 1992, Labelle et al. 1982, Strohm 2012, von Laer 1979). Ein Cubitus varus am Ellenbogen ist mit einem posttraumatischen Torsionsfehler am Humerus assoziiert (Harland et al. 1991, Kunz et al. 1993, Dähnert und Bernd 1986, Söderlund et al. 1989), wobei klinische, radiologische und sonographische Verfahren zur Diagnosestellung oft ungenau sind (Dähnert und Bernd 1986; Fuchs et al. 1991; Harland et al. 1991; Kunz et al. 1993). Nach von Laer et al. (2013) entsteht ein Torsionsfehler nach suprakondylärer Humerusfraktur im Kindesalter durch eine Torsion der Frakturfragmente zueinander. Dies ist laut dem Autor (von Laer et al. 2013) als ventraler Sporn zu tasten und im Röntgenbild sichtbar. Der Sporn kann sich mit zunehmendem zeitlichen Abstand zur Fraktur remodellieren, der Torsionsfehler bleibt jedoch bestehen (von Laer et al. 2013). Eine genaue Klassifizierung und Diagnostik sowie die Entwicklung des Humerustorsionsfehlers während des verbleibenden Wachstums wurde bisher noch nicht systematisch untersucht. Um ein entsprechendes Remodelling nachzuweisen bzw. um die Grenzen der Spontankorrektur aufzuzeigen, wurde in der vorliegenden Studie jeweils in einem retrospektiven- und prospektiven Kollektiv von Kindern mit operativ versorgten suprakondylären Humerusfrakturen der Langzeitverlauf analysiert. Da eine standardisierte Diagnostikmethode nicht vorhanden ist, wurde eine sonographisch durchzuführende Messung zur Humerustorsion beschrieben, an einem gesunden Kollektiv validiert und im Verlauf analysiert. 


\subsection{Die obere Extremität}

\subsubsection{Anatomie des Humerus sowie des Schulter- und Ellenbogengelenkes}

Die Anatomie des Oberarmes (lateinisch Humerus) ist in allen gängigen Anatomiebüchern nachzulesen. Laut dem Anatomiebuch von Steinbrück et al. (2008) besteht die obere Extremität aus vier Abschnitten: Schultergürtel, Oberarm, Unterarm und Hand.

In den beiden Anatomielehrbüchern von Steinbrück et al. (2008) und Zilles und Tillmann (2010) ist der Humerus ein langer Röhrenknochen. Dieser wird in den Schaft (Diaphyse) und in zwei Endabschnitte (Epiphysen) unterteilt. Die proximale Epiphyse ist der Kopf (Caput humeri) des Oberarmes.

Laut Aumüller et al. (2014) schließt sich an das Caput humeri das Collum humeri (Hals) an. Hier gibt es eine kleine Einziehung, das Collum anatomicum. Diese dient der Abgrenzung vom Kopf gegen das Corpus und zwei Apophysen (Tuberculum majus und minus). Das Tuberculum majus ist nach lateral und das Tuberculum minus nach ventral gerichtet. Hier setzen die Muskeln der „Rotatorenmanschette“ an. Zwischen den beiden Knochenvorsprüngen ist der Sulcus intertubercularis. Im Sulcus verläuft die Sehne des Caput longum des Musculus biceps brachii. Von den Tubercula aus weiter distal finden sich die Crista tuberculi majoris bzw. minoris. Zwischen dem Humeruskopf und der proximalen Metaphyse befindet sich das Collum chirurgicum. Hier können viele Frakturen entstehen. Weiterhin gibt es am Humerus den Corpus humeri. Hier gibt es die Tuberositas deltoidea, welche eine Ansatzstelle für den Musculus deltoideus ist. Von dort verläuft distal und dorsal der Sulcus nervi radialis. Der Aufbau des Humerus ist in der Abbildung 1 dargestellt.

Der Humeruskopf bildet zusammen mit dem Humerusschaft einen Winkel von $135^{\circ}$. Im Bereich des distalen Humerus existiert die Trochlea humeri (medial) und das Capitulum humeri (lateral), welche als Gelenkflächen den proximalen Teil des Ellenbogengelenkes bilden (Aumüller et al. 2014).

Als Retrotorsionswinkel bzw. Humerustorsionswinkel wird die Achse durch den Humeruskopf- und hals bezeichnet, die sie mit der Achse durch die Epikondylen bildet. Dieser beträgt $16^{\circ}$ (Zilles und Tillmann 2010; Aumüller et al. 2014). 


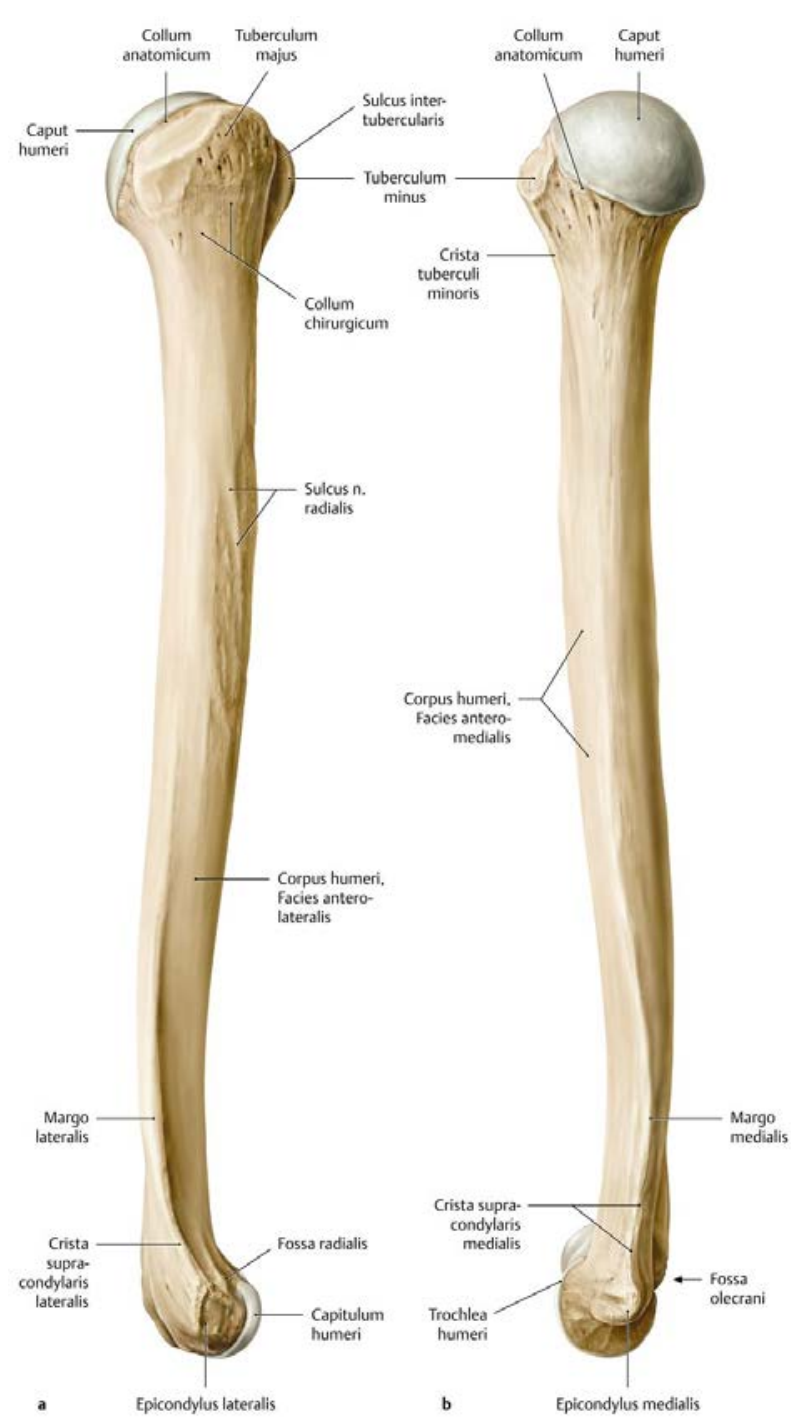

Abbildung 1: Rechter Humerus; a: Ansicht von lateral; b: Ansicht von medial (Schünke et al. 2011). Der Abdruck erfolgt mit freundlicher Genehmigung des Thieme-Verlags

Das Schultergelenk (Abbildung 2) ist ein Kugelgelenk mit drei Freiheitsgraden (Aumüller et al. 2014). Die Gelenkpfanne besteht aus der Cavitas glenoidalis der Scapula. Caput humeri und Cavitas glenoidalis berühren sich nur zu 1/3 bis 1/4. Das Labrum glenoidale (Gelenklippe) vertieft die Pfanne des Schultergelenkes und vergrößert die Kontaktfläche zwischen Humeruskopf und Cavitas glenoidalis (Aumüller et al. 2014). An der Außenseite der Gelenklippe ist die Kapsel angeheftet. Die Kapsel wird durch verschiedene Bänder verstärkt: Ligamentum glenohumeralia superius-, medium und -inferius (anteriorer Teil) sowie Ligamentum coracohumerale. Erstere werden bei einer Außenrotation angespannt. Letzteres hemmt die Außenrotation bei adduziertem Arm. Das sogenannte „Dach des Schultergelenks" wird aus dem Ligamentum coracoacromiale, dem Acromium und dem Processus coracoideus gebildet. Somit wird eine kraniale Luxation des Humeruskopfes erschwert (Aumüller et al. 2014). 


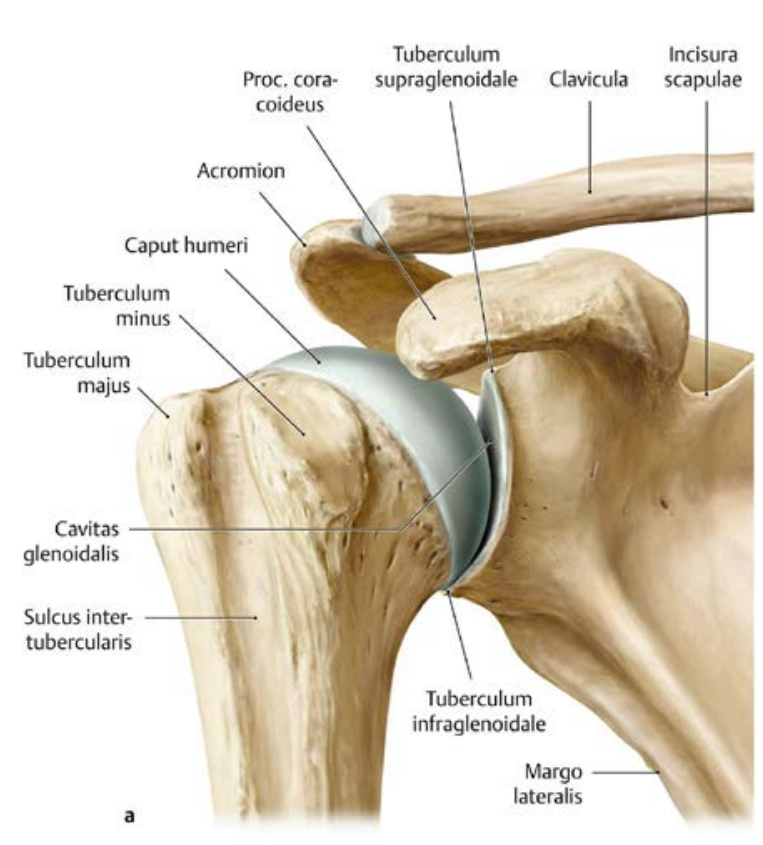

Abbildung 2: Artikulierende Skelettelemente eines rechten Schultergelenks (Articulatio humeri). Ansicht von ventral (Schünke et al. 2011). Der Abdruck erfolgt mit freundlicher Genehmigung des Thieme-Verlags

Das Ellenbogengelenk (Articulatio cubiti, Abbildung 3) ist ein zusammengesetztes Gelenk (Articulatio composita), da folgende Knochen daran beteiligt sind: die distale Epiphyse des Humerus, die proximale Epiphyse des Radius (Speiche) und die Ulna (Elle) (Aumüller et al. 2014). Das Ellenbogengelenk ist aufgrund seiner Kombination eines Scharniergelenkes mit einer Rotationsmöglichkeit um die Längsachse ein Dreh-ScharnierGelenk (Steinbrück et al. 2008). Beim Articulatio humeroulnaris handelt es sich um ein Schaniergelenk. Somit sind Extension und Flexion möglich. Das Articulatio humeroradialis ist strukturell ein Kugelgelenk, ist aber durch die Fixierung des Radius an der Ulna ebenfalls auf Extension und Flexion beschränkt. Das proximale Radioulnargelenk ist ein Rad- oder Zapfengelenk. Zusammen mit dem distalen Radioulnargelenk wird die Auswärts- und Einwärtsbewegung (Supination bzw. Pronation) ermöglicht (Steinbrück et al. 2008). 


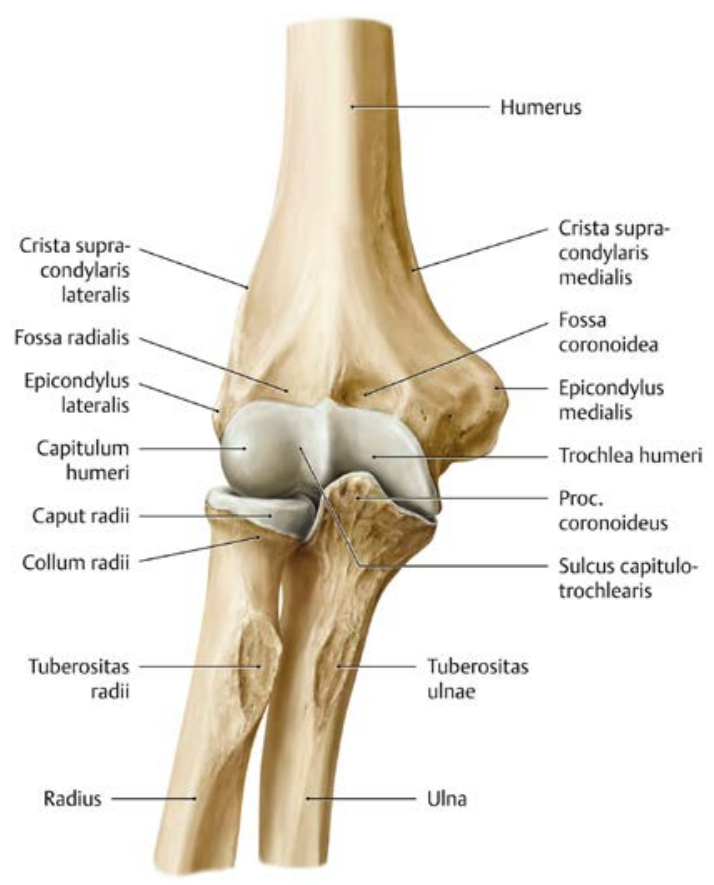

Abbildung 3: Artikulierende Skelettelemente eines rechten Ellenbogengelenks (Schünke et al. 2011). Der Abdruck erfolgt mit freundlicher Genehmigung des Thieme-Verlags

\subsection{Die suprakondyläre Humerusfraktur}

Die suprakondyläre Humerusfraktur macht ca. $4-7 \%$ aller Frakturen im Kindesalter (Weinberg et al. 2002; Schofer et al. 2003; Fernandez und Eberhardt 2012; Gercek et al. 2015; Hefti 2015) und im Bereich des Ellenbogens 80\% (Hefti 2015) aller ellenbogennahen Frakturen aus. Der Häufigkeitsgipfel liegt um das fünfte bis siebte Lebensjahr (Lubinus 1924; Schofer et al. 2003; Kraus et al. 2005; Wessel 2010; Fernandez und Eberhardt 2012; Hefti 2015).

\subsubsection{Pathophysiologie der suprakondylären Humerusfrakturen}

Die Ursache sind oft Stürze beim Sport oder in der Freizeit. Es wird dabei der Extensionsund Flexionstyp unterschieden (Fernandez und Eberhardt 2012).

Nach den beiden Autoren (Fernandez und Eberhardt 2012) kommt es zum Extensionstyp (bei 98\% aller suprakondylären Humerusfrakturen), wenn ein indirektes Trauma, z. B. „Sturz auf den ausgestreckten Arm“ resultiert. Zu einem Flexionstyp (bei 2\% aller suprakondylären Humerusfrakturen) kommt es, wenn die Kinder direkt auf das Ellenbogengelenk stürzen. 


\subsubsection{Klassifikation der suprakondylären Humerusfrakturen}

Um die suprakondylären Humerusfrakturen einzuteilen, gab es in der Vergangenheit mehrere Klassifikationen (Lutz et al. 2011; Slongo und Audigé 2007; Fernandez und Eberhardt 2012; Leitlinie suprakonyläre Humerusfraktur beim Kind 2014). Zunächst wurde die Fraktur in einen Flexions- und Extensionstyp eingeteilt (Fernandez und Eberhardt 2012). Weitere Einteilungen kamen von Baumann, Lubinus und Felsenreich (Lubinus 1924; Felsenreich 1931; Baumann 1960; Weinberg et al. 2002; Schofer et al. 2003). Im anglo-amerikanischen Raum wird die Gartland-Klassifikation am häufigsten benutzt (Schofer et al. 2003; Fernandez und Eberhardt 2012). Hierbei erfolgte die Einteilung in drei Grade: Gartland I: keine Dislokation, Gartland II: Dislokation in einer Ebene und Gartland III: komplett disloziert (Gartland 1959). Aber auch die Einteilung nach Holmberg wird verwendet (Holmberg 1945; Weinberg et al. 2002; Corres-Franco und Torres-Gómez 2015). Doch diese Einteilungen berücksichtigen nicht die Rotation der Fragmente, welches als ein Zeichen von Instabilität gilt (Hasler 2001).

In Deutschland und in anderen deutschsprachigen Ländern werden die Frakturen der langen Röhrenknochen im Kindesalter nach der Arbeitsgemeinschaft für Osteosynthesefragen (AO) in Anlehnung an die Klassifikation nach von Laer eingeteilt (von Laer et al. 2002; Slongo und Audigé 2007; Lutz et al. 2011; von Laer et al. 2013; Leitlinie supra-kondyläre Humerusfraktur beim Kind 2014; Fernandez et al. 2016), die die Rotation der Fragmente berücksichtigt. Hierbei werden vier Typen unterschieden (von Laer et al. 2013; Abbildung 4):

Typ I: keine Dislokation (stabil)

Typ II: Dislokation in einer Ebene (Ante- oder Rekurvation; stabil und drohend instabil) Typ III: Dislokation in zwei Ebenen (Ante- oder Rekurvation, Rotationsfehler oder Seitzu-Seit-Verschiebung in der Frontalebene)

Typ IV: Dislokation in drei Ebenen oder vollständige Dislokation ohne knöchernen Kontakt (Ante- oder Rekurvation, Rotationsfehler und Seit-zu-Seit-Verschiebung) 


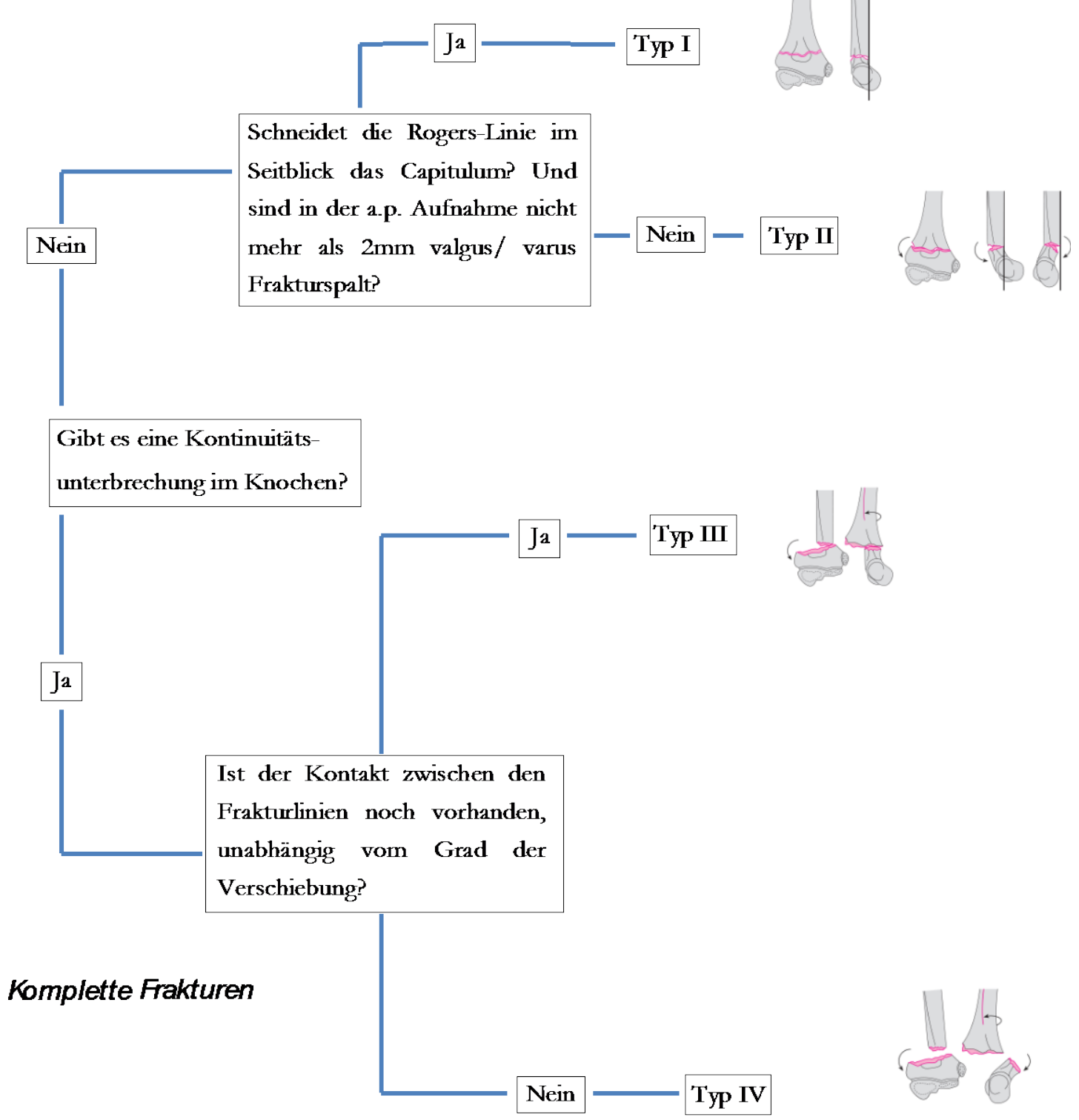

Abbildung 4: Einteilung der suprakondylären Humerusfrakturen. a.p. $=$ anterior und posterior (modifiziert nach Leitlinie suprakondyläre Humerusfraktur beim Kind 2014; Bilder Baierlein 2011). Der Abdruck erfolgt mit freundlicher Genehmigung des ThiemeVerlags

\subsubsection{Diagnostik der suprakondylären Humerusfrakturen}

Die Diagnostik der suprakondylären Humerusfraktur im Wachstumsalter erfolgt anhand der Anamnese sowie der klinischen und radiologischen Untersuchung.

Bei der Anamneseerhebung steht die Frage nach dem Traumaereignis im Vordergrund. Ziel sollte es sein, die Lokalisation der möglichen Läsion und das subjektive Ausmaß der Schmerzen zu eruieren (von Laer et al. 2013). 
Die klinische Untersuchung sollte zunächst visuell erfolgen. So können schon Schwellugen, Fehlstellungen, Deformierungen usw. festgestellt werden. Dann sollte der Arm hinsichtlich Durchblutung, Motorik und Sensibilität untersucht werden (von Laer et al. 2013). Der Nervus (N.) medianus, N. radialis und N. ulnaris sollten überprüft werden (Leitlinie suprakondyläre Humerusfraktur beim Kind 2014, Abbildung 5). Hinsichtlich der Durchblutung wird geraten, die Arteria radialis und Arteria ulnaris zu tasten (Leitlinie suprakondyläre Humerusfraktur beim Kind 2014). Von Laer et al. (2013) empfahl, die klinisch-manuelle Diagnostik einer Fraktur oder Luxation nicht durchzuführen, da hierdurch für das Kind starke Schmerzen entstehen würden.

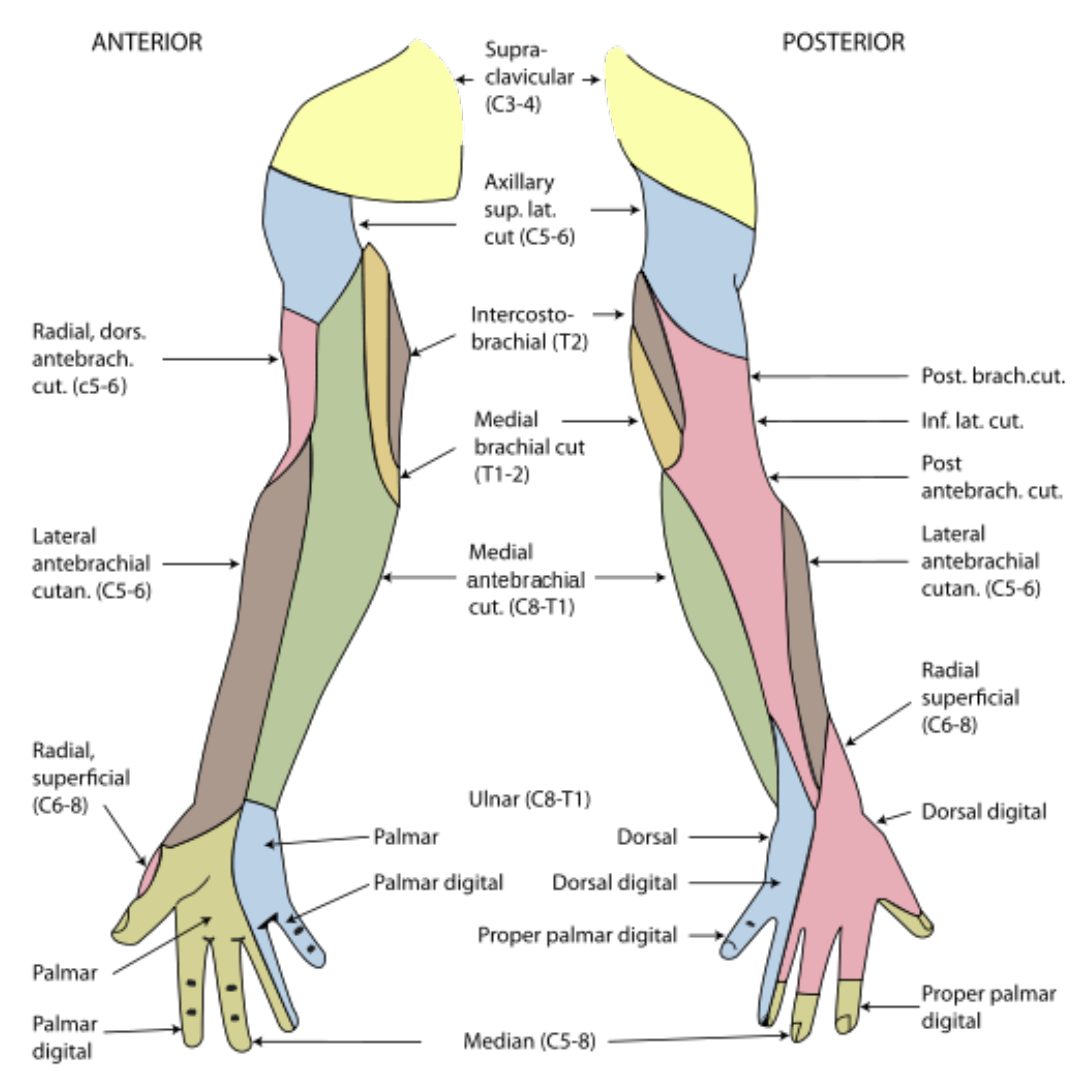

Abbildung 5: Sensibilitätsbereich von: Nervus medianus (hellgrün), Nervus ulnaris (blau) und Nervus radialis (rosa) (Gray 1918). Lizenziert durch eine Creative-Commons-Lizenz

Um den Verdacht einer Humerusfraktur zu erhärten, muss eine Röntgenuntersuchung des Ellenbogens in zwei Ebenen (von a.p. und seitlich) erfolgen (Kraus et al. 2007). Diese dient der Diagnosestellung und der Planung der korrekten Therapie. So können z. B. „KadiLäsionen“ (von Laer et al. 2013) vermieden werden. Diese sind als schwerwiegende Komplikationen bei unscheinbar erscheinenden Verletzungen definiert, die bei Übersehen zu Funktionseinschränkungen und Deformitäten führen und damit häufig einen Haftpflichtprozess nach sich ziehen (von Laer et al. 2013). Eine Röntgenaufnahme der Gegenseite, also des nicht frakturierten Armes, wird heutzutage nicht mehr empfohlen (Kraus et al. 2007; von Laer et al. 2013; Sommerfeldt 2013). Besteht ein Torsionsfehler bei 
suprakondylären Humerusfrakturen, so kann es zum Abkippen des distalen Fragments nach ulnar und zu einer Varusdeformität kommen (von Laer et al. 2013). In der Röntgenaufnahme ist der Torsionsfehler meist an einem ventralen, seltener an einem dorsalen Sporn zu erkennen (von Laer et al. 2013). Zur Unterscheidung, ob es sich um einen Extensions- oder Flexionsbruch handelt, wird im Röntgen die Rogers-Hilfslinie eingezeichnet (Rogers et al. 1978; von Laer et al. 2013).
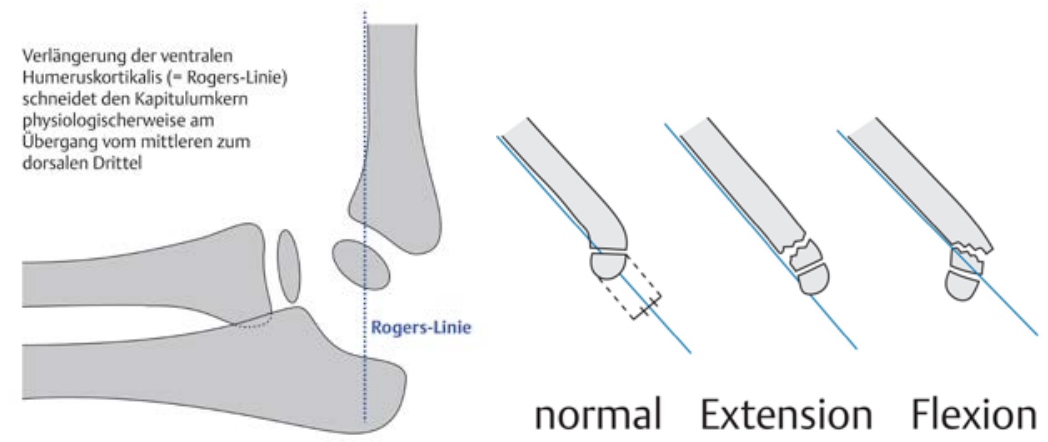

normal Extension Flexion

Abbildung 6: Rogers-Hilfslinie (links Jorden 2014, rechts von Laer et al. 2013). Der Abdruck erfolgt mit freundlicher Genehmigung des Thieme-Verlags

Diese Linie wird im englischen anterior humeral line (AHL) genannt und geht entlang des vorderen distalen Humerus und schneidet dann das Capitulum humeri in einem bestimmten Abschnitt (Kao et al. 2016). Diese Abschnitte werden laut Kao et al. (2016) unterteilt in einen vorderen Abschnitt ohne Verbindung (im englischen abgekürzt als AL = anteriorly loss), in ein vorderes Drittel (englisch anterior third $=\mathrm{A}$ ), in ein mittleres Drittel (englisch middle third $=\mathrm{M}$ ), in ein hinteres Drittel (englisch posterior third $=\mathrm{P}$ ) und in einen hinteren Abschnitt ohne Verbindung (englisch posteriorly loss = PL). Der Autor Kao et al. (2016) postuliert, dass wenn die AHL das Capitulum humeri im mittleren oder hinteren Drittel kreuzt, dass dann die Kinder früher die Möglichkeit hätten, eine Flexion im Ellenbogen durchzuführen, als die Kinder, wo die AHL das vordere Drittel schneidet.

Das Capitulum humeri hat eine physiologische Kippung gegenüber dem Schaft von

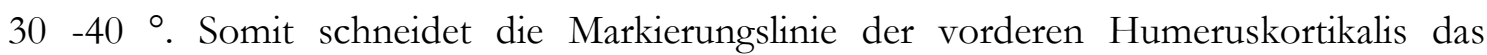
Capitulum am Übergang vom mittleren zum hinteren Drittel. Bei den Extensionsfrakturen (Antekurvation) würde dann die Linie im vorderen Bereich des Capitulum oder noch davor liegen. Bei den Flexionsfrakturen (Rekurvation) schneidet die Linie das hintere Drittel oder liegt hinter dem Capitulum (Rogers et al. 1978; von Laer et al. 2013).

Wichtig ist auch, dass der Rotationssporn (Abbildung 7) nicht mit einer Seit-zu-SeitVerschiebung verwechselt wird (von Laer et al. 2013). In der seitlichen Röntgenaufnahme kann auch zusätzlich durch die Kontur der Fossa olecrani beurteilt werden, ob eine Fehlrotation vorliegt oder nicht (von Laer et al. 2013). Ein Torsionsfehler bis zu $20^{\circ} \mathrm{kann}$ übersehen werden, da er sich hinter dem radialen Kondylus verstecken kann (von Laer et al. 2013). 


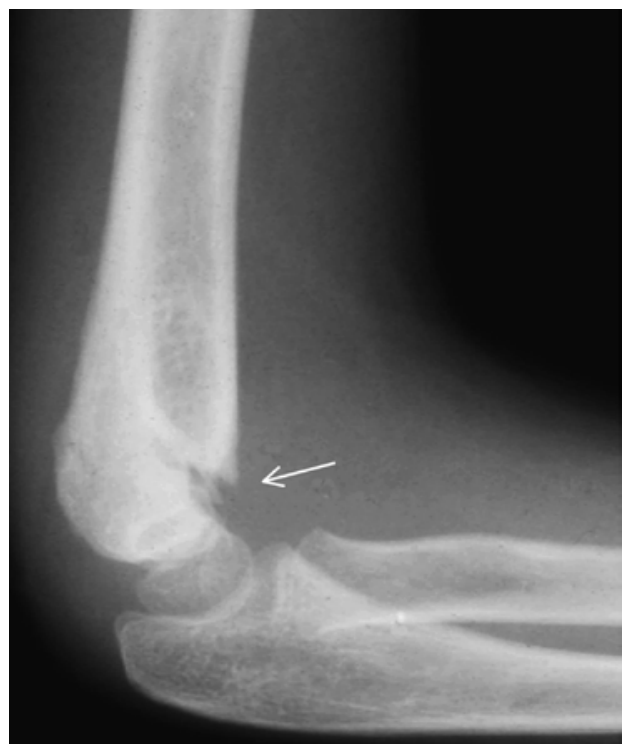

Abbildung 7: Suprakondyläre Humerusfraktur mit Rotationssporn (Benz-Bohm 2005). Der Abdruck erfolgt mit freundlicher Genehmigung des Thieme-Verlags

\subsubsection{Therapie der suprakondylären Humerusfraktur}

In der Literatur (von Laer et al. 2013; Leitlinie suprakondyläre Humerusfraktur beim Kind 2014; Gercek et al. 2015) wird empfohlen die Typ-I-Frakturen konservativ zu behandeln. Dies kann mittels einer Oberarmgipsschiene erfolgen. Hierbei sollte der Unterarm in $90^{\circ}$ Ellenbogenflexion in Neutralstellung ruhiggestellt werden (Gercek et al. 2015). Alternativ kann auch ein Cuff- and Collar-Verband/ Blount Schlinge angelegt werden. Hier wird das Ellenbogengelenk in ca. $110^{\circ}$ Flexion mit gleichzeitiger Pronation fixiert (Gercek et al. 2015).

Typ-II-Frakturen werden, wenn sie stabil sind und nur eine mäßige Antekurvationsstellung haben, in der Blount-Schlinge behandelt, d. h. in die Spitzwinkelstellung gebracht und Frakturen, die leicht rekurviert sind, sollten bei möglichst gestrecktem Ellenbogen ruhig gestellt werden (von Laer et al. 2013). Sind die Typ-IIFrakturen drohend instabil mit größerer Ante- oder Rekurvation $\left(>20^{\circ}\right)$ sollte ebenfalls die Spitzwinkelstellung vorgenommen werden. Falls eine Schwellung im Ellenbogenbereich vorliegt, muss der Ellenbogen nach Abschwellung (ca. zwei bis vier Tage nach dem Unfall) unter Schmermittelgabe in die Spitzwinkelstellung gebracht werden. Dabei kann es zu einem Durchbrechen des Kondylenpfeilers (meist des ulnaren) kommen (von Laer et al. 2013). Ein seitliches Röntgenbild am vierten Tag nach Unfall sollte deshalb durchgeführt werden (von Laer et al. 2013). Kommt es bei den instabilen Typ-II-Frakturen zum sofortigen Repositionsverlust, sollte operativ behandelt werden (Gercek et al. 2015). Ebenfalls wenn ein sekundärer Rotationsfehler auftritt (von Laer et al. 2013). 
Handelt es sich jedoch um eine Typ-II-Flexionsfraktur, sollte eine Blount-Schlinge wegen der Unterstützung der Fehlstellung nicht verwendet werden. Hier ist dann meist eine Operation indiziert (Gercek et al. 2015).

Operationen sind bei instabilen Typ-II- sowie Typ-III und Typ-IV-Frakturen indiziert (Leitlinie suprakondyläre Humerusfraktur beim Kind 2014; Gercek et al. 2015).

Die Frakturtypen III und IV (aber auch die instabile Typ-II Fraktur) sollten in einer Notfalloperation in Narkose zunächst geschlossen reponiert werden (von Laer et al. 2013). Nach den Angaben in der Literatur (Kumar et al. 2002; Blakey et al. 2009; Pretell-Mazzini et al. 2010; White et al. 2010; Leitlinie suprakondyläre Humerusfraktur beim Kind 2014; Gercek et al. 2015) sollte eine offene Reposition nur angewendet werden bei erfolglosem geschlossenem Repositionsversuch, bei primärem Nervenschaden oder bei Durchblutungsstörungen. Nach der geschlossenen Reposition ist das gängigste Operationsverfahren das Einbringen von gekreuzten Kirschner-Drähten (gekreuzte Bohrdrahtosteosynthese mit postoperativer Ruhigstellung in Oberarmgipsschiene oder gespaltenem Oberarmgips; Ababneh et al. 1998; de las Heras et al. 2005; Schäffer et al. 2007). Bei offener Reposition muss anschließend eine Stabilisierung mittels Osteosynthese erfolgen (Schäffer et al. 2007). Dies kann ebenfalls mittels Kirschner-Drähten (K-Drähten) erfolgen, aber auch mit einem Fixateur externe, einer deszendierenden elastische-stabilen intramedullären Nagelung (ESIN) oder mit der radialen parallelen Bohrdrahtosteosynthese (Brauer et al. 2007; Kocher et al. 2007; Schäffer et al. 2007; Feng et al. 2012; Wang et al. 2012; von Laer et al. 2013; Leitlinie suprakondyläre Humerusfraktur beim Kind 2014). Während der operativen Versorgung zeigt eine Röntgenaufnahme in zwei Ebenen die korrekte Drahtlage und ob ein Torsionsfehler (Rotationssporn) nach erfolgter Reposition verblieben ist oder beseitigt wurde (von Laer et al. 2013). Die postoperative Behandlung beinhaltet regelmäßige Wundkontrollen sowie Kontrollen der Durchblutung, Motorik und Sensibilität (Leitlinie suprakondyläre Humerusfraktur beim Kind 2014). Der Oberarmstützverband sollte bis zur knöchernen Konsolidierung (drei bis vier Wochen, außer bei ESIN) angelegt bleiben. Des Weiteren wird empfohlen, eine Röntgenuntersuchung in zwei Ebenen ca. drei bis vier Wochen postoperativ durchzuführen. Eine mögliche anschließende Verordnung von Physiotherapie wird nur bei bleibender Bewegungseinschränkung gegeben sein (von Laer et al. 2013; Leitlinie suprakondyläre Humerusfraktur beim Kind 2014).

Welches Therapieverfahren gewählt wird, kommt auf die Erfahrung, auf das Können und die Meinung des Operateurs/ der Operateurin an. Von Laer et al. (2013) vertreten die Meinung, dass die Versorgung mit ESIN die aufwendigste Methode von allen darstelle (hinsichtlich Operationsdauer, Bestrahlungsdauer und zwei Anästhesien). Hingegen sind Lacher et al. (2011) und Schäffer et al. (2007) der Meinung, dass die ESIN Methode gut zur Behandlung von suprakondylären Humerusfrakturen im Kindesalter geeignet sei, da Nervenverletzungen seltener vorkämen, das Auftreten eines Cubitus varus geringer sei und 
keine Gipsschiene verwendet werden müsse (folglich freie Beweglichkeit im Ellenbogengelenk). Somit sei das Gelenk dann belastungs- bzw. übungsstabil. Allerdings weißt Schäffer et al. (2007) auf eine Besonderheit bei der ESIN Technik hin und zwar, dass die Nägel nur dann in das distale Fragment eingebracht werden können, wenn vorher exakt reponiert wurde, da die Technik nur eine Rotationsfehlstellung von max. $5-10^{\circ}$ toleriere.

\subsubsection{Komplikationen nach suprakondylären Humerusfrakturen}

Nach suprakondylären Humerusfrakturen im Kindesalter sind sechs Hauptkomplikationen bekannt (Hefti 2015):

1) Bewegungseinschränkungen: oft dauert es ca. sechs Monate, bis zum Wiedererlangen der normalen Ellenbogenbeweglichkeit (Hefti 2015). Eine Antekurvationsfehlstellung z. B. führt zur Beugeeinschränkung (Kraus et al. 2013; von Laer et al. 2013).

2) Rotationssporn: hier kommt es durch eine Verdrehung der Frakturfragmente zu einem im seitlichen Röntgenbild zumeist ventral sichtbarem Rotationssporn (von Laer et al. 2013). Abbildung 8 zeigt den Entstehungsmechanismus des Rotationsporns.
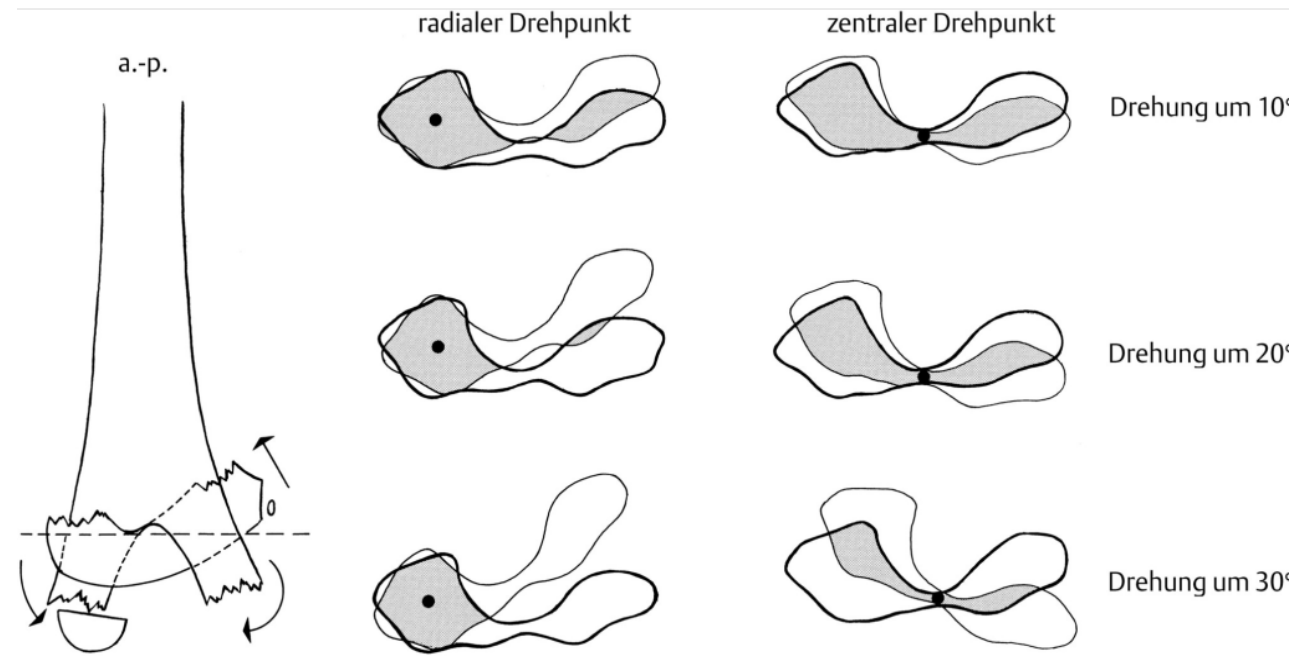

Drehung um 20
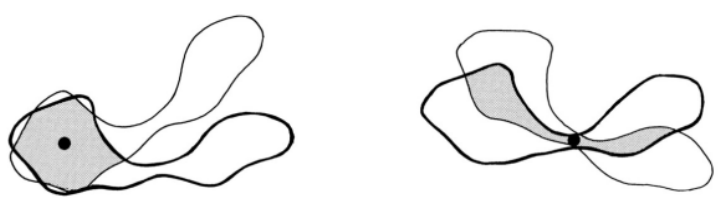

Drehung um 30

Abbildung 8: Abhängigkeit der Kontaktfläche der Fraktur von der Lage der Drehachse und dem Ausmaß einer Fehlrotation (von Laer et al. 2013). Der Abdruck erfolgt mit freundlicher Genehmigung des Thieme-Verlags

\section{3) Wachstumsstörungen}

4) Cubitus varus (Abbildung 9): der Cubitus varus ist mit 20\% Inzidenz die häufigste Komplikation (Kallio et al. 1992; von Laer 1997; Davis et al. 2000; Hefti 2015). Ursache ist der Torsionsfehler und/ oder eine ungenügende Stabilisierung der Frakturfragmente (Hefti 2015). In dem Fall liegt eine dreidimensionale Fehlstellung mit Innendrehung in der Transversalebene, Varusabkippung in der Frontalebene und Antekurvationsstellung in der Sagittalebene vor (Takeyasu et al. 2011; Kraus et al. 2013). 


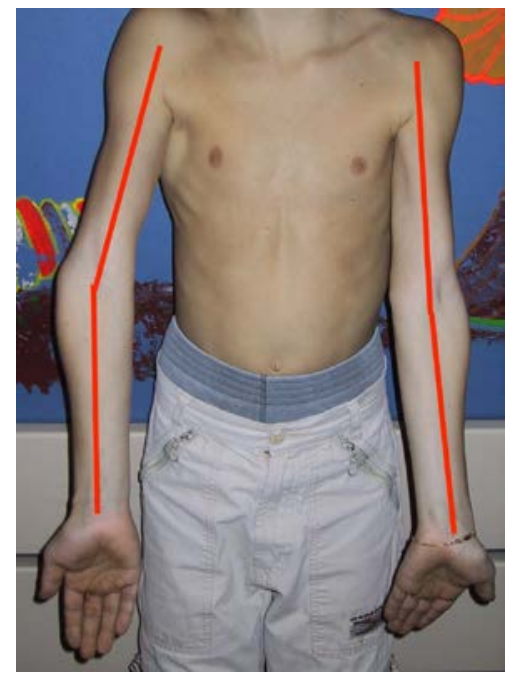

Abbildung 9: Cubitus varus auf der rechten Seite nach suprakondylärer Humerusfraktur

\section{5) Neuropathien}

6) Kompartmentsyndrom: ein Kompartmentsyndrom ist definiert als eine Druckerhöhung in einer Muskelloge, weil sich z. B. ein Hämatom entwickelt hat mit den Folgen einer Durchblutungsstörung der Muskulatur und nachfolgender Muskelnekrose und kontraktur (Niethard und Pfeil 2005). Besonders aber kann bei suprakondylären Humerusfrakturen eine Volkmann-Kontraktur auftreten. Das ist eine ischämisch bedingte Kontraktur der Armmuskulatur. Wenn die Fragmentdislokation nicht adäquat reponiert oder der Gips zu eng angelegt wurde, dann kann es zur arteriellen Ischämie und Nervenkompression im Frakturbereich mit anschließenden nekrotischen Veränderungen der Muskulatur, der Hand- und Fingerbeuger kommen (Niethard und Pfeil 2005).

\subsubsection{Der posttraumatische Rotationsfehler und seine Entwicklung am Humerus}

Um die Jahrhundertwende wurden Wachstumsstörungen für die Entstehung eines Cubitus varus verantwortlich gemacht (von Laer 1979). Heutzutage gilt der posttraumatische Torsionsfehler in der Literatur als Ursache für die Entstehung eines Cubitus varus (von Laer et al. 2013). Aber auch primäre seitliche Einstauchungen oder Abkippungen sowie radiale oder ulnare Wachstumsstörungen können eine Achsfehlstellung bewirken (von Laer 1979). Die Autoren Lubinus (1924), Aitken et al. (1943), French (1959) und Graham (1967) waren der Meinung, dass der Torsionsfehler direkt einen Einfluss auf die Ellenbogenachse habe. Hingegen dachten Flynn et al. (1974), dass der Torsionsfehler als Wegbereiter des meist ulnaren, seitlichen Abkippen des distalen Fragmentes diene. Felsenreich (1931), Arnold et al. (1977) und Mann (1963) vertraten die Ansicht, dass der Torsionsfehler keinerlei Einfluss auf den Achsenfehler habe und hielten eine seitliche Einstauchung und Abkippung verantwortlich für eine Fehlstellung im Ellenbogengelenk. 
Die klinische Bedeutung des posttraumatischen Torsionsfehlers liegt in der kosmetischen und funktionellen Einschränkung im Ellenbogengelenk (von Laer 1979). Da das Ellenbogengelenk ein Scharniergelenk ist, können Fehlstellungen in der Sagittalebene, abhängig vom Alter (Grenze ist das sechste/ siebte Lebensjahr), im weiteren Verlauf spontan korrigiert werden (Ante- und Rekurvationsfehlstellungen; von Laer 1979; von Laer et al. 2013). Eine Korrektur von Fehlstellungen in der Frontalebene, wie ein Cubitus varus z. B. wurden in der Literatur bisher nicht beschrieben (von Laer 1979; von Laer et al. 2013). Bislang sind Korrekturmöglichkeiten der Torsion nur am Femur (Morscher 1961; Oberhammer 1980; Buchholz et al. 2002) und am proximalen Drittel des Humerusschaftes in der Frontal- und Sagittalebene bekannt (von Laer et al. 2013).

Zur Untersuchung, inwiefern ein Humerustorsionsfehler im Wachstumsalter ein Korrektur- bzw. Remodelierungspotential aufweist, muss der Grad der Torsion bestimmt werden.

1979 hat von Laer an einem Untersuchungskollektiv von 203 Kindern 183 dislozierte und 20 undislozierte suprakondyläre Humerusfraktur klinisch gefunden. Bei einem radiologisch gemessenen Torsionsfehler von über $30^{\circ}$ bestand eine Abduktionshemmung im Bereich der Schulter, da das Tuberculum majus nicht nach hinten unter das Acromion gedreht werden konnte. Bei Torsionsfehlern unter $30^{\circ}$ konnte eine freie Beweglichkeit in der Schulter festgestellt werden.

\subsection{Humerustorsion}

\subsubsection{Definition der Humerustorsion/ Rotation}

In der Literatur (Hultkrantz 1904; Pieper 1982; Hefti 2015) werden die Begriffe Torsion und Rotation häufig verwechselt oder synonym gebraucht. Zur klaren Abgrenzung der Begriffe für die vorliegende Arbeit wurde daher folgende Definition herangezogen: eine Torsion ist eine Verdrehung der anatomischen Achsen der beiden Endpunkte eines Knochens in der Frontalebene gegeneinander und eine Rotation ist eine Bewegung eines Gelenkes um eine feststehende Rotationsachse (Hefti 2015). Somit wird im vorliegenden Kollektiv bei der Untersuchung der Humerusdrehung nach suprakondylären Frakturen im Wachstumsalter konsequent von einer Humerustorsion gesprochen.

\subsubsection{Bedeutung der Humerustorsion für den Oberarm}

Anatomisch betrachtet kreuzen sich eine durch das Ellenbogengelenk gelegte frontale Achse und die Projektionslinie der Oberarmkopfachse vom Tuberculum majus zur Mitte des Caput humeri in einem spitzen Winkel (14-20 ; Frick et al. 1987; Abbildung 10). 


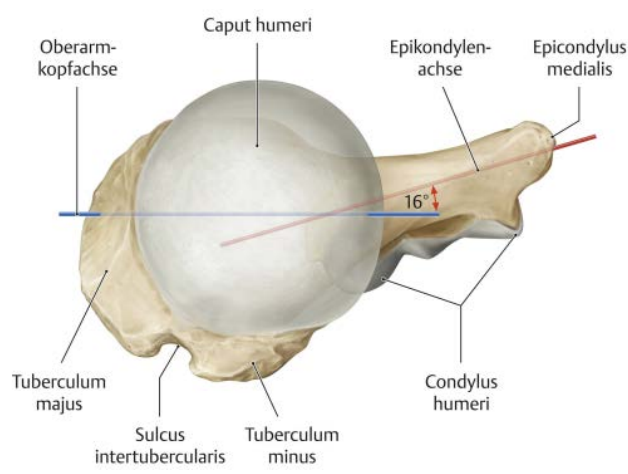

Abbildung 10: Torsion des Humerus (Schünke et al. 2011). Der Abdruck erfolgt mit freundlicher Genehmigung des Thieme-Verlags

Beim Neugeborenen beträgt die Torsion des Humerus fast $60^{\circ}$ (Frick et al. 1987). Durch die Stellungsänderung der Scapula mit Fortschreiten des Alters vermindert sich die Torsion. Grund hierfür ist die Umwandlung der Thoraxform (anfänglich fassförmig beim Neugeborenen, beim Erwachsenen queroval; Frick et al. 1987). Um die Humerustorsion zu ermitteln, gibt es unterschiedliche Messmethoden und Hilfslinienbestimmungen. Das führt jedoch zu Unterschieden der in der Literatur mitgeteilten Torsionswinkelnormwerte beim Erwachsenen (Tabelle 1):

Tabelle 1: Verschiedene Torsionswerte in der Literatur

\begin{tabular}{lcc}
\hline \multicolumn{2}{c}{ Untersucher } & $\begin{array}{c}\text { Humerustorsionswinkel } \\
\text { in Grad }\end{array}$ \\
\hline Broca & $(1881)$ & 36 \\
Gegenbaur & $(1868)$ & 37 \\
Krahl und & Evans & 37 \\
(1945) & & \\
Hultkrantz & $(1904)$ & 43 \\
Kunz et al. & $(1993)$ & 52 \\
Dähnert und & Bernd & 56 \\
(1986) & & 37 \\
Harland & $(1991)$ & 43 \\
Fuchs et al. & $(1991)$ & \\
\hline
\end{tabular}

\subsection{Torsionswinkelbestimmungen des Humerus}

In der Literatur (Gegenbaur 1868; Saha 1978; Pieper 1982; Dähnert und Bernd 1986; Harland et al. 1991) wurden unterschiedliche Messmethoden zur Torsionswinkelbe- 
stimmung des Humerus gefunden: anatomische-, röntgenologische, computertomographische- und Ultraschall Messungen.

\subsubsection{Anatomische Torsionswinkelbestimmung}

Die anatomische Torsionswinkelbestimmung wird in der Literatur kontrovers diskutiert (Gegenbaur 1868; Dähnert und Bernd 1986; Harland et al. 1991; Kunz et al. 1993). Der Humerus ist in seinem Verlauf gedreht, d. h., dass der proximale Teil des Humerus gegen den distalen nach dorsal gedreht ist (Harland et al. 1991).

Gegenbaur stellte 1868 eine anatomische Torsionswinkelbestimmung vor, indem eine proximale Achse vom Humeruskopfzentrum durch die Mitte des Tuberculum majus sowie eine distale Achse durch die Epicondylen am Humerus gelegt wurde. Beide Achsen schnitten sich in einem Punkt (Abbildung 11).

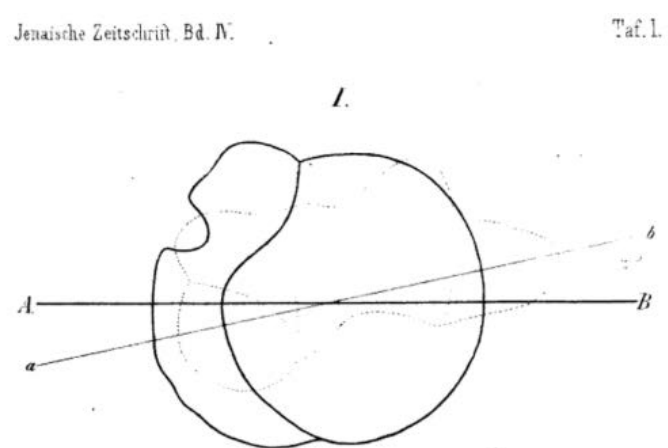

Abbildung 11: Anatomisch-anthropologischer Humerustorsionswinkel (Gegenbaur 1868) Strecke A-B: Achse durch den Gelenkkopf des Humerus. Strecke a-b: Achse durch das distale Humerusende

Bei der Messmethode nach Saha (1978) wurden insgesamt drei Achsen konstruiert. Eine Achse ging von der Mitte des proximalen Humeruskopfes durch den Punkt auf dem Tuberculum majus, der dem Sulcus intertubercularis am nächsten lag. Eine weitere Achse wurde durch den Humeruskopf gelegt und eine dritte Achse durch die distalen Epicondylen des Humerus.

\subsubsection{Radiologische Torsionswinkelbestimmung}

Nach der Methode von von Laer (von Laer 1979; von Laer et al. 2013) kann der sogenannte Rotationsfehlerquotient zur Bestimmung des Humerustorsionsfehlers herangezogen werden. Der Rotationsfehlerquotient wird bestimmt, in dem die Breite des Rotationsspornes in der seitlichen Röntgenaufnahme gemessen wird (in Millimeter) und dann durch die Gesamtbreite der Trochlea in der a.p.-Ebene geteilt wird. Bei einem 
Quotienten > 0,1 postulierten die Autoren (von Laer 1979; von Laer et al. 2013) eine Behandlung des Torsionsfehlers.

Pieper (1982) entwickelte eine spezielle Röntgentechnik, wobei der Oberarm zunächst in zwei definierten Ebenen geröntgt wurde und dann der Torsionswinkel mithilfe einer Formel berechnet wurde. Diese Formel ergab sich aus den geometrischen Beziehungen dieser beiden Ebenen zueinander.

\subsubsection{Computertomographische Torsionswinkelbestimmung}

Die Autoren Dähnert und Bernd stellten 1986 eine computertomographische Torsionswinkelbestimmung des Humerus vor. Da diese Methode jedoch Probleme bei der Oberarmlagerung und der Konstruktion der Messachsen beinhaltete, führten Fuchs et al. (1991) standardisierte Messungen durch, wobei zur Torsionswinkelbestimmung des Humerus zwei Orientierungspunkte festgelegt wurden. Die proximale Achse ging durch den tiefsten Punkt des Sulcus intertubercularis am Humeruskopf. Die beiden distalen Achsen bestanden aus zwei Tangenten an den Epicondylen: eine vordere Tangente, die an den vorderen Epicondylen und eine hintere Tangente, die an die hinteren Epicondylen gelegt wurde (Dähnert und Bernd 1986; Fuchs et al. 1991).

\subsubsection{Sonographische Bestimmung des Torsionswinkels}

Mindestens drei Autoren (Harland et al. 1991; Ito et al. 1995 und Yoshida et al. 2010) befassten sich mit der sonographischen Messung/ Erfassung des Humerustorsionswinkels. Harland et al. (1991) orientierten sich „proximal an der ventralen Kontur des Humerus mit dem Sulcus intertubercularis als markantesten Punkt" und distal an der ventralen Trochleatangente der Epicondylen. Konkret wurde zunächst der Schallkopf am Schultergelenk ventral beim liegenden Patienten aufgesetzt und der Sulcus intertubercularis waagerecht eingestellt. Auf dem Schallkopf war eine Wasserwaage montiert, welche die waagerechte Position des Schallkopfes anzeigte. Dieses Bild wurde links auf dem Monitor des Ultraschallgerätes gespeichert. Der Arm des Patienten wurde so belassen und als nächster Schritt wurde der Schallkopf kurz oberhalb des Ellenbogengelenkes aufgesetzt. Das Capitulum humeri und die Trochlea wurden hierbei sichtbar. Dieses Bild wurde auf der rechten Seite des Bildschirmes gespeichert. Anschließend wurde eine Senkrechte durch den Sulcus intertubercularis gezogen und eine Tangente an das Capitulum humeri und Trochlea gelegt. Der Schnittpunkt dieser beiden Linien wurde dann als sonographischer Retrotorsionswinkel bezeichnet (Harland et al. 1991).

Ito et al. (1995) hatten zur Messung der Humerustorsion den Patientenarm horizontal bzw. parallel an den Körper positioniert und den Ellenbogen im rechten Winkel zum Oberarm angeordnet. Die Ultraschallsonde setzte er dann über den Sulcus intertubercularis am Humerus parallel zur horizontalen Ebene auf und drehte dann die Schulter nach innen oder außen, sodass der Sulcus intertubercularis mit der Vorderseite nach oben zeigte. Hier 
war der sonographisch gemessene Torsionswinkel dann der Winkel zwischen der Längsachse des Unterarms und einer Linie senkrecht zur horizontalen Ebene.

Yoshida et al. (2010) untersuchten ebenfalls mittels Ultraschallsonde den Humerustorsionswinkel an gesunden Probanden und fanden heraus, dass die Winkel an den oberen Extremitäten zwischen dem rechten und linken Arm unterschiedlich sind. Und zwar weißt der dominante Arm eine erhöhte Humerustorsion auf, wie der nicht dominante Arm. Yoshida et al. (2010) haben im Gegensatz zu Ito et al. (1995) und Harland (1991) den Arm bei der Messung in einer Art Kasten fixiert. Das Ultraschallgerät wurde dann jeweils am Oberarm und am Ellenbogengelenk aufgesetzt und so der Torsionswinkel bestimmt.

\subsubsection{Verschiedene Messmethoden zur Bestimmung der Humerustorsion im Vergleich}

Bei den unterschiedlichen Messmethoden zur Bestimmung der Humerustorsion anatomisch, röntgenologisch, computertomographisch, sonographisch - (Gegenbaur 1868; Saha 1978; von Laer 1979; Pieper 1982; Dähnert und Bernd 1986; Fuchs et al. 1991; Harland et al. 1991; Ito et al. 1995; Yoshida et al. 2010; von Laer et al. 2013) wurden verschiedene Bezugspunkte am Humerus gewählt. Somit ergaben sich unterschiedliche Normwerte bzw. Mittelwerte des Humerustorsionswinkels. Dennoch war die Korrelation zwischen den Methoden hochsignifikant (Tabelle 2):

Tabelle 2: Vergleich der verschiedenen Humerustorsionsmessmethoden in Bezug auf deren Korrelation (modifiziert nach Harland et al. 1991)

\begin{tabular}{cc}
\hline Methode & Korrelationskoeffizient \\
\hline $\begin{array}{c}\text { röntgenologische } \\
\text { Methode nach Pieper } \\
\text { (1982) }\end{array}$ & 0,83 \\
computertomographische \\
Methode nach Dähnert \\
und Bernd (1986) \\
Sonographische Methode \\
nach Harland et al. \\
(1991)
\end{tabular}

Es traten allerdings, aufgrund der kurzen Kopf-Halsachse, am proximalen Humerus methodische Messungenauigkeiten bei der röntgenologischen und computertomographischen Bestimmung auf (Harland et al. 1991). Anzumerken ist hier, dass die Werte bei ein und demselben Patienten nicht reproduzierbar sind, da der Arm des 
Patienten in den Untersuchungen nicht fixiert wurde (außer bei der Untersuchung bei Yoshida et al. 2010) und eine Lageveränderung - auch während der Untersuchung stattfinden könnte.

Pan et al. (2015) fanden außerdem heraus, dass eine lineare Korrelation zwischen der Positionierung des Sulcus intertubercularis des Humerus und dem Humerustorsionswinkel besteht.

\subsection{Entwicklung der femoralen Antetorsion im Wachstumsalter}

Als Beispiel für eine bekannte Änderung der Torsion eines langen Röhrenknochens ist die physiologische und auch posttraumatische Korrektur im Verlauf des Wachstums am Femur bekannt (Morscher 1961; von Laer 1977; Verbeek 1979; Oberhammer 1980; Brouwer 1981; Zukunft-Huber 2011; Spyra 2011). Der Schenkelhals ist physiologisch beim Erwachsenen durchschnittlich (Ø) $15^{\circ}$ nach ventral gerichtet, was als femorale Antetorsion bezeichnet wird (Hefti 2015). Der Antetorsionswinkel wird mithilfe des Collum-Caput-DiaphysenWinkel (CCD) bestimmt. Dies ist der Winkel zwischen der Längsachse des Femurs und der Längsachse des Schenkelhalses (Zukunft-Huber 2011). Wird das Femur von kranial betrachtet und eine Linie durch die transversale Kondylenachse des distalen Femurs gezogen, bildet diese mit der Schenkelhalsachse den Antetorsionswinkel (Abbildung 12).
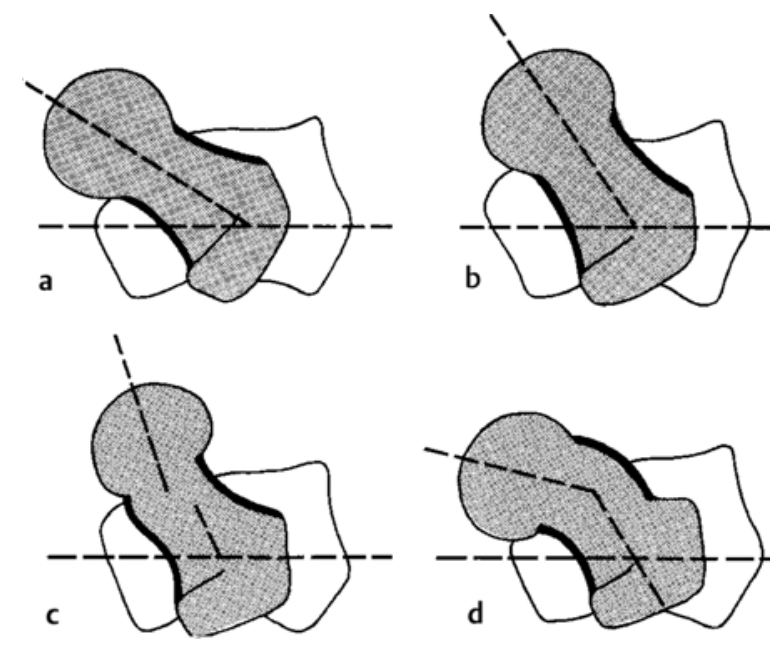

Abbildung 12: Beziehung zwischen Antetorsion des Schenkelhalses und Femurkopfkondylenquerachse: a Normale Antetorsion; b Vermehrte Antetorsion zwischen Schenkelhals und Kondylenquerachse.; c Verstärkte Antetorsion durch Antekurvation des Schenkelhalses; d Verminderte Antetorsion durch Rekurvation des Schenkelhalses (Brossmann et al. 2000). Der Abdruck erfolgt mit freundlicher Genehmigung des ThiemeVerlags

Die Antetorsion beträgt beim Neugeborenen ca. $30-35^{\circ}$, beim Erwachsenen ca. $12-15^{\circ}$ (von Lanz und Wachsmuth 1935, Hefti 2015). Diese Abnahme der Antetorsion wird durch funktionelle Aspekte und Muskelkräfte ausgelöst (Morscher 1961, Zukunft- 
Huber 2011). Durch bestimmte Krankheitsbilder (spastischen Diparese, Adduktorenspasmus, Hüftbeugekontrakturen, Lähmungen des Musculus gluteus medius) kann eine Abnahme des Antetorsionswinkel nicht erfolgen (Zukunft-Huber 2011).

Morscher (1961) ging auch bereits davon aus, dass sich die Torsionsvorgänge am Femur mechanisch erklären ließen, indem er an verschiedenen pathologisch morphologischer Zuständen am Hüftgelenk (Epiphysenlösung, Beugekontraktur, Hemiparese, Hüftgelenksluxation) forschte.

Laut den Autoren Schlickewei et al. (2006) werden verbliebene Antekurvationsstellungen im diaphysären Femurbereich $\varnothing$ um $10-15^{\circ}$ korrigiert. Bei metaphysären Frakturen des Femurs wird sogar eine Detorsion von bis zu $25^{\circ}$ gefunden (Weinberg et al. 2002). Allerdings sollten laut Schlickewei et al. (2006) Antekurvationsfehlstellungen einen Winkel von $20^{\circ}$ im Alter von fünf bis sechs Jahren nicht überschreiten.

\subsection{Fragestellung der Arbeit}

In der vorliegenden Arbeit wurden 49 Kinder nach operativ versorgter suprakondylärer Humerusfraktur in ein retrospektives $(\mathrm{N}=22)$ und ein prospektives $(\mathrm{N}=27)$ Kollektiv eingeteilt. Beide Gruppen wurden wiederholt anamnestisch, klinisch, photographisch, sonographisch und radiologisch untersucht. Hierbei wurde besonders die Entwicklung der Humerustorsion evaluiert.

Folgende Haupthypothesen wurden aufgestellt:

1. Bei Kindern mit operativ versorgter suprakondylärer Humerusfraktur kommt es oft zu einer posttraumatischen Humerustorsionsdifferenz.

2. Nach Unfall und Operation zeigt sich häufig bei Kindern mit suprakondylärer Humerusfraktur eine Änderung der posttraumtischen Humerustorsionsdifferenz.

Folgende Nebenhypothesen wurden postuliert:

1. Im Zeitraum < ein Jahr nach Unfall und Operation findet bei Kindern nach suprakondylärer Humerusfraktur mit Torsionsdifferenz eine Änderung der Humerustorsionsdifferenz statt.

2. Im Zeitraum > ein Jahr nach Unfall und Operation ist in den meisten Fällen bei Kindern nach suprakondylärer Humerusfraktur keine nennenswerte Änderung der Humerustorsionsdifferenz mehr zu erwarten. 


\section{$2 \quad$ Material und Methoden}

\subsection{Ethikkommission}

Das Studienprotokoll „Sonographische Humerustorsionsmessung bei Kindern“ eingereicht am 16.3.2006 wurde am 22.3.2006 von der Ethikkommission der Georg- August- Universität Göttingen genehmigt. Die Nummer des Ethikantrages lautet 29/2/06.

\subsection{Patientenkollektive}

Insgesamt wurden 49 Kinder mit operativ versorgten suprakondylären Humerusfrakturen im Wachstumsalter analysiert. Hierbei wurden 22 Kinder retrospektiv untersucht und 27 Kinder prospektiv evaluiert. Als Vergleichskollektiv wurden Daten aus einem Normalkollektiv ( $\mathrm{N}=99)$ ausgewertet.

Das retrospektive Kollektiv bestand aus insgesamt 22 Kindern (zwölf männliche; zehn weibliche), die in den Jahren 1996-1999 mit dislozierten suprakondylären Humerusfrakturen operativ versorgt und in den Jahren 1999, 2000 und 2001 in Jahresabständen anamnestisch, klinisch, photographisch und sonographisch nachuntersucht wurden. Die Kinder wurden in zwei Gruppen aufgeteilt. In die Gruppe retro 1 ( $\mathrm{n}=10)$ wurden diejenigen Kinder eingeschlossen, die innerhalb eines Jahres nach Fraktur ihre erste Nachuntersuchung (NU) hatten und in die Gruppe retro $2(n=12)$ die Kinder, die ihre erste NU nach einem Jahr nach Fraktur und später hatten.

In das prospektive Kollektiv wurden diejenigen Kinder $(\mathrm{N}=27 ; 16$ männliche; elf weibliche) mit vollständig dislozierter suprakondyläre Humerusfraktur eingeschlossen, die in den Jahren 2002 und 2003 operativ versorgt wurden. Anamnestische, klinische, photographische und sonographische Daten wurden sechs Wochen, vier Monate, sechs Monate und zwölf Monate nach Unfall erhoben. Neben den Röntgenaufnahmen am Unfalltag und bei der Konsolidationskontrolle (im Allgemeinen drei bis vier Wochen nach Unfall), wurde eine zusätzliche radiologische Untersuchung zur Jahreskontrolle durchgeführt.

\subsection{Normalkollektiv}

Im Jahre 2006 / 2007 wurden 50 Erwachsene und in den Jahren 1999, 2000 und 2002 insgesamt 99 Kinder nach der im Kapitel 2.5.3. sonographisch beschriebenen Methode an der oberen Extremität sonographisch untersucht. Bei 48 Kindern wurde jeweils die andere obere Extremität, die nicht von der suprakondylären Humerusfraktur betroffen war, 
untersucht. Bei 51 Kindern, die aus anderweitigen Gründen die Kinderorthopädie aufsuchten, wurden beide oberen gesunden Extremitäten gleichzeitig untersucht.

Diese Daten wurden bereits in der Publikation „Sonographische Humerustorsionsmessungen bei Kindern und Jugendlichen mit spastischer Hemiparese" von Katthagen et al. 2009 veröffentlicht und in der Dissertation Katthagen 2008 unter Verweis auf die vorliegende Arbeit publiziert.

\subsection{Inter-Observer-Messung}

Zur Validierung der sonographischen Methode wurden an insgesamt 122 (61 Probanden, davon 56 Erwachsene und fünf Kinder) oberen Extremitäten sonographische Torsionsmessungen in den Jahren 2006 und 2007 von zwei unabhängigen Untersuchern (AnnaKathrin Hell und Claudia Gadomski) durchgeführt.

\subsection{Vorgehen bei der Untersuchung}

\subsubsection{Retrospektives Kollektiv}

Retrospektiv wurden in den Jahren 1996-1999 Kinder mit dislozierten operativ versorgten suprakondylären Humerusfrakturen identifiziert $(\mathrm{N}=22)$. Aus den Patientenakten wurden folgende Daten erhoben: Unfalltag, Alter bei Fraktur, Seite der Fraktur, Typ der Fraktur, Anzahl der Tage im Krankenhaus, Anzahl der Narkosen und Komplikationen. Auch die vorhandenen Röntgenaufnahmen wurden ebenfalls mit in die Auswertung einbezogen. Auf dem seitlichen Röntgenbild wurde evaluiert, ob ein Rotationssporn vorlag und die Antwort mit ja oder nein notiert. Zusätzlich wurde bestimmt, ob eine Varus- oder Valgusfehlstellung oder ein Abkippen des Fragmentes vorlag.

Anschließend wurden die Familien telefonisch zur Teilnahme zu einer klinischen und sonographischen Nachuntersuchung eingeladen. Es wurden drei Untersuchungstermine festgelegt, die jeweils einen Abstand von einem Jahr zueinander hatten. Beim Nachuntersuchungstermin wurde das Bewegungsausmaß von Schulter und Ellenbogen nach der Neutralnullmethode erfasst und in einem Dokumentationsbogen niedergeschrieben (Abbildung 13). 


\section{Untersuchungsbogen}

Name:

Datum der Untersuchung:

Vorname:

Geburtsdatum:

\begin{tabular}{l|l|l} 
& Rechts & Links \\
\hline Schulter & & \\
Innen-/ Außenrotation & & \\
\hline Ellenbogen & & \\
Achse & & \\
Extension/ Flexion & & \\
Supination/ Pronation & & \\
\hline $\begin{array}{l}\text { Sonographie } \\
\text { Torsionswinkel im }\end{array}$ & & \\
\hline $\begin{array}{l}\text { Auffälligkeiten } \\
\text { Röntgen (falls vor- } \\
\text { handen) }\end{array}$ & & \\
\hline$\quad$
\end{tabular}

Besonderheiten:

Abbildung 13: Untersuchungsbogen für das retrospektive und prospektive Kollektiv

Werte, die über eine Neutralstellung hinausgingen (z. B. Hyperextension des Ellenbogens von $10^{\circ} ; 10-0-140$ ) wurden als negativen Wert angegeben (hier also $-10^{\circ}$ ). Bewegungsumfang und Armachsen wurden fotodokumentiert. Anschließend erfolgte die sonographische Untersuchung der Humerustorsionsmessung, welche in Kapitel 2.5.3 detailliert erläutert wird.

Im Falle einer behandlungsbedürftigen krankhaften Veränderung wurde auf Wunsch der Familie eine weiterführende Behandlung in der Klinik eingeleitet.

\subsubsection{Prospektives Kollektiv}

In den Jahren 2002 und 2003 wurden Kinder mit dislozierten operativ versorgten suprakondylären Humerusfrakturen prospektiv ( $\mathrm{N}=27)$ erfasst. Nach Aufklärung und Einwilligung der Familien wurde zusätzlich $\mathrm{zu}$ den obligatorischen klinischen und radiologischen Nachkontrollen der Fraktur bei den Untersuchungsterminen nach sechs 
Wochen, vier Monaten, sechs Monaten und zwölf Monaten sonographische Untersuchungen der Humerustorsion durchgeführt. Die allgemeinen Nachkontrollen beinhalteten die Erfassung von Problemen und Beschwerden, der Bewegungsumfänge von Schulter und Ellenbogen, eine Fotodokumentation (Abbildung 14) und eine radiologische Kontrolle nach zwölf Monaten. Auch hier wurden die Bewegungsumfänge der oberen Extremität nach der Neutralnullmethode ausgewertet (Kapitel 2.5.3, Abbildung $15 \mathrm{a}$-g). Eine Hyperextension wurde als negativer Wert angegeben.

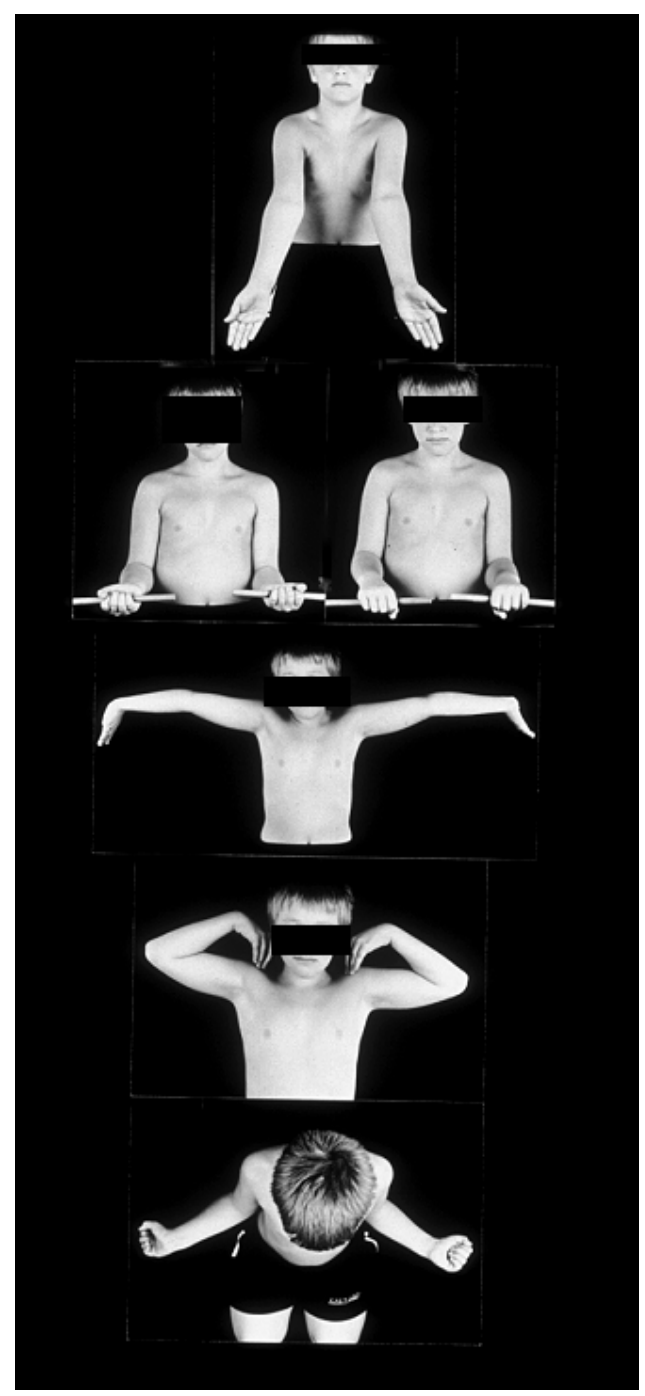

Abbildung 14: Fotodokumentation des Ellenbogenstatus: Ellenbogenachse, Supination/ Pronation, Extension, Flexion, Schulteraußenrotation (Bilder von AK Hell)

\subsubsection{Bewegungsumfänge der oberen Extremität}

Die Bewegungsumfänge der oberen Extremität für das Schulter- und Ellenbogengelenk werden mithilfe eines Winkelmessers nach der standardisierten Neutralnullmethode (von Salis-Soglio 2015) bestimmt (Abbildung 15 a-g). Mit dieser Methode kann die Beweglichkeit von Gelenken in mehreren Freiheitsgraden erfasst werden. 
Normwerte für die üblichen Bewegungsumfänge sind in mehreren Lehrbüchern angegeben (Niethard und Pfeil 2005; Niethard 2010; Imhoff und Linke 2011; Hefti 2015).

\section{Für das Schultergelenk:}

0-Stellung: Arm hängt lateral und Daumen ist nach ventral gerichtet Abduktion / Adduktion: $170^{\circ}-0-40^{\circ}$ (mit Skapulabewegung) Flexion/ Extension (Anteversion/ Retroversion): $170^{\circ}-0-40^{\circ}$

Außenrotation/ Innenrotation: bei hängendem Arm und $90^{\circ}$ Flexion im Ellenbogen: $60^{\circ}$ $0-90^{\circ}$

\section{für das Ellenbogengelenk:}

0- Stellung: Arm hängt, Daumen ist nach ventral gerichtet; Achse beträgt in Supination und voller Extension für den Mann $10^{\circ}$, für die Frau $20^{\circ}$. Kommt es zu einer Abweichung nach außen von diesen Normalwerten, wird dies als Cubitus varus und umgekehrt als Cubitus valgus bezeichnet (Abbildung 16c bzw. 16b).

Flexion/ Extension: $140^{\circ}-0-5^{\circ}$ (bei Jungen) und $140^{\circ}-0-10^{\circ}$ (bei Mädchen)

und für das Radioulnargelenk:

0-Stellung: Arm hängt, im Ellenbogen $90^{\circ}$ flektierter Arm, Unterarm zeigt horizontal nach ventral und Handgelenk in 0-Stellung

Pro- und Supination: $80^{\circ}-0-90^{\circ}$

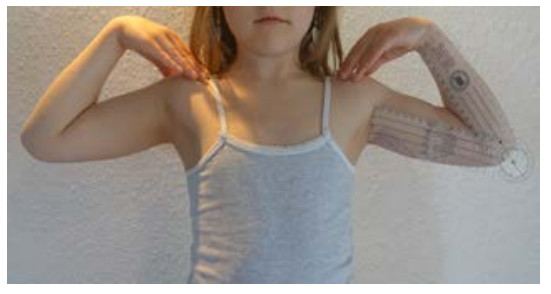

15a Flexion Ellenbogengelenk

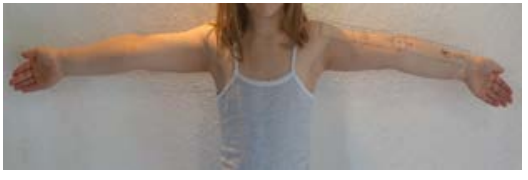

15b Extension Ellenbogengelenk

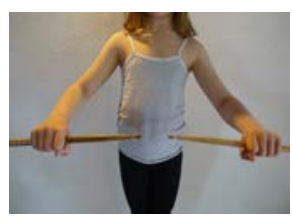

15c Pronation Ellenbogengelenk

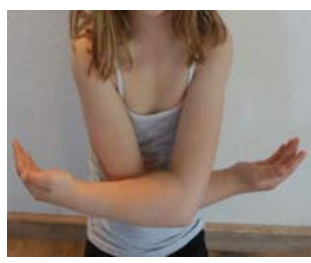

$15 f$ Innenrotation

Schultergelenk 15e Außenrotation Schultergelenk

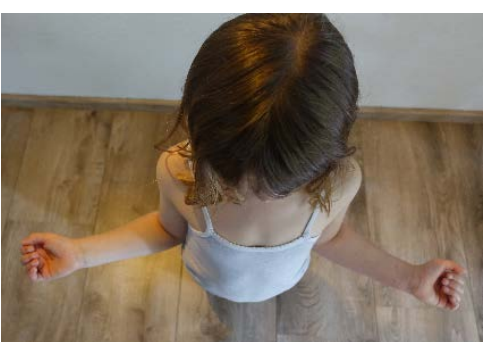

15d Supination Ellenbogengelenk

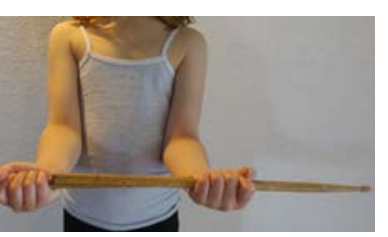




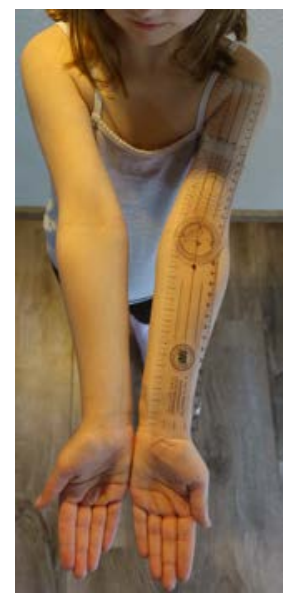

15g Ellenbogenachse

Abbildung 15a-g: Klinische Untersuchung und normaler Bewegungsumfang (Neutralnullmethode) von Schulter- und Ellenbogengelenk. In den Abbildungen 15a, 15b, 15g wurde auf den jeweils linken Arm ein Goniometer zur Darstellung der Winkelmessung gelegt

$\mathrm{Zu}$ jeden Nachuntersuchungsterminen wurden an beiden Oberarmen die Winkelgrade für die Schulterinnen- und außenrotation, Ellenbogenflexion- und extension sowie Pronation, Supination und die Ellenbogenachse bestimmt und die Differenz zwischen dem jeweils frakturierten und gesunden Arm gebildet. Bei allen Messungen wurden Unterschiede von $<10^{\circ}$ als Messfehler bewertet und nicht in die Auswertung als Abweichung eingebracht. Eine Bestimmung der Ellenbogenachse wurde nur bei kompletter Armstreckung durchgeführt.

Die Abbildung 16 zeigt einmal eine physiologische Ellenbogenachse, dann den Arm in Cubitus-valgus- und varus-Stellung.

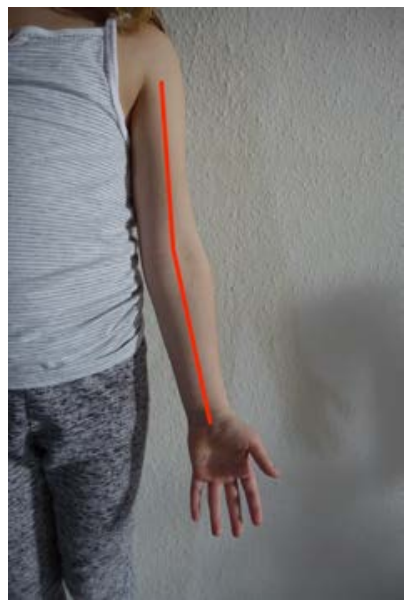

16a physiologisch

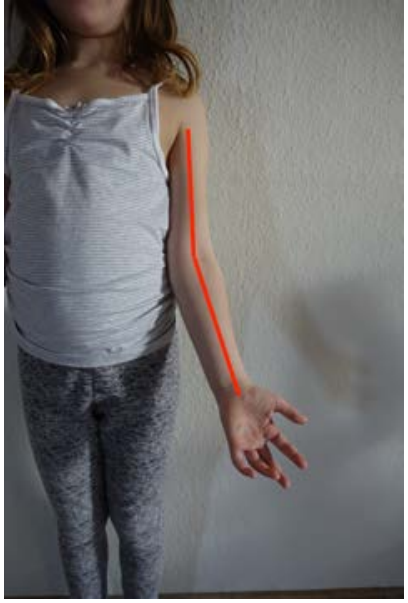

16b Cubitus valgus

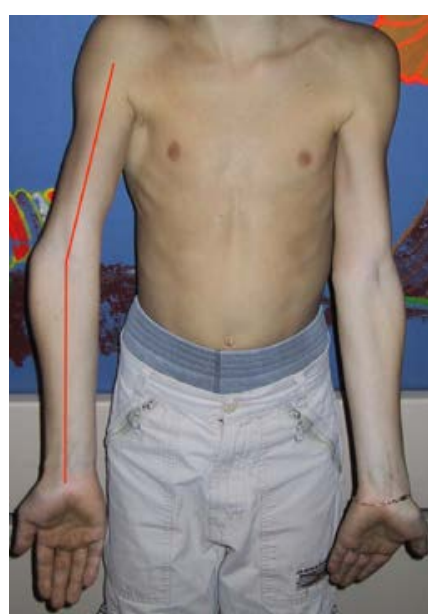

$16 c$ Cubitus varus

Abbildung 16a-c: Klinische Darstellung der physiologischen Ellenbogenachse, der Cubitus- valgus- und varus-Stellung (Bild 16c von AK Hell) 


\subsubsection{Die sonographische Untersuchung}

Die sonographischen Untersuchungen erfolgten mit einem Sonographiegerät der Marke Aloka ${ }^{\circledR}$ (SSD-1700 DynaView II; Aloka Co., Ltd. Europe Office, 2143 MZ Hoofddrop, The Netherlands) und einem 7,5-MHz-Linearschallkopf. Darauf wurde eine Wasserwaage montiert (Abbildung 17b).

Zur Untersuchung wurden die Kinder in Rückenlage auf einer Untersuchungsliege platziert. Die jeweils rechte und linke obere Extremität wurde nacheinander in eine speziell entwickelte Lagerungsschiene in $90^{\circ}$-Beugestellung fixiert (Abbildung 17a und 17c). Diese $90^{\circ}$-Fixierung schloss eine Bewegung im Ellenbogengelenk weitgehend aus. Zur Messung der Humerustorsion wurde an die Schale ein Winkelmessgerät (Plurimeter-V, Dr. Rippstein, Schweiz) montiert. Die gleiche Vorgehensweise zur Untersuchung wurde in der Dissertation von Katthagen 2008 und der Publikation Katthagen et al. 2009 beschrieben.

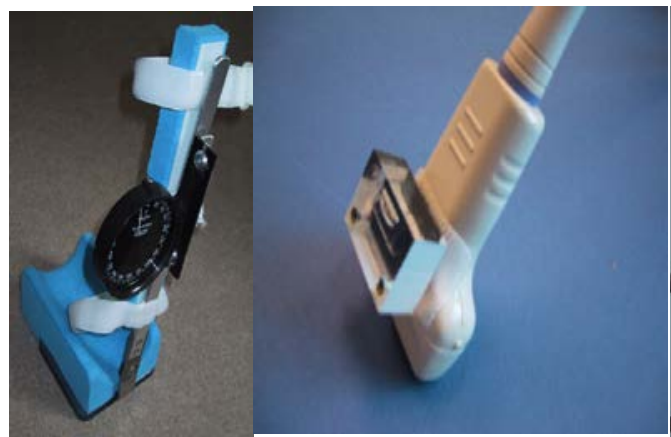

$17 \mathrm{a}$
$17 \mathrm{~b}$

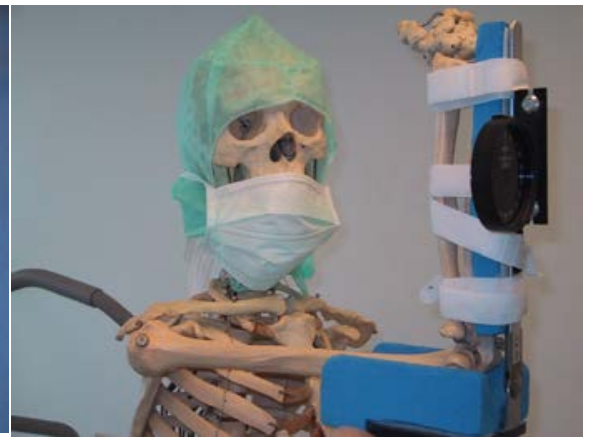

$17 \mathrm{c}$

Abbildung 17: Materialien für die Untersuchung. 17a: Lagerungsschale für die Positionierung des Armes; 17b: Linearschallkopf mit montierter Wasserwaage (Bild von AK. Hell); 17c: Lagerungsschale in Verwendung (Bild aus Katthagen et al. 2009)

Der Ellenbogen wurde in $90^{\circ}$-Beugung in die Schale gelegt und der Vorderarm in Neutralstellung fixiert, d. h. die Ulnakante lag der Schale an. Anschließend wurde mit dem Ultraschallkopf der Sulcus intertubercularis am proximalen Humeruskopf aufgesucht. Hierzu wurde der Ultraschallkopf senkrecht zur Längsachse des Humerus aufgesetzt. Die am Schallkopf befestigte Wasserwaage zeigte die waagerechte Position des Schallkopfes im Raum an. Durch Rotationsbewegungen des Armes wurden die Tubercula majus und minus so dargestellt, dass sie waagerecht in der Ultraschalldarstellung auf dem Bildschirm ausgerichtet waren. Sie bildeten mit einer Senkrechten durch den tiefsten Punkt des Sulcus intertubercularis einen $90^{\circ}$-Winkel (Abbildung 18). War der Humeruskopf nach der beschriebenen Methode eingestellt, konnte die Humerustorsion am Winkelgradmessgerät, welches an der Lagerungsschale montiert war, abgelesen werden. Hierbei diente der Vorderarm quasi als „Zeiger“ für die Ausrichtung des Kondylenmassivs, da das Ellenbogengelenk (Scharniergelenk) in der Lagerungsschale in $90^{\circ}$-Beugestellung fixiert war. 


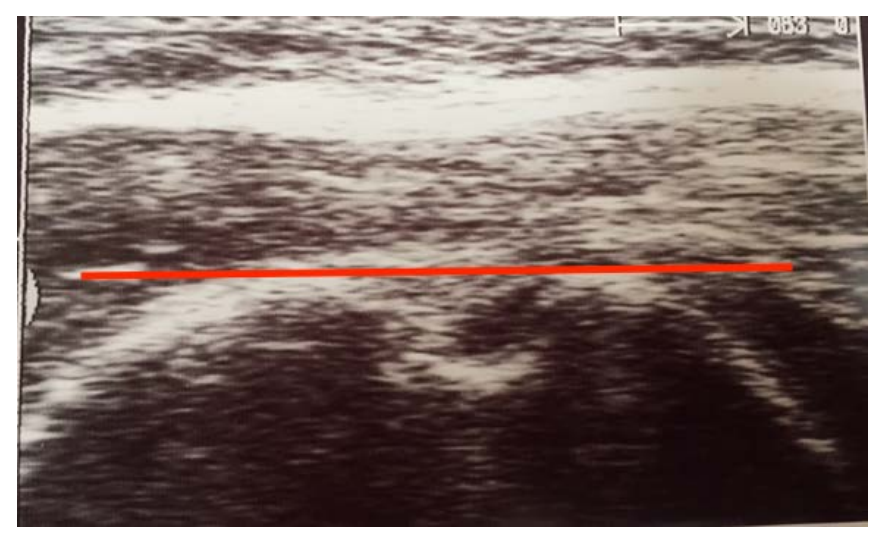

Abbildung 18: Darstellung des Sulcus intertubercularis am Humeruskopf, ventrale Schnittebene

Bei der Auswertung wurde eine klinische Innenrotation der Schulter bei waagerecht gestelltem Sulcus als positiver Humerustorsionswert notiert und umgekehrt eine klinische Außenrotation als negativer Humerustorsionswert. Um die Humerustorsionsdifferenz zu berechnen, wurde die Differenz vom frakturierten und gesunden Arm gebildet. Ein potentieller Messfehler wurde in den folgenden sonographischen Untersuchungen in den Kollektiven nicht berücksichtigt. Daten zur mittleren Abweichung unterschiedlicher Untersucher bei Durchführung der Sonographie wurden zur Validierung der Messmethode ermittelt (Kapitel 2.6).

\subsection{Validierung der Messmethode}

Zur Validierung der sonographischen Messmethode wurde an 61 Probanden (56 Erwachsene und fünf Kinder) die sonographische Torisonsmessung an beiden oberen Extremitäten (122 obere Extremitäten) in den Jahren 2006 / 2007 von zwei unterschiedlichen Untersuchern (Anna-Kathrin Hell und Claudia Gadomski) durchgeführt. Die Messungen wurden standardisiert durchgeführt: Messung der beiden oberen Extremitäten von Untersucher 1 und Notieren der Ergebnisse mit anschließendem Verlassen des Raumes. Danach selbige Durchführung durch Untersucher 2.

Diese Daten wurden ebenfalls in der Dissertation Katthagen 2008 und in der oben beschriebenen Publikation „Sonographische Humerustorsionsmessungen bei Kindern und Jugendlichen mit spastischer Hemiparese“ (Katthagen et al. 2009) beschrieben.

\subsection{Statistik und Auswertung}

Zur statistischen Auswertung der Daten wurde die Software GraphPad Prism 4.00® der Firma Graphpad Software, Inc. ${ }^{\circledR}$ (San Diego, CA, USA) verwendet. Bei einem Signifikanzniveau von $\mathrm{p}<0,05$ wurde das Ergebnis als signifikant bezeichnet. Für die Untersuchungsvariablen wurden deskriptive Statistiken berechnet, um den Mittelwert, die 
einfache Standardabweichung und die Verteilung auf Normalverteilung zu überprüfen. Dabei ist der Mittelwert das Zentrum der Verteilung des Messwerte (Field 2009). Die Standardabweichung ist der Schätzwert der mittleren Variabilität einer Variablen (Field 2009). Im Bereich \pm einer Standardabweichung um den Mittelwert sind etwa 68\% der Werte verteilt (Kubinger et al. 2011). Die angewandten Testverfahren sind in Tabelle 3 aufgelistet.

Um die absolute Übereinstimmung bei der Inter-Observer-Messung zu überprüfen, wurde die Intra-Klassen-Korrelation zwischen Untersucher 1 (Anna-Kathrin Hell) und Untersucher 2 (Claudia Gadomski) bestimmt. Dafür wurde die Software SPSS ${ }$ Statistics 21.0 für Mac der Firma IBM® (Armonk, New York, USA) verwendet.

Tabelle 3: Testbedingungen und ihre dazugehörigen Testverfahren

Bedingungen Testverfahren

Mehr als 2 abhängige Messwerte mit Normal- repeated measures ANOVA mit verteilung Bonferroni Verteilung

Mehr als 2 abhängige Messwerte ohne Friedman-Test mit Dunns Verteilung Normalverteilung

Mehr als 2 unabhängige Messwerte ohne Kruskal-Wallis-Test mit Dunns Verteilung Normalverteilung

Mehr als 2 unabhängige Messwerte mit one-way analysis of variance mit Normalverteilung Bonferroni Verteilung

2 abhängige Messwerte mit Normalverteilung

Paired t-Test

2 abhängige Messwerte ohne Normal-

Wilcoxon matched pairs Test verteilung

2 unabhängige Messwerte mit NormalUnpaired t-Test verteilung 2 unabhängige Messwerte ohne Normal- Mann-Whitney Test verteilung 


\section{Ergebnisse}

In der vorliegenden Studie wurden zur Analyse des posttraumatischen Humerustorsionsfehlers nach suprakondylären Humreusfrakturen im Kindesalter folgende Kollektive untersucht und analysiert:

- retrospektives Kollektiv mit $\mathrm{N}=22$

- prospektives Kollektiv mit $\mathrm{N}=27$

- Normalkollektiv mit N=50 Erwachsenen und N=99 Kindern (51 Kinder beide oberen Extremitäten, 48 Kinder mit der jeweilig gesunden Extremität)

- Inter-Observer-Untersuchung und Messfehlerbestimmung $\mathrm{N}=61$

\subsection{Retrospektives Kollektiv}

\subsubsection{Beschreibung des retrospektiven Kollektivs}

Die im retrospektiven Kollektiv untersuchten 22 Kinder im Alter von 1,9 bis 9,9 Jahren waren beim Unfall $\varnothing$ 6,6 \pm 2,5 Jahre alt (Abbildung 19). Zwölf Kinder waren männlich $(54,5 \%)$ und zehn Kinder weiblich (45,5\%). Bei acht der Kinder (36,4\%) war die rechte und bei $14(63,6 \%)$ die linke obere Extremität betroffen.

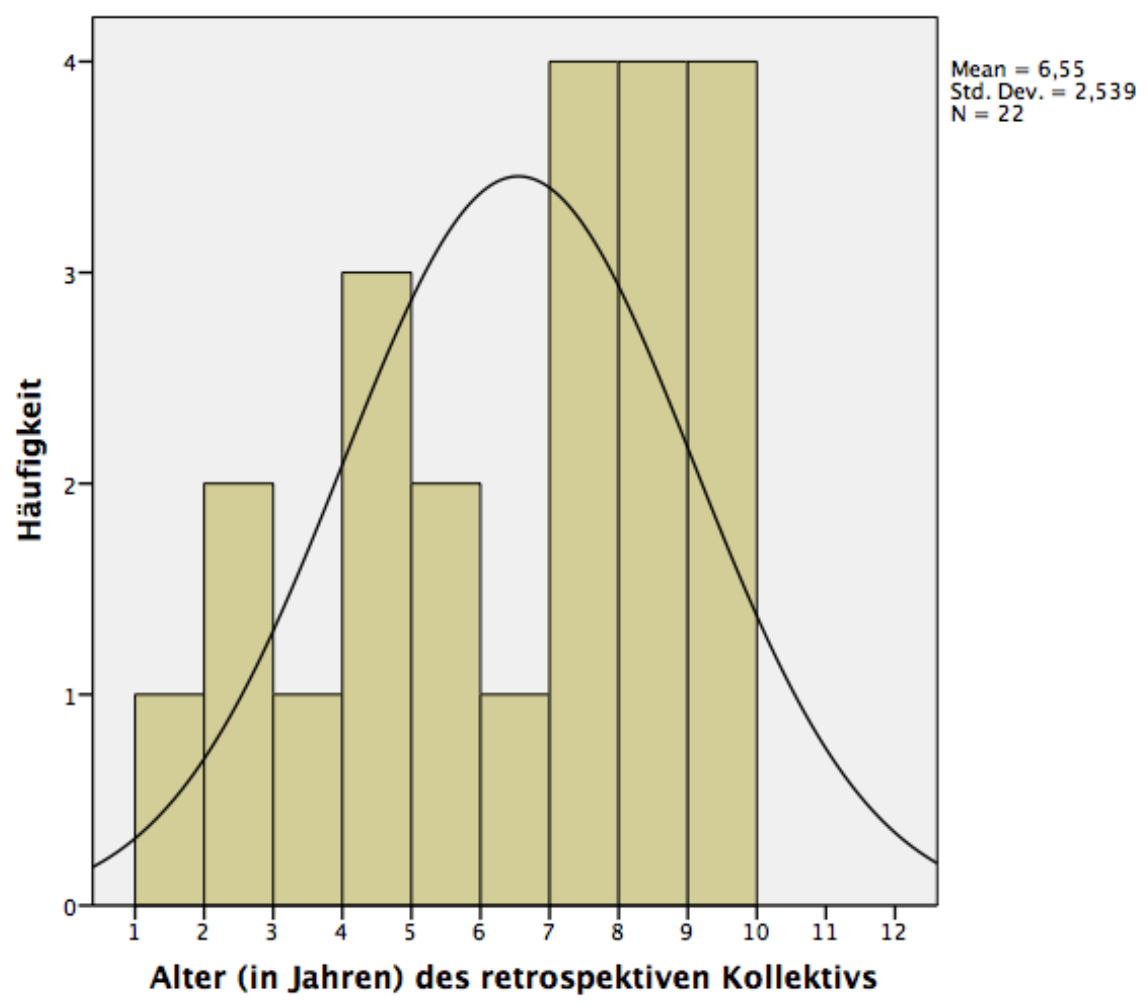

Abbildung 19: Altersverteilung des retrospektiven Kollektivs (N=22) zum Unfallzeitpunkt 
Bei einem Patienten lag eine temporäre Radialisparese der frakturierten Extremität als Begleitverletzung vor, bei einem anderen Patienten zusätzlich eine distale Radiusfraktur der gleichen Extremität. Die Mehrzahl der Kinder ( $\mathrm{n}=20$; 91\%) wurde am Unfalltag operativ versorgt, zwei Patienten wurden am fünften bzw. sechsten Tag nach dem Unfall aufgrund einer Sekundärdislokation operiert. Alle Frakturen wurden mittels geschlossener Reposition und perkutaner Fixierung mit einem radialen Fixateur externe (Howmedica Stryker ${ }^{\circledR}$, Geneva, Schweiz) behandelt (Abbildung 19). Anschließend erfolgte eine Ruhigstellung des Armes mittels einer Oberarmgipsschiene für $\varnothing 26$ Tage (17 bis 45 Tage). Nach Gipsabnahme wurde ein Konsolidationsröntgenbild angefertigt und anschließend der Fixateur externe ambulant ohne Narkose entfernt (Abbildung 20). Alle Kinder bewegten spontan die verletzte Extremität. Keiner der Patienten erhielt Krankengymnastik.
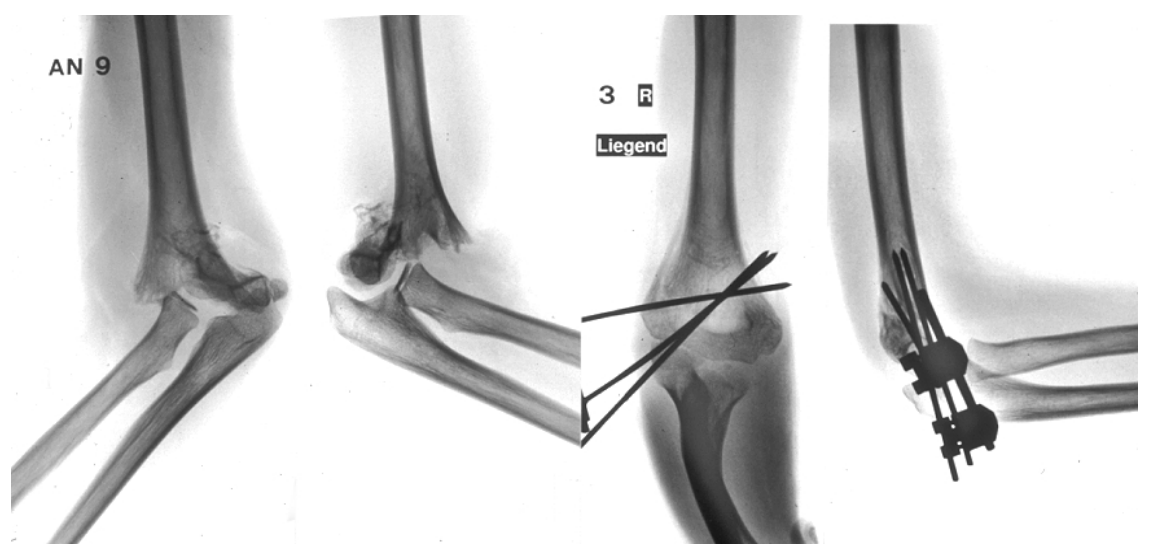

Abbildung 20: Dislozierte suprakondyläre Humerusfraktur bei einem 9-Jährigen Kind; Operative Versorgung der Fraktur mittels eines radialen Fixateur externe (Bild von AK. Hell)

Als Komplikation trat bei einem Patienten ein Pin-Infekt auf (Entzündung des umliegenden Gewebes oder des Knochens durch den Pin), der auf Gabe entsprechender Antibiotika gut ansprach. Bereits nach wenigen Tagen konnte dieser Infekt zur Abheilung gebracht werden. Bei einem anderen Patienten kam es durch einen erneuten Sturz trotz Tragens einer Oberarmgipsschiene zum Bruch eines Pins. Ein Verfahrenswechsel wurde nicht notwendig.

Der durchschnittliche Krankenhausaufenthalt lag bei 2,5 Tagen ( $\mathrm{n}=21)$, lediglich der Patient mit der Radialisparese verweilte 12 Tage im Krankenhaus. Alle Patienten erhielten nur eine Narkose, und es wurden durchschnittlich 2,1 radiologische Untersuchungen (Unfallbilder und Konsolidationsröntgen) durchgeführt.

\subsubsection{Zeitpunkte der Nachuntersuchungen}

Da einige Kinder im retrospektiven Kollektiv ihre erste NU erst nach drei Jahren hatten, wurden zwei Gruppen gebildet, um die Ergebnisse untereinander vergleichen zu können. In der Gruppe retro $1(\mathrm{n}=10)$ wurden diejenigen Kinder eingeschlossen, die innerhalb eines 
Jahres nach Unfall und Operation ihre erste NU hatten und in der Gruppe retro $2(\mathrm{n}=12)$ die Kinder, die ihre erste NU ein Jahr nach Unfall und später hatten.

Gruppe retro 1 (1.NU <1 Jahr nach Unfall): $\mathrm{n}=10$ Kinder

Gruppe retro 2 (1. NU >1 Jahr nach Unfall): n=12 Kinder

Die dann folgenden Untersuchungen (NU2 und 3) fanden im jährlichen Abstand statt. Ein Kind in der Gruppe retro 2 erschien zur dritten NU nicht.

Zum Zeitpunkt der ersten Nachuntersuchung waren die Kinder $\varnothing$ 8,0 Jahre (2,8 bis 13,1 Jahre) alt.

\subsubsection{Anamnese und subjektive Beschwerden des retrospektiven Kollektivs}

Bei den Nachuntersuchungen wurde die Unfallursache erfragt (Tabelle 4). Hierbei fanden sich Stürze aus der Höhe und Stürze bei sportlichen Aktivitäten am häufigsten.

Tabelle 4: Unfallhergang und Verteilung der Unfallmuster der Kinder des retrospektiven Kollektivs $(\mathrm{N}=22)$ bei stattgehabter suprakondylärer Humerusfraktur

\begin{tabular}{lcc}
\hline \multicolumn{1}{c}{ Ätiologie } & Gesamt & Prozent \\
\hline Sturz aus Höhe (Klettergerüst, Baum, Sprossenwand) & 6 & $27 \%$ \\
Unbeobachtete Stürze & 4 & $18 \%$ \\
Sturz von Rutschbahn & 3 & $14 \%$ \\
Sturz bei sportlichen Aktivitäten (Fahrrad fahren, Turnen, Skaten) & 6 & $27 \%$ \\
Banale Stürze (Stolpern, vom Stuhl gefallen) & 3 & $14 \%$ \\
Gesamt & 22 & $100 \%$ \\
\hline
\end{tabular}

Weiterhin wurden anamnestische Daten bei den jeweiligen Nachuntersuchungen zu Kosmetik, Schmerzen und Funktionseinschränkung erhoben (Tabelle 5). Nur ein geringer Anteil (10\%) war kosmetisch unzufrieden in der Gruppe retro 1 bei NU2. In der anderen Gruppe (retro 2) waren es $8 \%$ bei der NU2. Dies fand sich in beiden Gruppen auch bis zum Zeitpunkt der NU3. Schmerzen hatten 10\% in der Gruppe retro 1 bei NU2 und bei der Gruppe retro 2 waren es 25\% (sowohl bei NU2 als auch bei NU3). Zu Funktionseinschränkungen kam es in beiden Gruppen deutlich häufiger. 60\% der Kinder in der Gruppe retro 1 hatten zu der NU1 Einschränkungen in der Funktion, aber zur NU3 nur noch 20\%. In der Gruppe retro 2 hatten $42 \%$ bei der NU1 noch Bewegungseinschränkungen. Diese wurden zwar zur NU2 etwas geringer (17\%), stiegen aber wieder bis auf $42 \%$ bei der NU3 an. Aufgrund der geringen Fallzahl wurde auf eine genauere statistische Analyse verzichtet. 
Zusammenfassend zeigte sich, dass der kosmetische Aspekt und die Schmerzen bei fast allen Nachuntersuchungen gleich bleibend waren und die Anzahl der Kinder mit Funktionseinschränkungen im beobachteten Zeitraum abnahm (zumindest in der Gruppe retro 1).

Tabelle 5: Subjektive Beschwerden des retrospektiven Kollektivs $(\mathrm{N}=22)$ zu den jeweiligen Untersuchungsterminen; aufgeteilt nach Zeitpunkt zur NU1 im Verhältnis zum Unfall: Gruppe retro $1<1$ Jahr nach Unfall; Gruppe retro $2>1$ Jahr nach Unfall

$\begin{array}{llll}\text { Subjektive Beschwerden } & \text { NU1 } & \text { NU2 }\end{array}$

\section{Gruppe retro $1(n=10)$}

Kosmetisch störend

Schmerzen

Funktionseinschränkung

Gruppe retro $2\left(n=12^{a}\right)$

Kosmetisch störend

Schmerzen

Funktionseinschränkung
/

/

6

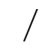

l

5
1

2
0

0

2

1

3

5

Anmerkung. $\mathrm{NU}=$ Nachuntersuchung. $/=$ keine Angabe möglich. ${ }^{2}=$ bei NU3 $\mathrm{n}=11$

\subsubsection{Klinische Untersuchung des retrospektiven Kollektivs}

Bei der klinischen Untersuchung wurden Innen- und Außenrotation der Schulter sowie Extension, Flexion, Pronation und Supination des Ellenbogens und der Achsenstatus des jeweiligen gesunden und frakturierten Armes ermittelt (Abbildung 15a-g und Kapitel 2.5).

\subsubsection{Innenrotation der Schulter im retrospektiven Kollektiv}

Die Tabelle 6 zeigt die Mittelwerte der Schulterinnenrotation jeweils auf der gesunden und frakturierten Seite sowie die Differenz der Werte der beiden Extremitäten. Es gab einen signifikanten Unterschied in der Gruppe retro 1 beim Vergleich zwischen der gesunden mit der frakturierten Seite in der NU3. In der Gruppe retro 2 gab es in den ersten beiden Nachuntersuchungen einen signifikanten Unterschied zwischen gesunder und frakturierter Seite. 
Auf der frakturierten Seite gab es zwei signifikante Unterschiede in der Gruppe retro 2 $(\mathrm{n}=11)$ zwischen der NU1 und NU3 sowie zwischen NU2 und NU3. In der Gruppe retro 1 zeigten sich keine signifikanten Unterschiede.

Für die gesunde Seite fanden sich keine signifikanten Unterschiede. Wenn die Differenz zwischen dem gesunden und frakturierten Arm gebildet wurde und dann die Mittelwerte unter den Nachuntersuchungen miteinander verglichen wurden, so zeigte sich in der Gruppe retro 1 ein signifikanter Unterschied zwischen NU1 versus NU3 und zwischen NU2 versus NU3. In der Gruppe retro 2 ergab sich kein signifikanter Unterschied zwischen den einzelnen Nachuntersuchungen. 
Tabelle 6: Ergebnisse der Untersuchung der Schulterinnenrotation in den Nachuntersuchungen im retrospektiven Kollektiv N=22 aufgeteilt nach Zeitpunkt der 1. NU im Verhältnis zum Unfall: Gruppe retro $1<1$ Jahr nach Unfall; Gruppe retro $2>1$ Jahr nach Unfall

Vergleich der gesunden mit der frakturierten Seite

Gruppe und

Untersuchungs-

zeitpunkt
Gesunde Seite Frakturierte Seite

$\mathrm{M} \pm 1 \mathrm{SD}$
Differenz frakturiertegesunde Seite

\section{Gruppe retro 1}

$(n=10)$

$\begin{array}{lllll}\text { NU1 } & 103,0 \pm 8,2^{\circ} & 105,5 \pm 12,4^{\circ} & \mathrm{p}>0,05 & 4,0 \pm 5,2^{\circ} \\ \text { NU2 } & 101,5 \pm 7,1^{\circ} & 107,5 \pm 10,1^{\circ} & \mathrm{p}>0,05 & 6,0 \pm 8,4^{\circ} \\ \text { NU3 } & 105,0 \pm 11,8^{\circ} & 99,5 \pm 10,7^{\circ} & \mathbf{p}<\mathbf{0 , 0 5} & 14,0 \pm 8,4^{\circ}\end{array}$

Vergleich $c \quad \mathrm{NU} 1=\mathrm{NU} 2=\mathrm{NU} 3 \quad \mathrm{NU} 1=\mathrm{NU} 2=\mathrm{NU} 3$

NU3 $>$ NU1 $=$ NU2

\section{Gruppe retro 2}

$(n=12)$

$\begin{array}{lllll}\text { NU1a } & 104,0 \pm 5,8^{\circ} & 111,8 \pm 5,1^{\circ} & \mathbf{p}<\mathbf{0 , 0 5} & 6,4 \pm 10,0^{\circ} \\ \text { NU2 } & 101,3 \pm 4,3^{\circ} & 109,2 \pm 7,6^{\circ} & \mathbf{p}<\mathbf{0 , 0 5} & 7,5 \pm 7,2^{\circ} \\ \text { NU3a } & 102,7 \pm 9,0^{\circ} & 95,5 \pm 10,4^{\circ} & \mathrm{p}>0,05 & 10,9 \pm 10,4^{\circ}\end{array}$

Vergleich

Anmerkung. $\mathrm{NU}=$ Nachuntersuchung. $\mathrm{M}=$ Mittelwert. $\mathrm{SD}=$ Standardabweichung. a NU1, NU3: $n=11 .{ }^{b}$ p-Wert: Paired t-Test, außer bei Gruppe retro 2 NU2. Hier Wilcoxon matched pairs Test. ${ }^{c}$ Vergleich: repeated measures ANOV A mit Bonferroni Verteilung, außer in Gruppe retro 2. Hier Friedman-Test mit Dunns Verteilung. $\mathrm{NU} 1=\mathrm{NU} 2=\mathrm{NU} 3$, wenn $\mathrm{p}>0,05$ und Zeichen $<$ bzw. $>$ bedeuten $\mathrm{p}<0,05$ 


\subsubsection{Außenrotation der Schulter im retrospektiven Kollektiv}

Auch hier wurden die jeweiligen Werte der Schulteraußenrotation auf der gesunden und frakturierten Seite bei jeder Nachuntersuchung untereinander verglichen (Tabelle 7). Hierbei zeigten sich in der Gruppe retro 1 nur bei der NU2 ein signifikanter Unterschied sowie in der Gruppe retro 2, bei NU2 und NU3 $(p<0,05)$.

Auf der frakturierten Seite in der Gruppe retro 1 gab es einen signifikanten Unterschied zwischen NU2 und NU3, jedoch keinen signifikanten Unterschied in der Gruppe retro 2. Auf der gesunden Seite fanden sich keine signifikanten Unterschiede.

Signifikante Unterschiede gab es bei den Differenzen der Werte zwischen gesunder und frakturierter Seite in der Gruppe retro 1 zwischen NU2 versus NU3 und NU1 versus NU3. Bei der Gruppe retro 2 ergab sich hierbei kein signifikanter Unterschied. 
Tabelle 7: Ergebnisse der Untersuchung der Schulteraußenrotationen in den Nachuntersuchungen im retrospektiven Kollektiv N=22 aufgeteilt nach Zeitpunkt der 1. NU im Verhältnis zum Unfall: Gruppe retro $1<1$ Jahr nach Unfall; Gruppe retro $2>1$ Jahr nach Unfall

Vergleich der gesunden mit der frakturierten Seite

Gruppe und

Untersuchungszeit-

Gesunde Seite Frakturierte Seite

Differenz frakturiertepunkt $\mathrm{M} \pm 1 \mathrm{SD}$

$\mathrm{M} \pm 1 \mathrm{SD}$

$\mathrm{p}-$ Wert $^{\mathrm{b}}$ gesunde Seite

$\mathrm{M} \pm 1 \mathrm{SD}$

\section{Gruppe retro 1}

$(n=10)$

$\begin{array}{lcccc}\text { NU1 } & 74,5 \pm 9,6^{\circ} & 69,0 \pm 15,6^{\circ} & \mathrm{p}>0,05 & 6,5 \pm 7,5^{\circ} \\ \text { NU2 } & 71,0 \pm 12,7^{\circ} & 65,5 \pm 11,7^{\circ} & \mathbf{p}<\mathbf{0 , 0 5} & 5,5 \pm 6,0^{\circ} \\ \text { NU3 } & 72,0 \pm 20,4^{\circ} & 79,5 \pm 13,8^{\circ} & \mathrm{p}>0,05 & 14,5 \pm 9,6^{\circ} \\ & & & \\ \text { Vergleich } & \mathrm{NU} 1=\mathrm{NU} 2=\mathrm{NU} 3 & \mathbf{N U} 2<\mathbf{N U} 3, & & \\ & & \mathrm{NU} 1=\mathrm{NU} 2, & & \\ & & \mathrm{NU} 1=\mathrm{NU} 1=\mathbf{N U} 2\end{array}$

\section{Gruppe retro 2}

$(n=12)$

$\begin{array}{lcccc}\text { NU1a } & 69,1 \pm 15,9^{\circ} & 59,6 \pm 20,9^{\circ} & \mathbf{p}<\mathbf{0 , 0 5} & 11,4 \pm 9,5^{\circ} \\ \text { NU2 } & 67,9 \pm 15,9^{\circ} & 59,6 \pm 17,6^{\circ} & \mathbf{p}<\mathbf{0 , 0 5} & 8,0 \pm 7,8^{\circ} \\ \text { NU3a } & 68,6 \pm 13,1^{\circ} & 70,0 \pm 18,4^{\circ} & \mathrm{p}>0,05 & 7,7 \pm 12,1^{\circ} \\ \text { Vergleichc } & \mathrm{NU} 1=\mathrm{NU} 2=\mathrm{NU} 3 & \mathrm{NU} 1=\mathrm{NU} 2=\mathrm{NU} 3 & & \mathrm{NU} 1=\mathrm{NU} 2=\mathrm{NU} 3\end{array}$

Anmerkung. $\mathrm{NU}=$ Nachuntersuchung. $\mathrm{M}=$ Mittelwert. $\mathrm{SD}=$ Standardabweichung. ${ }^{a}$ NU1, NU3: $\mathrm{n}=11 .{ }^{\mathrm{b}} \mathrm{p}$-Wert: Paired t-Test. ${ }^{\mathrm{c}}$ Vergleich: repeated measures ANOVA mit Bonferroni Verteilung. NU1 $=\mathrm{NU} 2=\mathrm{NU} 3$ wenn $\mathrm{p}>0,05$ und Zeichen $<$ bzw. $>$ bedeuten $\mathrm{p}<0,05$

Zusammenfassend ließ sich feststellen, dass das Maß der Schulterinnenrotation auf der frakturierten Seite im Laufe der Nachuntersuchungen abnahm und die Differenz zwischen der gesunden und frakturierten Seite in dieser Zeit größer wurde. Bei der 
Schulteraußenrotation nahm das $\mathrm{Maß}$ auf der frakturierten Seite im Laufe der Nachuntersuchungszeiträume zu.

Trotz vereinzelter Signifikanzen bei der Schulterinnen- und Schulteraußenrotation konnten keine konstanten Veränderungen im Nachuntersuchungszeitraum gefunden werden.

\subsubsection{Ellenbogenextension im retrospektiven Kollektiv}

Zur Bestimmung der Ellenbogenextension wurden die Werte zwischen der gesunden und frakturierten Extremität bei den jeweiligen Nachuntersuchungen untereinander verglichen. Hierbei zeigte sich, dass die Ellenbogenextension bei NU2 und NU3 in der Gruppe retro 1 an der erkrankten oberen Extremität im Gegensatz zur gesunden oberen Extremität signifikant eingeschränkt war und bei der Gruppe retro 2 bei NU1 und NU3 (Tabelle 8).

Es ergaben sich keine signifikanten Unterschiede bei der Ellenbogenextension auf der frakturierten und gesunden Seite sowie bei der Differenz in beiden Gruppen. 
Tabelle 8: Ergebnisse der Untersuchung der Ellenbogenextension bei den jeweiligen Nachuntersuchungen im retrospektiven Kollektiv N=22 aufgeteilt nach Zeitpunkt der 1. NU im Verhältnis zum Unfall: Gruppe retro $1<1$ Jahr nach Unfall; Gruppe retro $2>1$ Jahr nach Unfall

Vergleich der gesunden mit der frakturierten Seite

$\begin{array}{lcccc}\begin{array}{l}\text { Gruppe und } \\ \text { Untersuchungs- } \\ \text { zeitpunkt }\end{array} & \text { Gesunde Seite } & \text { Frakturierte Seite } & & \begin{array}{c}\text { Differenz } \\ \text { frakturierte-gesunde }\end{array} \\ & \mathrm{M} \pm 1 \mathrm{SD} & \mathrm{M} \pm 1 \mathrm{SD} & \mathrm{p}-\text { Wert }^{\mathrm{b}} & \text { Seite M } \pm 1 \mathrm{SD}\end{array}$

\section{Gruppe retro 1}

$(n=10)$

$\begin{array}{lllll}\text { NU1 } & -11,5 \pm 3,4^{\circ} & -8,5 \pm 8,2^{\circ} & \mathrm{p}>0,05 & 2,5 \pm 5,4^{\circ} \\ \mathrm{NU} 2 & -12,0 \pm 4,2^{\circ} & -15,5 \pm 5,5^{\circ} & \mathbf{p}<\mathbf{0 , 0 5} & 1,5 \pm 4,7^{\circ} \\ \mathrm{NU} 3 & -9,5 \pm 3,7^{\circ} & -12,0 \pm 4,2^{\circ} & \mathbf{p}<\mathbf{0 , 0 5} & 0 \pm 0^{\circ}\end{array}$

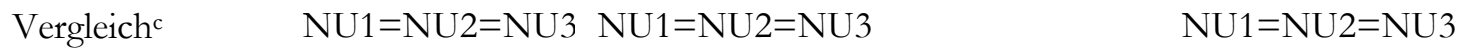

\section{Gruppe retro 2}

$(n=12)$

$\begin{array}{lcccc}\text { NU1 } & -12,5 \pm 4,5^{\circ} & -15,8 \pm 4,7^{\circ} & \mathbf{p}<\mathbf{0 , 0 5} & 1,7 \pm 3,9^{\circ} \\ \text { NU2 } & -11,2 \pm 5,0^{\circ} & -13,7 \pm 5,1^{\circ} & \mathrm{p}>0,05 & 1,7 \pm 3,9^{\circ} \\ \text { NU3a } & -8,6 \pm 4,5^{\circ} & -12,3 \pm 6,1^{\circ} & \mathbf{p}<\mathbf{0 , 0 5} & 1,8 \pm 4,0^{\circ} \\ \text { Vergleichc } & \mathrm{NU} 1=\mathrm{NU} 2=\mathrm{NU} 3 & \mathrm{NU} 1=\mathrm{NU} 2=\mathrm{NU} 3 & & \mathrm{NU} 1=\mathrm{NU} 2=\mathrm{NU} 3\end{array}$

Anmerkung. $\mathrm{NU}=$ Nachuntersuchung. $\mathrm{M}=$ Mittelwert. $\mathrm{SD}=$ Standardabweichung. - = Hyperextension.

a NU3: $\mathrm{n}=11 .{ }^{\mathrm{b}} \mathrm{p}$-Wert: Paired t-Test. ${ }^{\mathrm{c}}$ Vergleich: repeated measures ANOVA mit Bonferroni Verteilung. NU1 $=\mathrm{NU} 2=\mathrm{NU} 3$ wenn $\mathrm{p}>0,05$ und Zeichen $<$ bzw. $>$ bedeuten $\mathrm{p}<0,05$

\subsubsection{Ellenbogenflexion im retrospektiven Kollektiv}

Bei der Ellenbogenflexion zeigte sich nur in der Gruppe retro 2 bei NU1 und NU3 ein signifikanter Unterschied zwischen der gesunden und der frakturierten Seite (Tabelle 9). Die Mittelwerte der Ellenbogenflexion auf der frakturierten Seite nahmen im Verlauf 
tendenziell geringfügig zu. Die Werte der Differenz zwischen frakturierter und gesunder oberer Extremität waren eher rückläufig. Auf der gesunden und frakturierten Seite sowie bei der Differenz ergaben sich keine signifikanten Unterschiede.

Tabelle 9: Ergebnisse der Untersuchung der Ellenbogenflexion bei den jeweiligen Nachuntersuchungen im retrospektiven Kollektiv $\mathrm{N}=22$ aufgeteilt nach Zeitpunkt der 1. NU im Verhältnis zum Unfall: Gruppe retro $1<1$ Jahr nach Unfall; Gruppe retro $2>1$ Jahr nach Unfall

Vergleich der gesunden mit der frakturierten Seite

Gruppe und

Untersuchungs-

Gesunde Seite Frakturierte Seite

Differenz frakturierte-

zeitpunkt $\quad \mathrm{M} \pm 1 \mathrm{SD} \quad \mathrm{M} \pm 1 \mathrm{SD} \quad$-Wert $^{\mathrm{b}} \quad$ gesunde Seite $\mathrm{M} \pm 1 \mathrm{SD}$

\section{Gruppe retro 1}

$(\mathrm{n}=10)$

$\begin{array}{lllll}\text { NU1 } & 139,5 \pm 5,0^{\circ} & 136,5 \pm 7,1^{\circ} & \mathrm{p}>0,05 & 5,0 \pm 6,7^{\circ} \\ \mathrm{NU} 2 & 141,5 \pm 5,3^{\circ} & 139,0 \pm 10,0^{\circ} & \mathrm{p}>0,05 & 2,5 \pm 7,9^{\circ} \\ \mathrm{NU} 3 & 142,5 \pm 3,5^{\circ} & 142,0 \pm 3,5^{\circ} & \mathrm{p}>0,05 & 1,0 \pm 3,1^{\circ} \\ \text { Vergleich }^{\mathrm{c}} & \mathrm{NU} 1=\mathrm{NU} 2=\mathrm{NU} 3 & \mathrm{NU} 1=\mathrm{NU} 2=\mathrm{NU} 3 & & \mathrm{NU} 1=\mathrm{NU} 2=\mathrm{NU} 3\end{array}$

\section{Gruppe retro 2}

$(\mathrm{n}=12)$

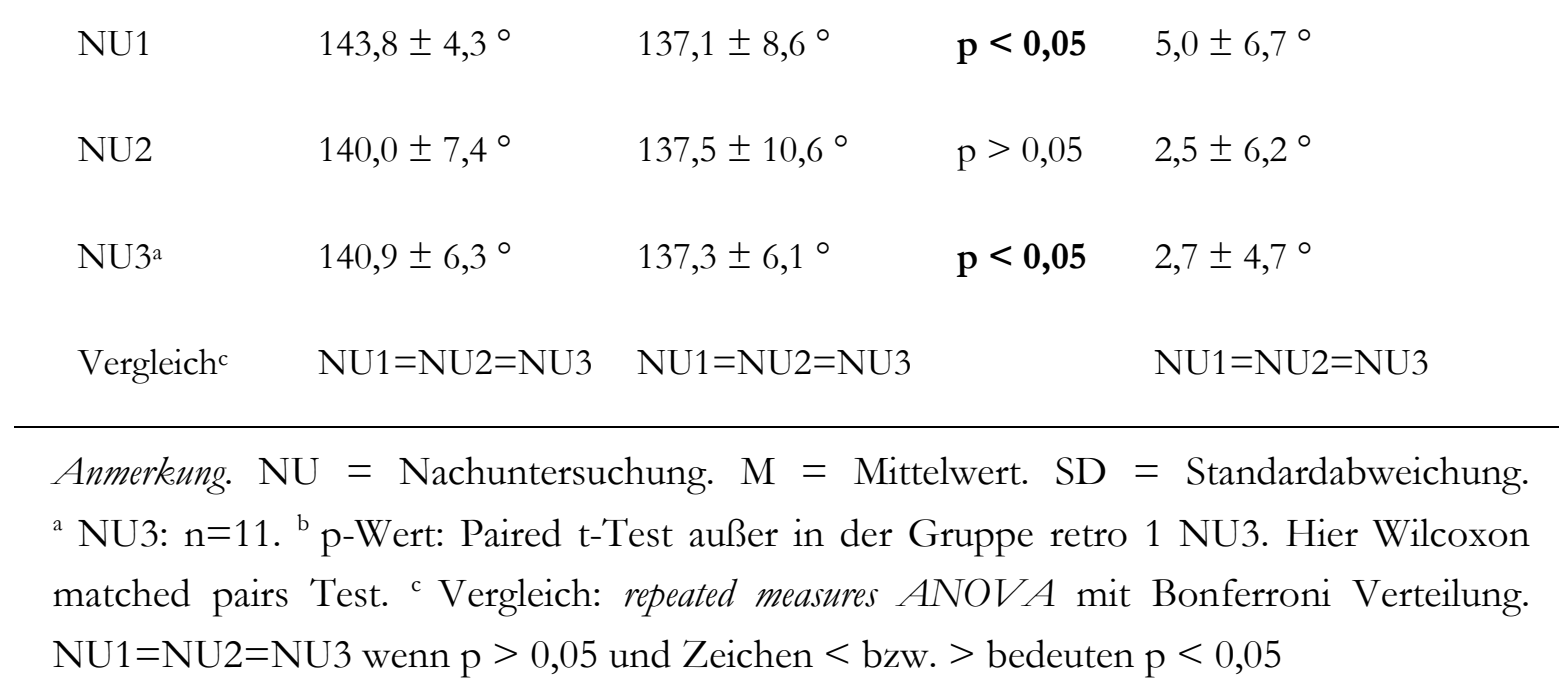




\subsubsection{Pronation des Unterarmes im retrospektiven Kollektiv}

Die Auswertung der Daten für die Pronation zeigte, dass die Kinder des retrospektiven Kollektivs hier kaum Einschränkungen aufwiesen und als Konsequenz kaum Varianzen in den Daten vorlagen. Daher konnten nur eingeschränkt die Unterschiede von gesunder und frakturierter Seite statistisch analysiert werden. Folgende Analysen waren möglich: Vergleich gesunde und frakturierte Seite in Gruppe retro 1 NU1 und Gruppe retro 2 NU3, Analyse der Veränderungen von NU1 zu NU3 auf der frakturierten Seite in Gruppe retro 1 und Gruppe retro 2 sowie die Veränderung der Differenzen zwischen gesunder und frakturierter Seite von NU1 zu NU3.

Bei der Pronation ergaben sich bei den beiden oberen Extremitäten in allen Gruppen zu keinem Zeitpunkt signifikante Unterschiede (Tabelle 10). 
Tabelle 10: Ergebnisse der Untersuchung der Pronation bei den jeweiligen Nachuntersuchungen im retrospektiven Kollektiv N=22 aufgeteilt nach Zeitpunkt der 1. NU im Verhältnis zum Unfall: Gruppe retro $1<1$ Jahr nach Unfall; Gruppe retro $2>1$ Jahr nach Unfall

Vergleich der gesunden mit der frakturierten Seite

\begin{tabular}{|c|c|c|c|c|}
\hline Gruppe und Unter- & Gesunde Seite & Frakturierte Seite & & $\begin{array}{c}\text { Differenz frakturierte- } \\
\text { gesunde Seite }\end{array}$ \\
\hline suchungszeitpunkt & $\mathrm{M} \pm 1 \mathrm{SD}$ & $\mathrm{M} \pm 1 \mathrm{SD}$ & $\mathrm{p}-\mathrm{Wert}^{\mathrm{b}}$ & $\mathrm{M} \pm 1 \mathrm{SD}$ \\
\hline
\end{tabular}

\section{Gruppe retro 1}

$(n=10)$

$\begin{array}{lcccc}\text { NU1 } & 89,0 \pm 3,2^{\circ} & 86,0 \pm 7,0^{\circ} & \mathrm{p}>0,05 & 3,0 \pm 6,7^{\circ} \\ \text { NU2 } & 90,0 \pm 0,0^{\circ} & 90,0 \pm 0,0^{\circ} & - & 0,0 \pm 0,0^{\circ} \\ \text { NU3 } & 90,0 \pm 0,0^{\circ} & 90,0 \pm 0,0^{\circ} & - & 0,0 \pm 0,0^{\circ} \\ \text { Vergleich } & \mathrm{NU} 1=\mathrm{NU} 2=\mathrm{NU} 3 & \mathrm{NU} 1=\mathrm{NU} 2=\mathrm{NU} 3 & & \mathrm{NU} 1=\mathrm{NU} 2=\mathrm{NU} 3\end{array}$

\section{Gruppe retro 2}

$$
(n=12)
$$

NU1

$90,0 \pm 0,0^{\circ}$

$87,1 \pm 10,1^{\circ}$

$2,9 \pm 10,1^{\circ}$

NU2

$89,2 \pm 2,9^{\circ}$

$89,2 \pm 2,9^{\circ}$

$0,0 \pm 0,0^{\circ}$

NU3a

$88,2 \pm 4,0^{\circ}$

$86,4 \pm 6,7^{\circ}$

$\mathrm{p}>0,05$

$3,6 \pm 5,0^{\circ}$

Vergleich $^{c}$

$\mathrm{NU} 1=\mathrm{NU} 2=\mathrm{NU} 3 \quad \mathrm{NU} 1=\mathrm{NU} 2=\mathrm{NU} 3$

$\mathrm{NU} 1=\mathrm{NU} 2=\mathrm{NU} 3$

Anmerkung. $\mathrm{NU}=$ Nachuntersuchung. $\mathrm{M}=$ Mittelwert. $\mathrm{SD}=$ Standardabweichung. - = statistische Berechnung wegen fehlender Varianz in den Daten nicht möglich.

${ }^{a}$ NU3: $n=11 .{ }^{b}$ p-Wert: Wilcoxon matched pairs Test. ${ }^{\mathrm{c}}$ Vergleich: Friedman-Test mit Dunns Verteilung. NU1 $=$ NU2 $=$ NU3 wenn $p>0,05$ 


\subsubsection{Supination des Unterarmes im retrospektiven Kollektiv}

Auch die Auswertung der Daten für die Supination zeigte, dass die Kinder des retrospektiven Kollektivs hier kaum Einschränkungen aufwiesen und als Konsequenz kaum Varianz in den Daten vorlag. Tabelle 11 gibt einen Überblick über die Ergebnisse zur Supination in den Nachuntersuchungen im retrospektiven Kollektiv. Es fanden sich in allen Berechnungen keine signifikanten Unterschiede.

Tabelle 11: Ergebnisse der Untersuchung der Supination bei den jeweiligen Nachuntersuchungen im retrospektiven Kollektiv N=22 aufgeteilt nach Zeitpunkt der 1. NU im Verhältnis zum Unfall: Gruppe retro $1<1$ Jahr nach Unfall; Gruppe retro $2>1$ Jahr nach Unfall

Vergleich der gesunden mit der frakturierten Seite

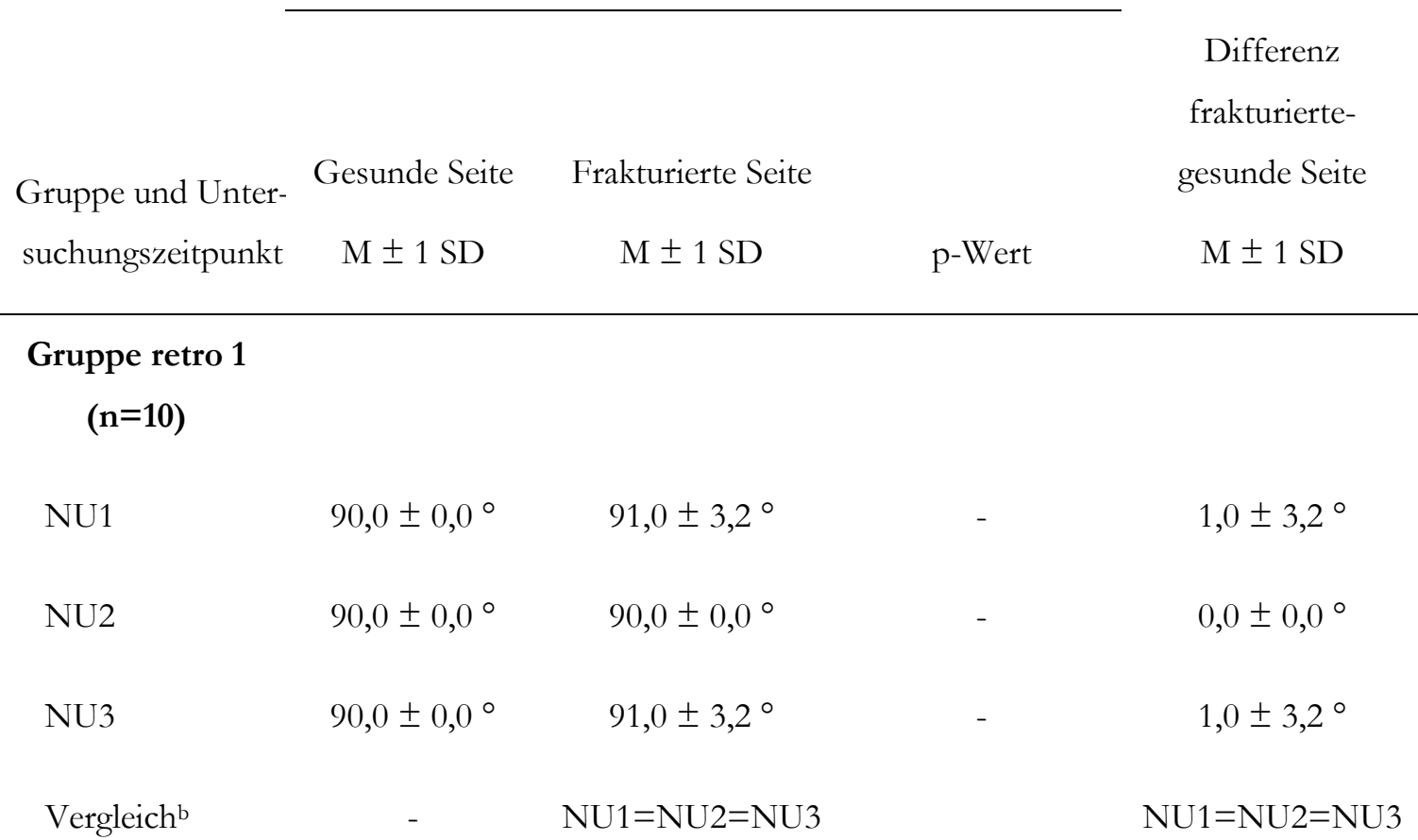

\section{Gruppe retro 2}

$(n=12)$

\begin{tabular}{|c|c|c|c|c|}
\hline NU1 & $90,0 \pm 0,0^{\circ}$ & $89,6 \pm 1,4^{\circ}$ & - & $0,0 \pm 0,0^{\circ}$ \\
\hline NU2 & $90,0 \pm 0,0^{\circ}$ & $89,6 \pm 1,4^{\circ}$ & - & $0,0 \pm 0,0^{\circ}$ \\
\hline NU3a & $90,0 \pm 0,0^{\circ}$ & $89,1 \pm 3,0^{\circ}$ & - & $0,9 \pm 3,0^{\circ}$ \\
\hline
\end{tabular}

Vergleich ${ }^{\mathrm{b}}$

$\mathrm{NU} 1=\mathrm{NU} 2=\mathrm{NU} 3$

$\mathrm{NU} 1=\mathrm{NU} 2=\mathrm{NU} 3$

Anmerkung. $\mathrm{NU}=$ Nachuntersuchung. $\mathrm{M}=$ Mittelwert. $\mathrm{SD}=$ Standardabweichung.

- = statistische Berechnung wegen fehlender Varianz in den Daten nicht möglich. 
${ }^{a}$ NU3: $n=11 .{ }^{\text {b }}$ Vergleich: Friedman-Test mit Dunns Verteilung. NU1=NU2=NU3 wenn $\mathrm{p}>0,05$

\subsubsection{Ellenbogenachse des retrospektiven Kollektivs}

Wenn bei der Ellenbogenachse eine X-Stellung (Cubitus valgus) vorlag, wurde diese als negativer Wert gerechnet und eine O-Stellung (Cubitus varus) als positiver Wert. Signifikante Unterschiede ließen sich in allen Gruppen bzw. Berechnungen nicht finden. Tabelle 12 gibt einen Überblick über die Befunde der Achsmessung im retrospektiven Kollektiv. Die Differenz nahm im Laufe der Untersuchungszeiträume tendenziell ab.

Tabelle 12: Ergebnisse der Untersuchung der Achsmessung bei den jeweiligen Nachuntersuchungen im retrospektiven Kollektiv N=22 aufgeteilt nach Zeitpunkt der 1. NU im Verhältnis zum Unfall: Gruppe retro $1<1$ Jahr nach Unfall; Gruppe retro $2>1$ Jahr nach Unfall

Vergleich der gesunden mit der frakturierten Seite

Differenz frakturierte-

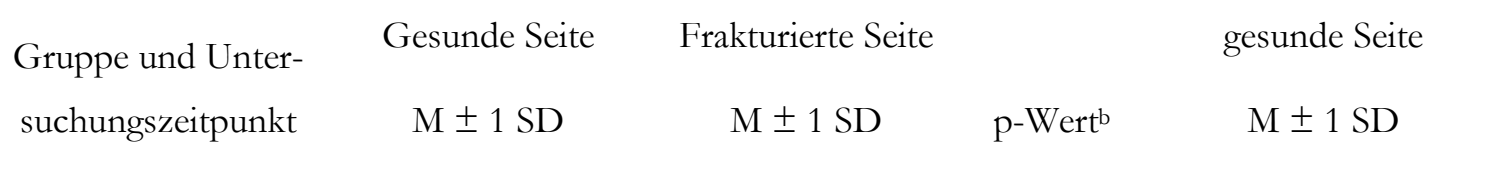

\section{Gruppe retro 1}

$\begin{array}{lllll}\text { NU1 } & -7,5 \pm 2,6^{\circ} & -2,0 \pm 12,1^{\circ} & \mathrm{p}>0,05 & 9,5 \pm 9,0^{\circ} \\ \mathrm{NU2} & -8,0 \pm 4,8^{\circ} & -1,0 \pm 13,1^{\circ} & \mathrm{p}>0,05 & 10,0 \pm 7,1^{\circ} \\ \mathrm{NU3} & -9,0 \pm 4,6^{\circ} & -6,0 \pm 9,9^{\circ} & \mathrm{p}>0,05 & 7,0 \pm 6,7^{\circ}\end{array}$

Vergleichc

$$
\mathrm{NU} 1=\mathrm{NU} 2=\mathrm{NU} 3 \quad \mathrm{NU} 1=\mathrm{NU} 2=\mathrm{NU} 3
$$

$\mathrm{NU} 1=\mathrm{NU} 2=\mathrm{NU} 3$

\section{Gruppe retro 2}

\begin{tabular}{|c|c|c|c|c|}
\hline NU1 & $-9,6 \pm 2,6^{\circ}$ & $-6,7 \pm 6,9^{\circ}$ & $\mathrm{p}>0,05$ & $4,1 \pm 6,3^{\circ}$ \\
\hline NU2 & $-9,6 \pm 3,3^{\circ}$ & $-8,3 \pm 6,9^{\circ}$ & $\mathrm{p}>0,05$ & $3,2 \pm 3,4^{\circ}$ \\
\hline $\mathrm{NU}^{\mathrm{a}}$ & $-9,1 \pm 4,4^{\circ}$ & $-9,1 \pm 4,4^{\circ}$ & $\mathrm{p}>0,05$ & $2,7 \pm 2,6^{\circ}$ \\
\hline
\end{tabular}

Vergleich $^{\mathrm{c}}$

Anmerkung. $\mathrm{NU}=$ Nachuntersuchung. $\mathrm{M}=$ Mittelwert. $\mathrm{SD}=$ Standardabweichung. ${ }^{a}$ NU3: $\mathrm{n}=11 .{ }^{\mathrm{b}} \mathrm{p}$-Wert: Paired t-Test außer in der Gruppe retro 2 NU1. Hier Wilcoxon 
matched pairs Test. ${ }^{\mathrm{c}}$ Vergleich: repeated measures ANOVA mit Bonferroni Verteilung $\mathrm{NU} 1=\mathrm{NU} 2=\mathrm{NU} 3$ wenn $\mathrm{p}>0,05$

\subsubsection{Sonographische Humerustorsionsmessung im retrospektiven Kollektiv}

Bei der sonographischen Messung der Humerustorsion (Kapitel 2.5.3) gingen nach außen gerichtete Torsionen (lateral) als negative Zahl in die Berechnung ein und nach innen (medial) gerichtete Torsionen als positive (Tabelle 13) Zahl.

Zwischen der gesunden und ehemals frakturierten Extremität gab es bei allen Nachuntersuchungen einen signifikanten Unterschied. Die Mittelwerte der frakturierten und gesunden Seite sowie die Werte bei der Differenz waren in beiden Gruppen von NU1 zu NU2 sowie von NU1 und NU3 signifikant unterschiedlich. 
Tabelle 13: Ergebnisse der Untersuchung der sonographisch gemessenen Humerustorsionswerte bei Kindern nach suprakondylärer Humerusfraktur im retrospektiven Kollektiv N=22 aufgeteilt nach Zeitpunkt der 1. NU im Verhältnis zum Unfall: Gruppe retro $1<1$ Jahr nach Unfall; Gruppe retro $2>1$ Jahr nach Unfall

Vergleich der gesunden mit der frakturierten Seite

Gruppe und

Untersuchungs-

Gesunde Seite Frakturierte Seite

Differenz frakturierte-

zeitpunkt

$\mathrm{M} \pm 1 \mathrm{SD}$

$\mathrm{M} \pm 1 \mathrm{SD}$

$\mathrm{p}-$ Wert $^{\mathrm{b}}$

gesunde Seite $\mathrm{M} \pm 1 \mathrm{SD}^{\mathrm{b}}$

\section{Gruppe retro 1}

$(n=10)$

$\begin{array}{lllll}\text { NU1 } & 13,6 \pm 11,2^{\circ} & 26,9 \pm 13,3^{\circ} & \mathbf{p}<\mathbf{0 , 0 5} & 18,5 \pm 9,0^{\circ} \\ \text { NU2 } & 22,6 \pm 8,8^{\circ} & 31,4 \pm 11,7^{\circ} & \mathbf{p}<\mathbf{0 , 0 5} & 10,4 \pm 7,6^{\circ} \\ \text { NU3 } & 22,4 \pm 9,8^{\circ} & 32,4 \pm 12,1^{\circ} & \mathbf{p}<\mathbf{0 , 0 5} & 10,2 \pm 6,3^{\circ}\end{array}$

Vergleich ${ }^{c} \quad \mathbf{N U} 1<\mathbf{N U} 2=\mathbf{N U} 3 \quad \mathbf{N U} 1<\mathbf{N U} 2=\mathbf{N U} 3$

NU1 $>$ NU2 $=$ NU3

\section{Gruppe retro 2}

$(n=12)$

$\begin{array}{lllll}\text { NU1 } & 12,0 \pm 15,9^{\circ} & 28,7 \pm 16,9^{\circ} & \mathbf{p}<\mathbf{0 , 0 5} & 17,0 \pm 10,0^{\circ} \\ \text { NU2 } & 19,1 \pm 14,4^{\circ} & 32,8 \pm 15,5^{\circ} & \mathbf{p}<\mathbf{0 , 0 5} & 13,7 \pm 11,3^{\circ} \\ \text { NU3a } & 20,9 \pm 15,3^{\circ} & 33,6 \pm 16,3^{\circ} & \mathbf{p}<\mathbf{0 , 0 5} & 13,1 \pm 13,6^{\circ}\end{array}$

Vergleich

$\mathrm{NU} 1<\mathrm{NU} 2=\mathrm{NU} 3 \quad \mathrm{NU} 1<\mathrm{NU} 2=\mathrm{NU} 3$

$\mathrm{NU} 1=\mathrm{NU} 2=\mathrm{NU} 3$

Anmerkung. $\mathrm{NU}=$ Nachuntersuchung. $\mathrm{M}=$ Mittelwert. $\mathrm{SD}=$ Standardabweichung.

a NU3: $\mathrm{n}=11 .{ }^{\mathrm{b}} \mathrm{p}$-Wert: Paired t-Test. ${ }^{\mathrm{c}}$ Vergleich: repeated measures ANOVA mit Bonferroni Verteilung. NU1 $=\mathrm{NU} 2=\mathrm{NU} 3$ wenn $\mathrm{p}>0,05$ und Zeichen $<$ bzw. $>$ bedeuten $\mathrm{p}<0,05$

Da bei den sonographischen Untersuchungen sich eine durchschnittliche Inter-ObserverVarianz von 3,6 ${ }^{\circ}$ ergab, wurde das vorliegende Kollektiv nochmals analysiert, indem eine sonographische Humerustorsionsdifferenz von $5^{\circ}$ (aufgerundeter Wert) als kein Unterschied angenommen wurde. Hier fand sich folgende Situation: 
In der Gruppe retro 1 hatten alle Kinder $(100 \%)$ bei der NU1 eine Humerustorsionsdifferenz. Zwei Jahre später (NU3) war noch eine Differenz bei $70 \% \quad(n=7)$ der Kinder vorhanden.

In der Gruppe retro 2 wiesen bei der NU1 neun Kinder (75\%) eine Humerustorsionsdifferenz auf. Bei einem Kind zeigte sich mit $6^{\circ}$ eine messfehlergrenzwertige Veränderung im Verlauf. Fünf Kinder (42\%) hatten bei der NU3 keine sonographisch vorliegende Humerustorsionsdifferenz (Tabelle 14).

Wurden zu den Nachuntersuchungszeitpunkten NU2 und NU3 die Werte von „keine Humerustorsionsdifferenz“ mit den Werten von „,vorhandener Humerustorsionsdifferenz“ in den beiden Gruppen untereinander verglichen, fanden sich signifikante Unterschiede. 
Tabelle 14: Ergebnisse der Untersuchung der sonographisch gemessenen Humerustorsionsdifferenz bei Kindern nach suprakondylärer Humerusfraktur im retrospektiven Kollektiv aufgeteilt nach vorhandener Humerustorsionsdifferenz bei Werten von $>5^{\circ}$ und keiner Humerustorsionsdifferenz, wenn Werte bei $<5^{\circ}$ lagen: Gruppe retro $1<1 \mathrm{Jahr}$ nach Unfall; Gruppe retro $2>1$ Jahr nach Unfall

\begin{tabular}{ccccc}
\hline & NU1 & NU2 & NU3 & Vergleich $^{\mathrm{b}}$ \\
Gruppenaufteilung & $\mathrm{M} \pm 1 \mathrm{SD}$ & $\mathrm{M} \pm 1 \mathrm{SD}$ & $\mathrm{M} \pm 1 \mathrm{SD}$ & \\
\hline
\end{tabular}

\section{Gruppe retro 1}

$\begin{array}{lcccc}\begin{array}{l}\text { Keine Humerustorsions- } \\ \text { differenz } \leq 5^{\circ}\end{array} & \begin{array}{c}\mathrm{n}=0 \\ 2,0 \pm 2,0^{\circ} \\ (\mathrm{n}=3)\end{array} & \begin{array}{c}3,3 \pm 1,2^{\circ} \\ (\mathrm{n}=3)\end{array} & \text { NU2=NU3 } \\ \begin{array}{l}\text { Humerustorsionsdifferenz }>5^{\circ} \\ \begin{array}{c}18,5 \pm 9,0^{\circ} \\ (\mathrm{n}=10)\end{array}\end{array} & \begin{array}{c}14,0 \pm 6,0^{\circ} \\ (\mathrm{n}=7)\end{array} & \begin{array}{c}13,1 \pm 5,0^{\circ} \\ (\mathrm{n}=7)\end{array} & \text { NU1=NU2=NU3 } \\ & - & \mathrm{p}<\mathbf{0 , 0 5} & \mathrm{p}<\mathbf{0 , 0 5}\end{array}$

\section{Gruppe retro 2}

\begin{tabular}{|c|c|c|c|c|}
\hline $\begin{array}{l}\text { Keine Humerustorsions- } \\
\text { differenz } \leq 5^{\circ}\end{array}$ & $\begin{array}{c}4,0 \pm 0,0^{\circ} \\
(n=3)\end{array}$ & $\begin{array}{c}2,6 \pm 1,7^{\circ} \\
\quad(\mathrm{n}=5)\end{array}$ & $\begin{array}{c}1,6 \pm 0,9^{\circ} \\
(n=5)\end{array}$ & $\mathrm{NU} 1=\mathrm{NU} 2=\mathrm{NU} 3$ \\
\hline Humerustorsionsdifferenz $>5^{\circ}$ & $\begin{array}{c}21,3 \pm 7,1^{\circ} \\
(n=9)\end{array}$ & $\begin{array}{c}21,6 \pm 7,6^{\circ} \\
(n=7)\end{array}$ & $\begin{array}{c}22,7 \pm 11,3^{\circ} \\
\left(\mathrm{n}=6^{\mathrm{a}}\right)\end{array}$ & $\mathrm{NU} 1=\mathrm{NU} 2=\mathrm{NU} 3$ \\
\hline $\mathrm{p}$-Wert ${ }^{\mathrm{d}}$ & - & $\mathrm{p}<0,05$ & $\mathrm{p}<0,05$ & \\
\hline
\end{tabular}

Anmerkung. $\mathrm{NU}=$ Nachuntersuchung. $\mathrm{M}=$ Mittelwert. $\mathrm{SD}=$ Standardabweichung. - = Berechnung nicht möglich. ${ }^{a} \mathrm{n}=6$ : ein Kind fehlte bei der NU3. ${ }^{\mathrm{b}}$ Vergleich: Gruppe retro 1 Keine Humerustorsionsdifferenz Mann Whitney Test, vorhandene Humerustorsionsdifferenz one-way analysis of variance mit Bonferroni Verteilung. Gruppe retro 2 Keine Humerustorsionsdifferenz Kruskal-Wallis-Test mit Dunns Verteilung; vorhandene Humerustorsionsdifferenz one way analysis of variance mit Dunns Verteilung. ${ }^{\mathrm{c}} \mathrm{p}$-Wert: MannWhitney Test. ${ }^{\mathrm{d}} \mathrm{p}$ - Wert: Unpaired $\mathrm{t}-$ Test. NU1 $=\mathrm{NU} 2=\mathrm{NU} 3$ wenn $\mathrm{p}>0,05$ und Zeichen $<$ bzw. $>$ bedeuten $\mathrm{p}<0,05$

In der Gruppe retro 1 (1. NU < 1 Jahr nach Unfall) hatten bei NU1 alle Kinder ( $\mathrm{n}=10$; $100 \%$ ) eine Humerustorsionsdifferenz von $>5^{\circ}$. In der Gruppe retro 2 (1. NU $>1 \mathrm{Jahr}$ nach Unfall) war das bei $75 \%$ der Kinder ( $\mathrm{n}=9$ bei NU1) der Fall. 


\subsubsection{Humerustorsionsänderung im retrospektiven Kollektiv}

\subsubsection{Humerustorsionsdifferenz bei der NU1 in Abhängigkeit von Ausmaß, Richtung und Alter in den Folgeuntersuchungen}

Die Tabelle 15 zeigt die Humerustorsiondifferenzänderung zwischen NU1 und NU3, also im Verlauf von zwei Jahren, in Abhängigkeit zum Alter der Kinder bei NU1. Es wurde immer die absolute Torsionsdifferenz angegeben. Die Richtung der Humerustorsionsdifferenz, also Innen- oder Außentorsion, wurde ebenfalls mit erfasst. Aufgrund der geringen Fallzahlen konnte jedoch keine konklusive statistische Auswertung erfolgen.

Bei sehr geringer Fallzahl bzw. teilweise nur einem Patienten fand tendenziell ein größeres Remodelling bei einer Humerusinnentorsionsdifferenz von $\geq 20^{\circ}$ statt. In der Gruppe retro 1, wo also Kinder ihre 1. NU innerhalb eines Jahres hatten, korrigierten jüngere Kinder (< 10 Jahre) besser, als ältere Kinder. 
Tabelle 15: Ergebnisse der Untersuchung der Humerustorsionsdifferenzänderung in Abhängigkeit vom Ausmaß der primären Torsionsdifferenz bei der NU1 und in Abhängigkeit vom Alter bei der NU1: Gruppe retro $1<1$ Jahr nach Unfall; Gruppe retro 2 $>1$ Jahr nach Unfall

\begin{tabular}{|c|c|c|c|}
\hline Torsionsdifferenz bei der & Anzahl der & $\begin{array}{l}\varnothing \text { Torsionsdifferenz- } \\
\text { Änderung zwischen }\end{array}$ & $\begin{array}{l}\varnothing \text { Torsionsdifferenz (NU1 zu } \\
\text { NU3) in Abhängigkeit vom } \\
\text { Alter bei der NU1 und vom }\end{array}$ \\
\hline NU1 & Kinder & NU1 / NU3 & Torsionsfehler \\
\hline Gruppe retro $1(n=10)$ & & & \\
\hline Außentorsion $<0^{\circ}$ & $3(30 \%)$ & $8,7^{\circ}$ & $\begin{array}{l}<5 \text { Jahre }(\mathrm{n}=1 ; 33 \%): 10^{\circ} \\
5 \text {-10 Jahre }(\mathrm{n}=2 ; 67 \%): 8^{\circ} \\
>10 \text { Jahre }(\mathrm{n}=0): /\end{array}$ \\
\hline Geringe Innentorsion $0-20^{\circ}$ & $2(20 \%)$ & $9^{\circ}$ & $\begin{array}{l}<5 \text { Jahre }(\mathrm{n}=0): / \\
5-10 \text { Jahre }(\mathrm{n}=2 ; 100 \%): 9^{\circ} \\
>10 \text { Jahre }(\mathrm{n}=0): /\end{array}$ \\
\hline Erhöhte Innentorsion $\geq 20^{\circ}$ & $5(50 \%)$ & $13,4^{\circ}$ & $\begin{array}{l}<5 \text { Jahre }(\mathrm{n}=1 ; 20 \%): 22^{\circ} \\
5 \text {-10 Jahre }(\mathrm{n}=4 ; 80 \%): 11,3^{\circ} \\
>10 \text { Jahre }(\mathrm{n}=0): /\end{array}$ \\
\hline Gruppe retro $2(n=12)$ & & & \\
\hline Außentorsion $<0^{\circ}$ & $1(8 \%)$ & $6,0^{\circ}$ & $\begin{array}{l}<5 \text { Jahre }(\mathrm{n}=0): / \\
5-10 \text { Jahre }(\mathrm{n}=0): / \\
>10 \text { Jahre }(\mathrm{n}=1 ; 100 \%): \mathbf{6}^{\circ}\end{array}$ \\
\hline Geringe Innentorsion $0-20^{\circ}$ & $6(50 \%)^{\mathrm{a}}$ & $6,0^{\circ}$ & $\begin{array}{l}<5 \text { Jahre }(\mathrm{n}=0): / \\
5-10 \text { Jahre }(\mathrm{n}=4 ; 80 \%): 5,3^{\circ} \\
>10 \text { Jahre }(\mathrm{n}=1 ; 20 \%): 8^{\circ}\end{array}$ \\
\hline Erhöhte Innentorsion $\geq 20^{\circ}$ & $5(42 \%)$ & $8,8^{\circ}$ & $\begin{array}{l}<5 \text { Jahre }(\mathrm{n}=2 ; 40 \%): 2^{\circ} \\
5 \text {-10 Jahre }(\mathrm{n}=0): / \\
>10 \text { Jahre }(\mathrm{n}=3 ; 60 \%): \mathbf{1 3 , 3 ^ { \circ }}\end{array}$ \\
\hline
\end{tabular}

Anmerkung. $\mathrm{NU}=$ Nachuntersuchung. ${ }^{\mathrm{a}} 6(50 \%)=$ Wert bei NU3 fehlt. $/=$ kein Wert vorhanden 
Tabelle 16 stellt die durchschnittliche Humerustorsionsdifferenz in den Nachuntersuchungen aufgeteilt auf die Altersgruppen dar. Auch hier zeigen sich wieder sehr geringe Fallzahlen je analysierter Einheit.

Tabelle 16: Ergebnisse der Untersuchung der Torsionsdifferenz in allen Nachuntersuchungen in Abhängigkeit vom Alter bei der 1. NU: Gruppe retro $1<1$ Jahr nach Unfall; Gruppe retro $2>1$ Jahr nach Unfall

\begin{tabular}{|c|c|c|c|c|c|}
\hline Altersgruppe & $\mathrm{n}$ & $\begin{array}{c}\text { NU1 } \\
M \pm 1 S D\end{array}$ & $\begin{array}{c}\text { NU2 } \\
M \pm 1 S D\end{array}$ & $\begin{array}{c}\text { NU3 } \\
M \pm 1 S D\end{array}$ & Vergleich ${ }^{b}$ \\
\hline \multicolumn{6}{|l|}{$\begin{array}{c}\text { Gruppe } \\
\text { retro } 1\end{array}$} \\
\hline $\begin{array}{l}\text { Kinder }<5 \\
\text { Jahre }\end{array}$ & 2 & $14,0 \pm 11,3^{\circ}$ & $11,0 \pm 7,1^{\circ}$ & $10,0 \pm 8,5^{\circ}$ & $\mathrm{NU} 1=\mathrm{NU} 2=\mathrm{NU} 3$ \\
\hline $\begin{array}{l}\text { Kinder } 5 \text { - } \\
10 \text { Jahre }\end{array}$ & 8 & $19,6 \pm 8,9^{\circ}$ & $10,3 \pm 8,2^{\circ}$ & $10,3 \pm 6,4^{\circ}$ & NU1 $>$ NU2 $=$ NU3 \\
\hline $\begin{array}{l}\text { Kinder > } \\
10 \text { Jahre }\end{array}$ & / & / & / & / & \\
\hline $\begin{array}{c}\text { Gruppe } \\
\text { retro } 2\end{array}$ & & & & & \\
\hline $\begin{array}{l}\text { Kinder }<5 \\
\text { Jahre }\end{array}$ & 2 & $26,0 \pm 0,0^{\circ}$ & $28,0 \pm 0,0^{\circ}$ & $26,0 \pm 2,8^{\circ}$ & $\mathrm{NU} 1=\mathrm{NU} 2=\mathrm{NU} 3$ \\
\hline $\begin{array}{l}\text { Kinder } 5 \text { - } \\
10 \text { Jahre }\end{array}$ & $5^{a}$ & $12,4 \pm 8,5^{\circ}$ & $10,0 \pm 6,6^{\circ}$ & $7,0 \pm 7,6^{\circ}$ & $\mathrm{NU} 1=\mathrm{NU} 2=\mathrm{NU} 3$ \\
\hline $\begin{array}{l}\text { Kinder }> \\
10 \text { Jahre }\end{array}$ & 5 & $18,8 \pm 11,7^{\circ}$ & $11,2 \pm 13,9^{\circ}$ & $12,8 \pm 17,2^{\circ}$ & $\mathrm{NU} 1=\mathrm{NU} 2=\mathrm{NU} 3$ \\
\hline Vergleich ${ }^{c}$ & & $\mathrm{NU} 1=\mathrm{NU} 2=\mathrm{NU} 3$ & $\mathrm{NU} 1=\mathrm{NU} 2=\mathrm{NU} 3$ & $\mathrm{NU} 1=\mathrm{NU} 2=\mathrm{NU} 3$ & \\
\hline
\end{tabular}

Anmerkung. $\mathrm{NU}=$ Nachuntersuchung. $\mathrm{M}=$ Mittelwert. $\mathrm{SD}=$ Standardabweichung. a 5: bei NU3 n= 4. ${ }^{\text {b }}$ Vergleich: Gruppe retro $1<5$ Jahre Friedman-Test mit Dunns Verteilung, 5 -10 Jahre: repeated measures ANOVA mit Bonferroni Verteilung. Gruppe retro 2: Friedman-Test mit Dunns Verteilung, außer bei $>10$ Jahre: repeated measures ANOVA mit Bonferroni Verteilung. ${ }^{\mathrm{c}}$ Vergleich: Gruppe retro 2: Kruskal-Wallis Test mit Dunns Verteilung. / = kein Wert vorhanden. NU1=NU2=NU3 wenn $\mathrm{p}>0,05$ und Zeichen $<$ bzw. $>$ bedeuten $\mathrm{p}<0,05$

In allen Altersgruppen kam es zu einer Detorsion (bis auf in der Gruppe retro 2 bei den $<$ 5-Jährigen) in den weiteren Nachuntersuchungen. Diese Detorsion war nur in der Gruppe retro 1 bei den 5- bis 10-Jährigen signifikant unterschiedlich. In der Gruppe retro 2 zeigte 
sich der größte Unterschied bei den > 10-Jährigen. Es ergab sich jedoch kein signifikanter Unterschied.

\subsubsection{Humerustorsionsdifferenz in Korrelation zum Cubitus varus/ valgus}

Tendenziell ging eine erhöhte Humerustorsionsdifferenz von $>5^{\circ}$ mit einer erhöhten Differenz der Ellenbogenachse einher (Tabelle 17). Signifikante Unterschiede ließen sich nicht finden.

Tabelle 17: Ergebnisse der Untersuchung der Humerustorsionsdifferenz bei der NU1 im Vergleich zur Differenz der Ellenbogenachse: Gruppe retro $1<1$ Jahr nach Unfall; Gruppe retro $2>1$ Jahr nach Unfall

\begin{tabular}{|c|c|c|c|c|c|}
\hline Humerustorsions- & & NU1 & NU2 & NU3 & Vergleich ${ }^{\mathrm{b}}$ \\
\hline differenz bei NU1 & $\mathrm{n}$ & $\mathrm{M} \pm 1 \mathrm{SD}$ & $\mathrm{M} \pm 1 \mathrm{SD}$ & $M \pm 1 S D$ & \\
\hline
\end{tabular}

\section{Gruppe retro 1}

$\begin{array}{llllll}\leq 5^{\circ} & 0 & / & / & / & / \\ >5^{\circ}\left(\varnothing 18,5^{\circ}\right) & 10 & 10,0 \pm 8,8^{\circ} & 10,0 \pm 7,1^{\circ} & 7,0 \pm 6,7^{\circ} & \mathrm{NU} 1=\mathrm{NU} 2=\mathrm{NU} 3\end{array}$

\section{Gruppe retro 2}

\begin{tabular}{|c|c|c|c|c|c|}
\hline$\leq 5^{\circ}\left(\varnothing 4^{\circ}\right)$ & 3 & $3,3 \pm 5,8^{\circ}$ & $1,7 \pm 2,9^{\circ}$ & $0,0 \pm 0,0^{\circ}$ & $\mathrm{NU} 1=\mathrm{NU} 2=\mathrm{NU} 3$ \\
\hline$>5^{\circ}\left(\varnothing 21,8^{\circ}\right)$ & 9 a & $3,9 \pm 6,5^{\circ}$ & $3,3 \pm 3,5^{\circ}$ & $3,8 \pm 2,3^{\circ}$ & $\mathrm{NU} 1=\mathrm{NU} 2=\mathrm{NU} 3$ \\
\hline Vergleich ${ }^{c}$ & & $\mathrm{NU} 1=\mathrm{NU} 2=\mathrm{NU} 3$ & $\mathrm{NU} 1=\mathrm{NU} 2=\mathrm{NU} 3$ & / & \\
\hline
\end{tabular}

Berechnung nicht möglich. $\mathrm{M}=$ Mittelwert. $\mathrm{SD}=$ Standardabweichung. a 9: bei NU3 $\mathrm{n}=8 .{ }^{\mathrm{b}}$ Vergleich: repeated measures $A N O V A$ mit Bonferroni Verteilung, außer bei Gruppe retro 2 mit einer Humerustorsionsdifferenz von $\leq 5^{\circ}$ : Friedman-Test mit Dunns Verteilung. ${ }^{c}$ Vergleich: Mann-Whitney Test. NU1 $=$ NU2 $=$ NU3 wenn $p>0,05$ und Zeichen $<$ bzw. $>$ bedeuten $\mathrm{p}<0,05$

\subsubsection{Humerustorsionsdifferenz und Funktion von Schulter/ Ellenbogen}

Einen Zusammenhang zum Ausmaß der Humerustorsionsdifferenz bei der 1. NU und zum Grad der Schulteraußenrotationsdifferenz in den folgenden Nachuntersuchungen konnte nicht ermittelt werden (Tabelle 18). In allen Fällen (bis auf Gruppe retro 1 bei Humerustorsionsdifferenz $>5^{\circ}$ ) verbesserte sich die Schulteraußenrotation bis zur 3. NU. Bei der Gruppe retro 1 mit $>5^{\circ}$ Humerustorsionsdifferenz kam es zu einem signifikanten Unterschied zwischen NU3 und NU1 sowie zwischen NU3 und NU2. 
Tabelle 18: Ergebnisse der Untersuchung der Humerustorsionsdifferenz im Verhältnis zur Differenz der Schulteraußenrotation in den NU 1-3: Gruppe retro $1<1$ Jahr nach Unfall; Gruppe retro $2>1$ Jahr nach Unfall

\begin{tabular}{lccccc}
\hline $\begin{array}{l}\text { Humerustorsionsdifferenz } \\
\text { bei NU1 }\end{array}$ & $\mathrm{n}$ & $\mathrm{NU1}$ & $\mathrm{NU} 2$ & $\mathrm{NU} 3$ & Vergleich $^{\mathrm{b}}$ \\
& & $\mathrm{M} \pm 1 \mathrm{SD}$ & $\mathrm{M} \pm 1 \mathrm{SD}$ & $\mathrm{M} \pm 1 \mathrm{SD}$ & \\
\hline
\end{tabular}

\section{Gruppe retro 1}

\begin{tabular}{|c|c|c|c|c|c|}
\hline$\leq 5^{\circ}$ & 0 & / & / & / & \\
\hline$>5^{\circ}\left(\varnothing 18,5^{\circ}\right)$ & 10 & $6,5 \pm 7,5^{\circ}$ & $5,5 \pm 6,0^{\circ}$ & $14,5 \pm 9,6^{\circ}$ & $\begin{array}{c}\text { NU3 }>\text { NU1 }= \\
\text { NU2 }\end{array}$ \\
\hline
\end{tabular}

\section{Gruppe retro 2}

$\begin{array}{lccccc}\leq 5^{\circ}\left(\varnothing 4^{\circ}\right) & 3 & 11,7 \pm 2,9^{\circ} & 3,3 \pm 5,8^{\circ} & 8,3 \pm 7,6^{\circ} & \begin{array}{c}\text { NU1=NU2= } \\ \text { NU3 }\end{array} \\ >5^{\circ}\left(\varnothing 21,8^{\circ}\right) & 9^{a} & 11,3 \pm 11,3^{\circ} & 9,4 \pm 8,1^{\circ} & 7,5 \pm 13,9^{\circ} & \begin{array}{c}\text { NU1=NU2= } \\ \text { NU3 }\end{array} \\ \text { Vergleichc } & & & & \\ & & \text { NU1 }= & \text { NU1 }= & \text { NU1 }= & \\ & \text { NU2 }= & \text { NU2 }= & \text { NU2 }= & \\ & \text { NU3 } & \text { NU3 } & \text { NU3 } & \end{array}$

Anmerkung. $\mathrm{NU}=$ Nachuntersuchung; $/=$ kein Wert vorhanden. $\mathrm{M}=$ Mittelwert. $\mathrm{SD}=$ Standardabweichung.

${ }^{\mathrm{a}} 9=8$ Kinder bei NU1 und NU3. ${ }^{\mathrm{b}}$ Vergleich: repeated measures ANOV A mit Bonferroni Verteilung, außer bei Gruppe retro 2 mit einer Humerustorsionsdifferenz von $\leq 5^{\circ}$ : Friedman-Test mit Dunns Verteilung. ${ }^{\mathrm{c}}$ Vergleich: Mann-Whitney Test. NU1=NU2=NU3 wenn $\mathrm{p}>0,05$ und Zeichen $<$ bzw. $>$ bedeuten $\mathrm{p}<0,05$

Bei der Humerustorsionsdifferenz bei der NU1 in Korrelation zu den Schulterinnenrotationen in den nachfolgenden Nachuntersuchungen ergab sich kein Zusammenhang (Tabelle 19). Nur in der Gruppe retro 1 mit einer Torsionsdifferenz von $>5^{\circ} \mathrm{kam}$ es zu einem signifikanten Unterschied zwischen NU1 und NU3. 
Tabelle 19: Ergebnisse der Untersuchung der Humerustorsionsdifferenz bei der NU1 im Verhältnis zur Differenz der Schulterinnenrotation in den Nachuntersuchungen 1-3: Gruppe retro $1<1$ Jahr nach Unfall; Gruppe retro $2>1$ Jahr nach Unfall

\begin{tabular}{|c|c|c|c|c|}
\hline \multirow{2}{*}{$\begin{array}{l}\text { Humerustorsionsdifferenz } n \\
\text { bei NU1 }\end{array}$} & NU1 & NU2 & NU3 & \multirow[t]{2}{*}{ Vergleich ${ }^{\mathrm{b}}$} \\
\hline & $\mathrm{M} \pm 1 \mathrm{SD}$ & $\mathrm{M} \pm 1 \mathrm{SD}$ & $\mathrm{M} \pm 1 \mathrm{SD}$ & \\
\hline
\end{tabular}

\section{Gruppe retro 1}

$\begin{array}{lllll}\leq 5^{\circ} & 0 & / & / & / \\ >5^{\circ}\left(\varnothing 18,5^{\circ}\right) & 10 & 4,0 \pm 5,2^{\circ} & 6,0 \pm 8,4^{\circ} & 14,0 \pm 8,4^{\circ}\end{array}$

NU1<NU3

$\mathrm{NU} 1=\mathrm{NU} 2$

$\mathrm{NU} 2=\mathrm{NU} 3$

\section{Gruppe retro 2}

\begin{tabular}{|c|c|c|c|c|c|}
\hline$\leq 5^{\circ}\left(\varnothing 4^{\circ}\right)$ & 3 & $5,0 \pm 8,7^{\circ}$ & $3,3 \pm 5,8^{\circ}$ & $6,7 \pm 5,8^{\circ}$ & $\begin{array}{l}\text { NU1=NU2= } \\
\text { NU3 }\end{array}$ \\
\hline$>5^{\circ}\left(\varnothing 21,8^{\circ}\right)$ & $9^{a}$ & $6,9 \pm 11,0^{\circ}$ & $8,9 \pm 7,4^{\circ}$ & $12,5 \pm 11,7^{\circ}$ & $\begin{array}{l}\text { NU1=NU2= } \\
\text { NU3 }\end{array}$ \\
\hline Vergleich ${ }^{c}$ & & $\begin{array}{l}\mathrm{NU} 1= \\
\mathrm{NU} 2=\mathrm{NU} 3\end{array}$ & $\begin{array}{l}\mathrm{NU} 1= \\
\mathrm{NU} 2=\mathrm{NU} 3\end{array}$ & $\begin{array}{l}\mathrm{NU} 1= \\
\mathrm{NU} 2=\mathrm{NU} 3\end{array}$ & \\
\hline
\end{tabular}

Anmerkung. $\mathrm{NU}=$ Nachuntersuchung. $/=$ kein Wert vorhanden. $\mathrm{M}=$ Mittelwert. $\mathrm{SD}=$ Standardabweichung.

a 9: 8 Kinder bei NU1 und NU3. ${ }^{\mathrm{b}}$ Vergleich: repeated measures ANOVA mit Bonferroni Verteilung, außer bei Gruppe retro 2 mit einer Humerustorsionsdifferenz von $\leq 5^{\circ}$ : Friedman-Test mit Dunns Verteilung. ${ }^{\mathrm{c}}$ Vergleich: Mann-Whitney Test. NU1=NU2=NU3 wenn $\mathrm{p}>0,05$ und Zeichen $<$ bzw. $>$ bedeuten $\mathrm{p}<0,05$

Bei der Ellenbogenextension bestand ebenfalls kein Zusammenhang zur Humerustorsionsdifferenz bei der 1. NU (Tabelle 20). Es gab in allen Gruppen keine signifikanten Unterschiede. Weitere statistische Berechnungen bzw. Vergleiche waren wegen der nicht vorhandenen Differenz in der Gruppe retro 2 mit einer Humerustorsionsdifferenz von $\leq 5^{\circ}$ nicht möglich. 
Tabelle 20: Ergebnisse der Untersuchung der Humerustorsionsdifferenz bei der NU1 im Vergleich zur Differenz der Ellenbogenextension in den Nachuntersuchungen: Gruppe retro $1<1$ Jahr nach Unfall; Gruppe retro $2>1$ Jahr nach Unfall

\begin{tabular}{cllll}
\hline $\begin{array}{c}\text { Humerustorsionsdifferenz } \\
\text { bei NU1 }\end{array}$ & NU1 & NU2 & NU3 & Vergleich $^{\mathrm{b}}$ \\
\hline
\end{tabular}

Gruppe retro 1

\begin{tabular}{|c|c|c|c|c|c|}
\hline$\leq 5^{\circ}$ & 0 & / & / & / & \\
\hline$>5^{\circ}\left(\varnothing 18,5^{\circ}\right)$ & 10 & $2,5 \pm 5,4^{\circ}$ & $1,5 \pm 4,7^{\circ}$ & $0,0 \pm 0,0^{\circ}$ & $\begin{array}{l}\mathrm{NU} 1= \\
\mathrm{NU} 2=\mathrm{NU} 3\end{array}$ \\
\hline
\end{tabular}

\section{Gruppe retro 2}

\begin{tabular}{|c|c|c|c|c|}
\hline$\leq 5^{\circ}\left(\varnothing 4^{\circ}\right)$ & $30,0 \pm 0,0^{\circ}$ & $0,0 \pm 0,0^{\circ}$ & $0,0 \pm 0,0^{\circ}$ & / \\
\hline$>5^{\circ}\left(\varnothing 21,8^{\circ}\right)$ & $9^{a} 2,2 \pm 4,4^{\circ}$ & $2,2 \pm 4,4^{\circ}$ & $2,5 \pm 4,6^{\circ}$ & $\begin{array}{l}\text { NU1=NU2 } \\
=\mathrm{NU} 3\end{array}$ \\
\hline
\end{tabular}

Anmerkung. NU = Nachuntersuchung. $/=$ kein Wert vorhanden bzw. statistische Berechnung nicht möglich. $\mathrm{M}=$ Mittelwert. $\mathrm{SD}=$ Standardabweichung. a 9: 8 Kinder bei NU3. ${ }^{\text {b }}$ Vergleich: statistische Berechnung mittels Friedman-Test mit Dunns Verteilung. NU1 $=$ NU2 $=$ NU3 wenn $\mathrm{p}>0,05$ und Zeichen $<$ bzw. $>$ bedeuten $\mathrm{p}<0,05$

Ein Zusammenhang zwischen der Humerustorsionsdifferenz bei der NU1 zur Ellenbogenflexion in den Nachuntersuchungen konnte in beiden Gruppen nicht gefunden werden (Tabelle 21). 
Tabelle 21: Ergebnisse der Untersuchung der Humerustorsionsdifferenz bei der NU1 im Vergleich zur Differenz der Ellenbogenflexion in den Nachuntersuchungen: Gruppe retro $1<1$ Jahr nach Unfall; Gruppe retro $2>1$ Jahr nach Unfall

\begin{tabular}{cclll}
\hline $\begin{array}{c}\text { Humerustorsionsdifferenz } \\
\text { bei NU1 }\end{array}$ & $\mathrm{NU}$ & $\mathrm{NU}$ & $\mathrm{NU}$ & Vergleich $^{\mathrm{b}}$ \\
\hline
\end{tabular}

\section{Gruppe retro 1}

$\begin{array}{llllll}\leq 5^{\circ} & 0 & / & / & / & \\ >5^{\circ}\left(\varnothing 18,5^{\circ}\right) & 10 & 5,0 \pm 6,7^{\circ} & 2,5 \pm 7,9^{\circ} & 1,0 \pm 3,2^{\circ} & \text { NU1=NU2 }\end{array}$

\section{Gruppe retro 2}

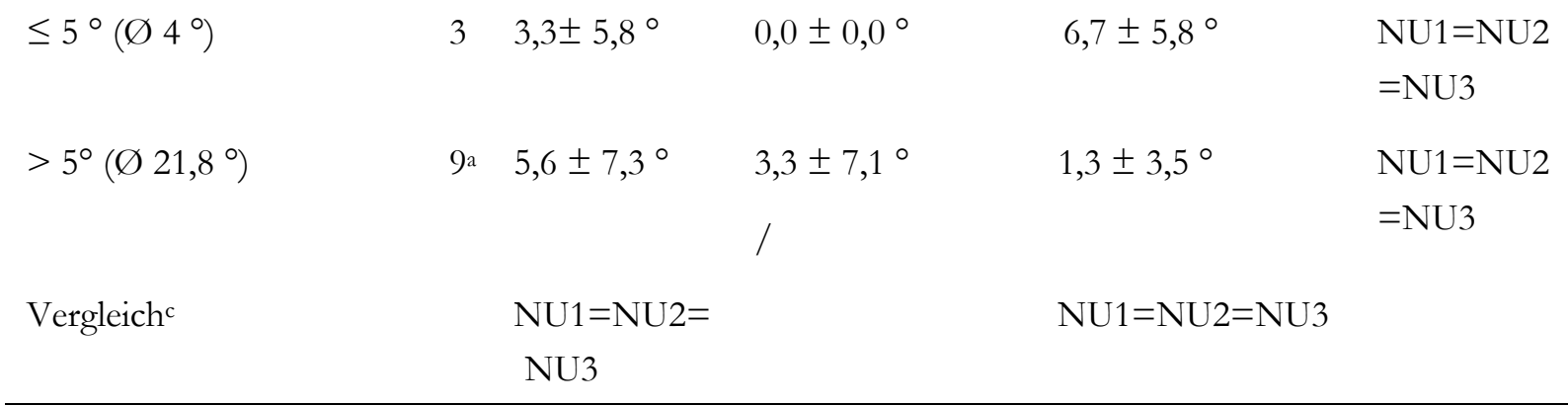

Anmerkung. $\mathrm{NU}=$ Nachuntersuchung. $/=$ kein Wert vorhanden bzw. statistische Berechnung nicht möglich. $\mathrm{M}=$ Mittelwert, $\mathrm{SD}=$ Standardabweichung. a 9: 8 Kinder bei NU3. ${ }^{\text {b }}$ Vergleich: Friedman-Test mit Dunns Verteilung. ${ }^{\text {c }}$ Vergleich: Mann-Whitney Test. NU1 $=$ NU2 $=$ NU3 wenn $\mathrm{p}>0,05$ und Zeichen $<$ bzw. $>$ bedeuten $\mathrm{p}<0,05$

\subsubsection{Humerustorsionsdifferenz im Vergleich zu den Röntgenbildern}

Ein seitliches Röntgenbild wurde drei bis vier Wochen (Ø 26 Tage) nach der Operation zur Feststellung der Frakturkonsolidation angefertigt. Hierauf wurde ein potentieller Rotationssporn analysiert und mit ,ja“ oder „,nein“ in den Ergebnissen notiert (Kapitel 2.5). Eine Korrelation des radiologisch identifizierten Humerustorsionsfehlers - gemessen als Rotationssporn bzw. als Achsenfehlstellung - mit der sonographischen Humerustorsionsdifferenz wurde festgestellt (Tabelle 22). Aufgrund der geringen Fallzahl war keine Signifikanztestung möglich. Es zeigte sich, dass Fehlstellungen und Rotationssporne nur bei einer Humerustorsionsdifferenz von $>5^{\circ}$, gemessen bei NU1, auftraten. In der Gruppe retro 1 mit einer Humerustorsionsdifferenz von $>5^{\circ} \mathrm{kam}$ es bei $50 \%$ der Kindern zu Auffälligkeiten (Tabelle 22). In der Gruppe retro 2 mit einer Humerustorsionsdifferenz von $>5^{\circ}$ gab es bei $44 \%$ der Kinder Auffälligkeiten im Röntgen (Tabelle 22). 
Tabelle 22: Ergebnisse der Untersuchung der Humerustorsionsdifferenz im Verhältnis zu den Röntgenaufnahmen (radiologischen Auffälligkeiten wie z. B. Rotationssporn im Seitbild, Achsfehlstellungen): Gruppe retro $1<1$ Jahr nach Unfall; Gruppe retro $2>1$ Jahr nach Unfall

\begin{tabular}{lll} 
Humerustorsionsdifferenz bei NU1 & $\mathrm{n}$ & Röntgen und Anzahl der Kinder \\
\hline $\begin{array}{l}\text { Gruppe retro } 1 \\
\leq 5^{\circ}\end{array}$ & 0 & \\
$>5^{\circ}\left(\varnothing 18,5^{\circ}\right)$ & 10 & Sporn: $2(20 \%)$ \\
& Sporn und Valgus:1 (10\%) \\
& Sporn und Varus: $2(20 \%)$ \\
& Kein Sporn, keine Achsfehlstellung, \\
& kein Bild: $5(50 \%)$
\end{tabular}

\section{Gruppe retro 2}

$\begin{array}{ll}\leq 5^{\circ}\left(\varnothing 4^{\circ}\right) & \begin{array}{l}\text { Kein Sporn, keine Achsfehlstellung, } \\ \text { kein Bild: } 3(100 \%)\end{array} \\ >5^{\circ}\left(\varnothing 21,8^{\circ}\right) & \text { Sporn: } 2(22 \%) \\ & \text { Valgus: } 2(22 \%) \\ & \text { Kein Sporn, keine Achsfehlstellung, } \\ & \text { kein Bild: } 5(56 \%)\end{array}$

Anmerkung. ${ }^{a}$ 9: 8 Kinder bei NU3. / = kein Wert vorhanden

\subsection{Prospektives Kollektiv}

\subsubsection{Beschreibung des prospektiven Kollektivs}

Im prospektiven Kollektiv wurden 27 Kinder in den Jahren zwischen 2002 und 2004 mit suprakondylären Humerusfrakturen im Alter von zwei bis 13 Jahren ( $6,8 \pm 2,6$ Jahre) zum Unfallzeitpunkt (Abbildung 21), nach sechs Wochen, vier Monaten, sechs Monaten und zwölf Monaten nach Unfall untersucht. 


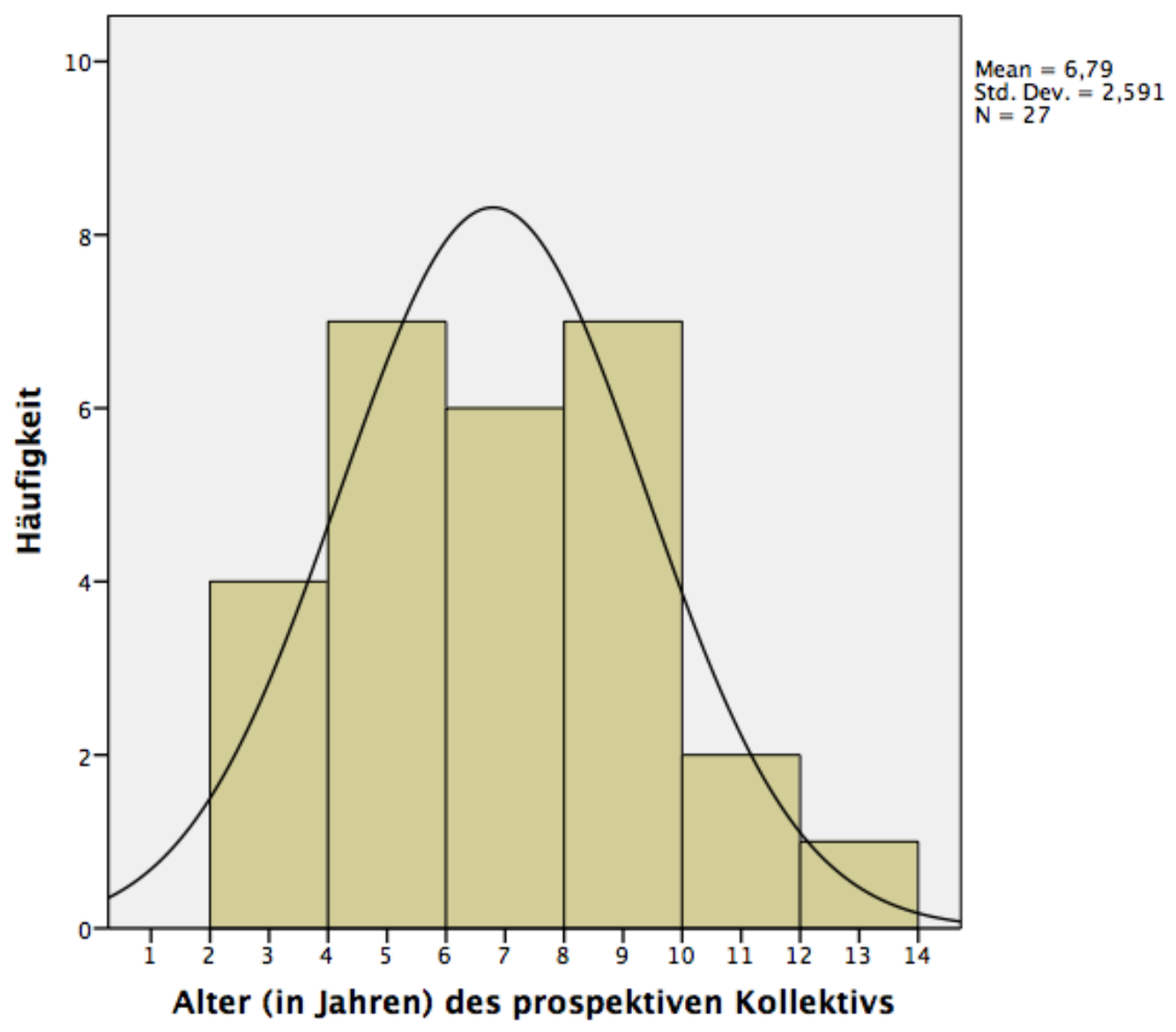

Abbildung 21: Altersverteilung des prospektiven Kollektivs zum Unfallzeitpunkt

Von den 27 Kindern waren 16 Kinder männlich (59,3\%) und elf Kinder weiblich (40,7\%). Bei neun der Kinder (33,3\%) war die rechte und bei 18 (66,6\%) die linke Seite betroffen. 23 der Kinder (85\%) waren Rechtshänder, bei dreien war die Händigkeit beidseits (11\%) und ein Kind (4\%) war Linkshänder. Fast alle Kinder (N=26; 96\%) wurden am Unfalltag operativ versorgt. Ein Patient wurde bei Sekundärdislokation am sechsten Tag nach Unfall operativ versorgt. Bei einem Patienten lag eine temporäre Radialisparese, bei zwei weiteren eine temporäre Parese des N. medianus der frakturierten Extremität als Begleitverletzung vor. Alle Frakturen wurden mittels geschlossener Reposition und perkutan gekreuzter Kirschnerdrahtfixation versorgt (Abbildung 22). Anschließend erfolgte eine Gipsruhigstellung für $\varnothing$ 25,5 Tage (21 bis 37 Tage). Nach Gipsabnahme wurde ein Konsolidationsröntgenbild des Ellenbogens in zwei Ebenen angefertigt und anschließend die gekreuzten K-Drähte ambulant ohne Narkose (N=26) entfernt. Alle Kinder bewegten spontan die verletzte Extremität. Keiner der Patienten erhielt Krankengymnastik.

Als Komplikationen hatten drei Patienten Pin-Infekte, die unter Gabe entsprechender Antibiotika schnell abklangen. Bei einer Patientin wurde eine zweite Operation nach sieben Tagen notwendig, da die Drähte sich gelockert hatten und die ursprüngliche Positionierung nicht mehr gesichert war. Hierdurch kam es zu einer erneuten Dislozierung der Fraktur. Der durchschnittliche Krankenhausaufenthalt lag bei drei Tagen ( $=25)$; zwei Patienten 
waren zehn bzw. zwölf Tage wegen der Pin-Infektionen hospitalisiert. Mit Ausnahme von zwei Patienten erhielten alle Kinder nur eine Narkose. Eine zweite Narkose wurde in einem Fall für einen erneuten Eingriff und im zweiten Fall für eine Metallentfernung von versenkten Drähten notwendig. Es wurden durchschnittlich 2,5 Röntgenkontrolluntersuchungen (Unfallbilder und Konsolidationsröntgen) durchgeführt.
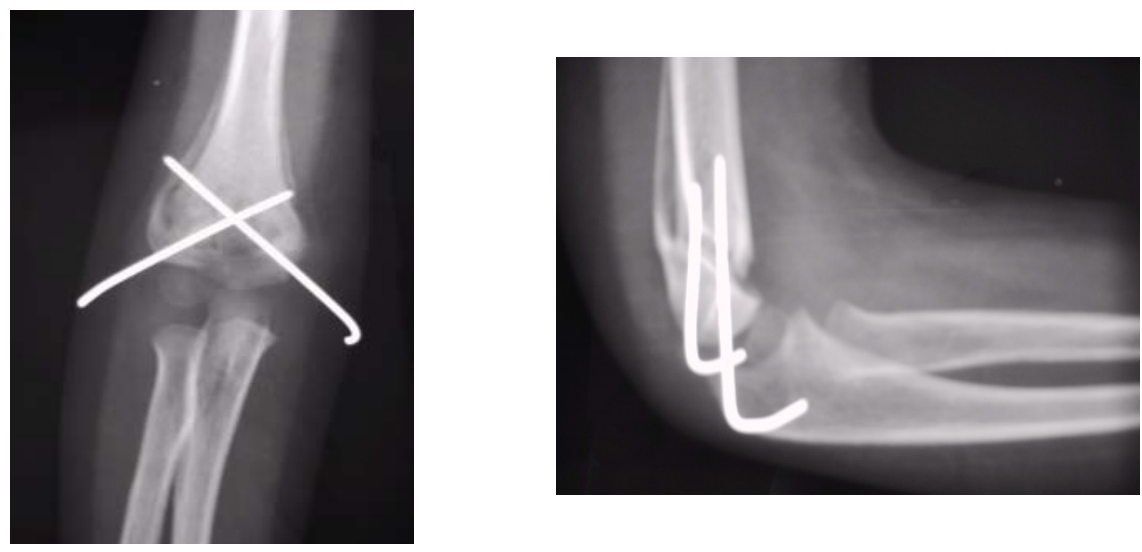

Abbildung 22: Perkutane K-Draht-Osteosynthese von radial und ulnar; links das anteriore-posteriore Röntgenbild, rechts die laterale Aufnahme. Konsolidationsröntgenbild mit Kallusformation drei Wochen postoperativ (Bilder von AK Hell)

\subsubsection{Anamnese und subjektive Beschwerden des prospektiven Kollektivs}

Im Rahmen der Anamnese wurde nach dem Unfallhergang gefragt (Tabelle 23). Hier fanden sich als Hauptursache unbeobachtete Stürze, gefolgt von Stürzen bei sportlichen Aktivitäten.

Tabelle 23: Unfallhergang und Verteilung der Unfallmuster der Kinder des prospektiven Kollektivs $(\mathrm{N}=27)$ bei stattgehabter suprakondylärer Humerusfraktur

\begin{tabular}{lcc}
\hline \multicolumn{1}{c}{ Ätiologie } & Gesamt & Prozent \\
\hline Sturz aus Höhe (Klettergerüst, Baum, Sprossenwand) & 4 & $15 \%$ \\
Unbeobachtete Stürze & 13 & $48 \%$ \\
Sturz von Rutschbahn & 1 & $4 \%$ \\
Sturz bei sportlichen Aktivitäten (Fahrrad fahren, Turnen, Skaten) & 8 & $29 \%$ \\
Banale Stürze (Stolpern, vom Stuhl gefallen) & 1 & $4 \%$ \\
Gesamt & 27 & $100 \%$ \\
\hline
\end{tabular}

Bei jeder NU wurde gefragt, ob kosmetische Beeinträchtigungen, Schmerzen oder Funktionseinschränkungen vorlagen (Tabelle 24). In dieser Tabelle zeigte sich, dass im 
Verlauf der Nachuntersuchungen die Funktionseinschränkungen von 100\% auf $0 \%$ zurückgingen.

Tabelle 24: subjektive Beschwerden im prospektiven Kollektiv $(\mathrm{N}=27)$ zu den jeweiligen Nachuntersuchungsterminen

\begin{tabular}{lcccc}
\hline Subjektive Beschwerden & $\begin{array}{c}\text { Nach 6 } \\
\text { Wochen } \\
(\mathrm{N}=27)\end{array}$ & $\begin{array}{c}\text { Nach 4 } \\
\text { Monaten } \\
(\mathrm{N}=27)\end{array}$ & $\begin{array}{c}\text { Nach 6 } \\
\text { Monaten } \\
(\mathrm{N}=27)\end{array}$ & $\begin{array}{l}\text { Nach 12 } \\
\text { Monaten } \\
(\mathrm{N}=27)\end{array}$ \\
\hline kosmetisch störend & 0 & 0 & 0 & 0 \\
Schmerzen & 0 & 0 & 0 & 0 \\
Funktionseinschränkung & 27 & 5 & 1 & 0 \\
\hline
\end{tabular}

\subsubsection{Klinische Untersuchung des prospektiven Kollektivs}

Auch bei diesem Kollektiv (N=27) wurden zu jedem Nachuntersuchungstermin Innenund Außenrotation der Schulter sowie Extension, Flexion, Pronation und Supination des Ellenbogens und der Achsenstatus des jeweiligen gesunden und frakturierten Armes untersucht (Abbildung 15 und Kapitel 2.5).

\subsubsection{Innenrotation der Schulter im prospektiven Kollektiv}

Bei der klinischen Untersuchung ergab sich beim Vergleich der Innenrotation zwischen der gesunden und der ehemals frakturierten Seite nur nach zwölf Monaten nach Unfall ein signifikanter Unterschied ( $\mathrm{p}<0,05)$, wobei sich hier die absoluten Mittelwerte nur marginal unterschieden (Tabelle 25). 
Tabelle 25: Ergebnisse der Untersuchung der Schulterinnenrotationen im prospektiven Kollektiv N=27

\begin{tabular}{|c|c|c|c|c|}
\hline \multirow{3}{*}{$\begin{array}{l}\text { Untersuchungs- } \\
\text { zeitpunkt }\end{array}$} & \multicolumn{3}{|c|}{ Vergleich der gesunden mit der frakturierten Seite } & \multirow[b]{2}{*}{$\begin{array}{l}\text { Differenz frakturierte- } \\
\text { gesunde Seite }\end{array}$} \\
\hline & $\begin{array}{l}\text { Gesunde } \\
\text { Seite }\end{array}$ & $\begin{array}{l}\text { Frakturierte } \\
\text { Seite }\end{array}$ & & \\
\hline & $\mathrm{M} \pm 1 \mathrm{SD}$ & $\mathrm{M} \pm 1 \mathrm{SD}$ & $\mathrm{p}$-Wert ${ }^{\mathrm{b}}$ & $\mathrm{M} \pm 1 \mathrm{SD}$ \\
\hline 6 Wochen $(6 \mathrm{~W})$ & $76,1 \pm 10,6^{\circ}$ & $79,3 \pm 11,4^{\circ}$ & $p>0,05$ & $7,5 \pm 9,3^{\circ}$ \\
\hline 4 Monate $(4 \mathrm{M})$ & $76,7 \pm 10,4^{\circ}$ & $78,2 \pm 11,1^{\circ}$ & $\mathrm{p}>0,05$ & $4,6 \pm 7,1^{\circ}$ \\
\hline 6 Monate $(6 \mathrm{M})^{\mathrm{a}}$ & $77,1 \pm 9,4^{\circ}$ & $79,6 \pm 10,4^{\circ}$ & $\mathrm{p}>0,05$ & $5,0 \pm 5,8^{\circ}$ \\
\hline 12 Monate $(12 \mathrm{M})$ & $76,3 \pm 10,1^{\circ}$ & $80,9 \pm 8,8^{\circ}$ & $\mathrm{p}<0,05$ & $7,3 \pm 7,8^{\circ}$ \\
\hline \multirow[t]{2}{*}{ Vergleich ${ }^{c}$} & $6 \mathrm{~W}=4 \mathrm{M}=$ & $6 \mathrm{~W}=4 \mathrm{M}=$ & & $6 \mathrm{~W}=4 \mathrm{M}=$ \\
\hline & $6 \mathrm{M}=12 \mathrm{M}$ & $6 \mathrm{M}=12 \mathrm{M}$ & & $6 \mathrm{M}=12 \mathrm{M}$ \\
\hline
\end{tabular}

Anmerkung. $\mathrm{M}=$ Mittelwert. $\mathrm{SD}=$ Standardabweichung.

${ }^{\mathrm{a}} 6$ Monate $(6 \mathrm{M}): \mathrm{N}=26 .{ }^{\mathrm{b}} \mathrm{p}$-Wert: Paired t-Test außer nach 6 Monaten: Wilcoxon matched pairs Test. " Vergleich: frakturierte Seite repeated measures ANOV A mit Bonferroni Verteilung. Gesunde Seite und Differenz frakturierte- gesunde Seite: Friedman-Test mit Dunns Verteilung. $6 \mathrm{~W}=4 \mathrm{M}=6 \mathrm{M}=12 \mathrm{M}$ wenn $\mathrm{p}>0,05$

\subsubsection{Außenrotation der Schulter im prospektiven Kollektiv}

Bei der Schulteraußenrotation zeigte sich nach sechs Wochen, vier Monaten und zwölf Monaten ein signifikanter Unterschied $(p<0,05)$ zwischen der gesunden und der frakturierten oberen Extremität. Im Verlauf der Nachuntersuchungen war eine Zunahme der Beweglichkeit hinsichtlich der Schulteraußenrotation zu erkennen (Tabelle 26). Zwischen der gesunden und frakturierten Seite ergaben sich nach sechs Wochen, vier- und zwölf Monaten signifikante Unterschiede. 
Tabelle 26: Ergebnisse der Untersuchung der Schulteraußenrotation im prospektiven Kollektiv N=27

\begin{tabular}{|c|c|c|c|c|}
\hline \multirow{4}{*}{$\begin{array}{l}\text { Untersuchungs- } \\
\text { zeitpunkt }\end{array}$} & \multicolumn{3}{|c|}{ Vergleich der gesunden mit der frakturierten Seite } & \multirow{3}{*}{$\begin{array}{l}\text { Differenz frakturierte- } \\
\text { gesunde Seite }\end{array}$} \\
\hline & & Frakturierte & & \\
\hline & Gesunde Seite & Seite & & \\
\hline & $\mathrm{M} \pm 1 \mathrm{SD}$ & $\mathrm{M} \pm 1 \mathrm{SD}$ & $\mathrm{p}-$ Wert $^{\mathrm{b}}$ & $\mathrm{M} \pm 1 \mathrm{SD}$ \\
\hline 6 Wochen $(6 \mathrm{~W})$ & $105,0 \pm 12,0^{\circ}$ & $98,5 \pm 11,6^{\circ}$ & $\mathrm{p}<0,05$ & $8,3 \pm 9,8^{\circ}$ \\
\hline 4 Monate $(4 \mathrm{M})$ & $104,4 \pm 11,2^{\circ}$ & $100,4 \pm 12,2^{\circ}$ & $\mathrm{p}<0,05$ & $6,5 \pm 8,5^{\circ}$ \\
\hline 6 Monate $(6 \mathrm{M})^{a}$ & $105,6 \pm 12,2^{\circ}$ & $102,9 \pm 11,3^{\circ}$ & $\mathrm{p}>0,05$ & $5,4 \pm 7,6^{\circ}$ \\
\hline 12 Monate $(12 \mathrm{M})$ & $105,7 \pm 11,3^{\circ}$ & $101,3 \pm 11,7^{\circ}$ & $\mathrm{p}<0,05$ & $5,8 \pm 7,5^{\circ}$ \\
\hline \multirow[t]{2}{*}{ Vergleich ${ }^{c}$} & $6 \mathrm{~W}=4 \mathrm{M}=$ & $6 \mathrm{~W}=4 \mathrm{M}=$ & & $6 \mathrm{~W}=4 \mathrm{M}=$ \\
\hline & $6 \mathrm{M}=12 \mathrm{M}$ & $6 \mathrm{M}=12 \mathrm{M}$ & & $6 \mathrm{M}=12 \mathrm{M}$ \\
\hline
\end{tabular}

Anmerkung. $\mathrm{M}=$ Mittelwert. $\mathrm{SD}=$ Standardabweichung.

a 6 Monate $(6 \mathrm{M}): \mathrm{N}=26 .{ }^{\mathrm{b}} \mathrm{p}$-Wert: Wilcoxon matched pairs Test“. c Vergleich: Friedman-

Test mit Dunns Verteilung. $6 \mathrm{~W}=4 \mathrm{M}=6 \mathrm{M}=12 \mathrm{M}$ wenn $\mathrm{p}>0,05$

\subsubsection{Ellenbogenextension im prospektiven Kollektiv}

Die Ellenbogenextension war nach sechs Wochen sowie nach vier- und sechs Monaten an der erkrankten oberen Extremität im Gegensatz zur gesunden oberen Extremität signifikant eingeschränkt (Tabelle 27).

Während bei den Nachuntersuchungen nach sechs Wochen und vier Monaten die Kinder noch Bewegungseinschränkungen aufwiesen (in der Tabelle 27 in der Spalte „Frakturierte Seite“ als Plus-Werte dargestellt), waren diese in den nachfolgenden Nachuntersuchungen (nach sechs- und zwölf Monaten) nicht mehr vorhanden. Die Anzahl der Kinder mit Bewegungseinschränkungen nahm also im Verlaufe der Nachuntersuchungen deutlich ab (nach sechs Wochen noch 24 Kinder, nach vier Monaten neun Kinder, nach sechs Monaten drei Kinder, nach zwölf Monaten nur ein Kind).

Auf der gesunden Seite ergaben sich bezüglich des Mittelwertes keine signifikanten Unterschiede zu den verschiedenen Untersuchungszeitpunkten. Auf der frakturierten Seite und bei der Differenzbildung zwischen gesundem und frakturiertem Arm fanden sich jedoch signifikante Unterschiede zwischen den Untersuchungszeitpunkten nach sechs Wochen versus vier Monate, sechs Wochen versus sechs Monate, sechs Wochen und zwölf Monate sowie vier und zwölf Monate. 
Zusammenfassend besserte sich das Ellenbogenextensionsdefizit signifikant im Verlauf der Untersuchungszeiträume und zeigte nach zwölf Monaten nur noch einen marginalen Unterschied zum gesunden Arm (trotz vorhandener Signifikanz).

Tabelle 27: Ergebnisse der Untersuchung der Ellenbogenextension im prospektiven Kollektiv mit $\mathrm{N}=27$

Vergleich der gesunden mit der frakturierten Seite

\begin{tabular}{|c|c|c|c|c|}
\hline $\begin{array}{l}\text { Untersuchungs- } \\
\text { zeitpunkt }\end{array}$ & $\begin{array}{l}\text { Gesunde Seite } \\
\qquad \pm 1 \text { SD }\end{array}$ & $\begin{array}{l}\text { Frakturierte Seite } \\
\qquad \mathrm{M} \pm 1 \mathrm{SD}\end{array}$ & $\mathrm{p}-$ Wert $^{\mathrm{b}}$ & $\begin{array}{l}\text { Differenz frakturierte- } \\
\text { gesunde Seite } \\
\mathrm{M} \pm 1 \mathrm{SD}\end{array}$ \\
\hline 6 Wochen $(6 \mathrm{~W})$ & $-11,9 \pm 4,2^{\circ}$ & $28,3 \pm 20,9^{\circ}$ & $\mathrm{p}<0,05$ & $40,8 \pm 19,9^{\circ}$ \\
\hline 4 Monate (4M) & $-12,6 \pm 4,0^{\circ}$ & $1,5 \pm 12,2^{\circ}$ & $\mathrm{p}<0,05$ & $12,9 \pm 11,4^{\circ}$ \\
\hline 6 Monate $(6 \mathrm{M})^{\mathrm{a}}$ & $-11,9 \pm 3,8^{\circ}$ & $-5,8 \pm 9,3^{\circ}$ & $\mathrm{p}<0,05$ & $5,2 \pm 9,1^{\circ}$ \\
\hline 12 Monate $(12 \mathrm{M})$ & $-12,4 \pm 3,2^{\circ}$ & $-10,6 \pm 4,7^{\circ}$ & $\mathrm{p}<0,05$ & $0,8 \pm 3,9^{\circ}$ \\
\hline \multirow[t]{4}{*}{ Vergleich ${ }^{c}$} & $6 \mathrm{~W}=4 \mathrm{M}=$ & $6 \mathrm{~W}>4 \mathrm{M}$ & & $6 \mathrm{~W}>4 \mathrm{M}$ \\
\hline & $6 \mathrm{M}=12 \mathrm{M}$ & $6 \mathrm{~W}>6 \mathrm{M}$ & & $6 \mathrm{~W}>6 \mathrm{M}$ \\
\hline & & $6 \mathrm{~W}>12 \mathrm{M}$ & & $6 \mathrm{~W}>12 \mathrm{M}$ \\
\hline & & $4 M>12 M$ & & $4 M>12 M$ \\
\hline
\end{tabular}

Anmerkung. $\mathrm{M}=$ Mittelwert. $\mathrm{SD}=$ Standardabweichung. Die Minuswerte bedeuten eine Hyperextension des Ellenbogens.

a 6 Monate $(6 \mathrm{M}): \mathrm{N}=26 .{ }^{\mathrm{b}} \mathrm{p}$-Wert: Wilcoxon matched pairs Test. c Vergleich: Friedman-Test mit Dunns Verteilung“" $6 \mathrm{~W}=4 \mathrm{M}=6 \mathrm{M}=12 \mathrm{M}$ wenn $\mathrm{p}>0,05$ und Zeichen $<$ bzw. $>$ bedeuten $\mathrm{p}<0,05$

\subsubsection{Ellenbogenflexion im prospektiven Kollektiv}

Bei der Ellenbogenflexion zeigte sich nach sechs Wochen, vier- und sechs Monaten ein signifikanter Unterschied zwischen den beiden oberen Extremitäten. Nach zwölf Monaten ergab sich kein signifikanter Unterschied mehr ( $p>0,05$; Tabelle 28). Die Ellenbogenflexion auf der frakturierten Seite war zwischen sechs Wochen und vier Monaten, sechs Wochen und sechs Monaten, sechs Wochen und zwölf Monaten sowie vier Monaten und zwölf Monaten signifikant unterschiedlich. Wurde auch hier die Differenz gebildet zwischen dem gesunden und frakturierten Arm jedes Kindes in den einzelnen Nachuntersuchungen, so zeigten sich Signifikanzen bei der Differenzbildung zwischen sechs Wochen und vier Monate, sechs Wochen und sechs Monate sowie zwischen sechs Wochen und zwölf Monate. 
Zusammenfassend besserte sich die Ellenbogenflexion signifikant im Verlauf der Untersuchungszeiträume und zeigte nach zwölf Monaten nur noch einen marginalen, nicht signifikanten, Unterschied zum gesunden Arm.

Tabelle 28: Ergebnisse der Untersuchung der Ellenbogenflexion im prospektiven Kollektiv mit $\mathrm{N}=27$

\begin{tabular}{|c|c|c|c|c|}
\hline \multirow{3}{*}{$\begin{array}{l}\text { Untersuchungs- } \\
\text { zeitpunkt }\end{array}$} & \multicolumn{3}{|c|}{ Vergleich der gesunden mit der frakturierten Seite } & \multirow{3}{*}{$\begin{array}{l}\text { Differenz frakturierte-gesunde } \\
\text { Seite } \mathrm{M} \pm 1 \mathrm{SD}\end{array}$} \\
\hline & $\begin{array}{l}\text { Gesunde } \\
\text { Seite }\end{array}$ & $\begin{array}{l}\text { Frakturierte } \\
\text { Seite }\end{array}$ & & \\
\hline & $\mathrm{M} \pm 1 \mathrm{SD}$ & $\mathrm{M} \pm 1 \mathrm{SD}$ & $\mathrm{p}-$ Wert $^{\mathrm{b}}$ & \\
\hline 6 Wochen $(6 \mathrm{~W})$ & $140,9 \pm 4,6^{\circ}$ & $126,9 \pm 10,2^{\circ}$ & $\mathrm{p}<0,05$ & $14,0 \pm 9,1^{\circ}$ \\
\hline 4 Monate $(4 \mathrm{M})$ & $140,7 \pm 3,6^{\circ}$ & $133,3 \pm 9,2^{\circ}$ & $\mathrm{p}<0,05$ & $6,5 \pm 11,3^{\circ}$ \\
\hline 6 Monate $(6 \mathrm{M})^{\mathrm{a}}$ & $140,8 \pm 3,7^{\circ}$ & $137,1 \pm 7,4^{\circ}$ & $\mathrm{p}<0,05$ & $2,9 \pm 6,7^{\circ}$ \\
\hline 12 Monate $(12 \mathrm{M})$ & $141,7 \pm 3,9^{\circ}$ & $140,9 \pm 4,4^{\circ}$ & $\mathrm{p}>0,05$ & $0,4 \pm 2,0^{\circ}$ \\
\hline Vergleich ${ }^{c}$ & $\begin{array}{l}6 \mathrm{~W}=4 \mathrm{M}= \\
6 \mathrm{M}=12 \mathrm{M}\end{array}$ & $\begin{array}{c}6 \mathrm{~W}<4 \mathrm{M} \\
6 \mathrm{~W}<6 \mathrm{M} \\
6 \mathrm{~W}<12 \mathrm{M} \\
4 \mathrm{M}<12 \mathrm{M}\end{array}$ & & $6 \mathrm{~W}>4 \mathrm{M}=6 \mathrm{M}=12 \mathrm{M}$ \\
\hline
\end{tabular}

Anmerkung. $\mathrm{M}=$ Mittelwert. $\mathrm{SD}=$ Standardabweichung.

${ }^{a} 6$ Monate (6M): $\mathrm{N}=26 .{ }^{\mathrm{b}}$ p-Wert: Wilcoxon matched pairs Test. ${ }^{c}$ Vergleich: Friedman-Test mit Dunns Verteilung. 6W=4M=6M=12M wenn $\mathrm{p}>0,05$ und Zeichen $<$ bzw. $>$ bedeuten $\mathrm{p}<0,05$

\subsubsection{Pronation und Supination des Unterarmes im prospektiven Kollektiv}

Die Pronation blieb bei den gesamten Nachuntersuchungen bei allen Kindern bei $90^{\circ}$ und zeigte somit keine Änderungen.

Nur bei zwei von den 27 Kindern bei der Nachuntersuchung nach sechs Wochen zeigten sich Einschränkungen in der Supination. Diese waren im Vergleich zur gesunden Extremität nicht signifikant unterschiedlich. Bei allen nachfolgenden Untersuchungen zeigte sich auf beiden oberen Extremitäten eine identische Supination von $90^{\circ}$.

\subsubsection{Ellenbogenachse des prospektiven Kollektivs}

Die Ellenbogenachse wurde nur bei freier Extension bestimmt. Beim Termin nach sechs Wochen war diese in 21 Fällen noch nicht wiederhergestellt, sodass dieser Zeitraum für 
diese Untersuchung nicht berücksichtigt wurde. Bei der Untersuchung nach vier Monaten war die freie Extension bei sechs Kindern und bei der Untersuchung nach sechs Monaten bei einem Kind noch nicht möglich. Ein anderes Kind war zur NU nach sechs Monaten nicht erschienen. Für diese Kinder konnten keine Daten erhoben werden. Um die Daten besser miteinander vergleichen zu können, wurden nur die Kinder berücksichtigt, die in allen Nachuntersuchungen eine bestimmbare Ellenbogenachse hatten. Das war dann bei 20 Kindern bei der NU nach vier Monaten der Fall (Tabelle 29).

Zwischen der gesunden und frakturierten Seite bestand nur nach sechs Monaten ein signifikanter Unterschied. Weitere signifikante Unterschiede fanden sich nicht.

Tabelle 29: Ergebnisse der Untersuchung der Mittelwerte der absoluten klinischen Achsmessung am Arm im prospektiven Kollektiv, beginnend nach 4 Monaten nach Unfall, da hier bei 20 Kindern die Ellenbogenachse wiederhergestellt war

\begin{tabular}{|c|c|c|c|c|}
\hline \multirow[b]{3}{*}{$\begin{array}{l}\text { Untersuchungs- } \\
\text { zeitpunkt }\end{array}$} & \multicolumn{3}{|c|}{ Vergleich der gesunden mit der frakturierten Seite } & \multirow[b]{3}{*}{$\begin{array}{l}\text { Differenz frakturierte- } \\
\text { gesunde Seite } \mathrm{M} \pm 1 \mathrm{SD}\end{array}$} \\
\hline & & Frakturierte & & \\
\hline & $\begin{array}{c}\text { Gesunde Seite } \\
\mathrm{M} \pm 1 \mathrm{SD}\end{array}$ & $\begin{array}{c}\text { Seite } \\
\mathrm{M} \pm 1 \mathrm{SD}\end{array}$ & $\mathrm{p}-$ Werta & \\
\hline 4 Monate $(4 \mathrm{M})$ & $-8,5 \pm 2,9^{\circ}$ & $-7,5 \pm 4,1^{\circ}$ & $\mathrm{p}>0,05$ & $1,5 \pm 2,6^{\circ}$ \\
\hline 6 Monate $(6 \mathrm{M})$ & $-8,5 \pm 3,3^{\circ}$ & $-6,5 \pm 4,6^{\circ}$ & $\mathrm{p}<0,05$ & $2,0 \pm 3,4^{\circ}$ \\
\hline 12 Monate (12M) & $-8,5 \pm 3,7^{\circ}$ & $-6,5 \pm 5,6^{\circ}$ & $\mathrm{p}>0,05$ & $1,5 \pm 2,9^{\circ}$ \\
\hline Vergleichb & $4 \mathrm{M}=6 \mathrm{M}=12 \mathrm{M}$ & $4 \mathrm{M}=6 \mathrm{M}=12 \mathrm{M}$ & & $4 \mathrm{M}=6 \mathrm{M}=12 \mathrm{M}$ \\
\hline
\end{tabular}

Anmerkung. $\mathrm{M}=$ Mittelwert. $\mathrm{SD}=$ Standardabweichung. - = Cubitus valgus. ${ }^{a}$ p-Wert: Paired t-Test, außer nach 4 und 12 Monaten: Wilcoxon matched pairs Test. b Vergleich: Friedman-Test mit Dunns Verteilung. 4M=6M=12M wenn $p>0,05$ und Zeichen $<$ bzw. $>$ bedeuten $\mathrm{p}<0,05$

\subsubsection{Sonographische Humerustorsionsmessung im prospektiven Kollektiv}

Bei der sonographischen Untersuchung der Humerustorsion (Kapitel 2.5.3) im prospektiven Kollektiv ( $\mathrm{N}=27)$ zeigte sich ein signifikanter Unterschied bei allen Nachuntersuchungen zwischen dem Frakturarm und der gesunden Extremität. Diese Differenz war am größten sechs Wochen nach Fraktur (Tabelle 30). Es ergaben sich bei Analyse der Differenzbildung signifikante Unterschiede zwischen sechs Wochen und sechs Monate, sechs Wochen und zwölf Monate sowie zwischen vier Monate und zwölf Monate. Die Differenzwerte wurden im Verlauf immer niedriger. Bereits zwischen sechs 
Wochen und vier Monate kam es zu einer deutlichen, jedoch nicht signifikanten Abnahme. Der erste signifikante Unterschied fand sich zwischen sechs Wochen und sechs Monate. Nach außen gerichtete sonographisch gemessene Torsionen (lateral) gingen als negative Zahl in die Berechnung ein und nach innen (medial) gerichtete Torsionen als positive.

Tabelle 30: Ergebnisse der Untersuchung der sonographisch gemessenen Humerustorsionswerte bei Kindern nach suprakondylärer Humerusfraktur im prospektiven Kollektiv (N=27)

Vergleich der gesunden mit der frakturierten Seite

Differenz frakturierte-

\begin{tabular}{|c|c|c|c|c|}
\hline $\begin{array}{l}\text { Untersuchungs- } \\
\text { zeitpunkt }\end{array}$ & $\begin{array}{c}\text { Gesunde Seite } \\
\mathrm{M} \pm 1 \mathrm{SD}\end{array}$ & $\begin{array}{l}\text { Frakturierte Seite } \\
\qquad \mathrm{M} \pm 1 \mathrm{SD}\end{array}$ & $\mathrm{p}$-Wert ${ }^{\mathrm{b}}$ & $\begin{array}{l}\text { gesunde Seite } \\
\mathrm{M} \pm 1 \mathrm{SD}\end{array}$ \\
\hline 6 Wochen (6W) & $20,9 \pm 14,8^{\circ}$ & $28,4 \pm 11,0^{\circ}$ & $\mathrm{p}<0,05$ & $9,8 \pm 8,7^{\circ}$ \\
\hline 4 Monate $(4 \mathrm{M})$ & $22,3 \pm 13,7^{\circ}$ & $27,4 \pm 10,0^{\circ}$ & $\mathrm{p}<0,05$ & $6,2 \pm 7,2^{\circ}$ \\
\hline 6 Monate $(6 \mathrm{M})^{\mathrm{a}}$ & $23,7 \pm 12,7^{\circ}$ & $27,7 \pm 9,2^{\circ}$ & $\mathrm{p}<0,05$ & $5,5 \pm 6,3^{\circ}$ \\
\hline 12 Monate $(12 \mathrm{M})$ & $24,9 \pm 12,2^{\circ}$ & $28,1 \pm 9,5^{\circ}$ & $\mathrm{p}<0,05$ & $3,9 \pm 5,6^{\circ}$ \\
\hline Vergleich ${ }^{c}$ & $\begin{array}{c}6 \mathrm{~W}>6 \mathrm{M}=12 \mathrm{M} \\
4 \mathrm{M}>12 \mathrm{M} \\
6 \mathrm{~W}=4 \mathrm{M}\end{array}$ & $6 \mathrm{M}=4 \mathrm{M}=6 \mathrm{M}=12 \mathrm{M}$ & & $\begin{array}{c}6 \mathrm{~W}>6 \mathrm{M}=12 \mathrm{M} \\
4 \mathrm{M}>12 \mathrm{M} \\
6 \mathrm{~W}=4 \mathrm{M}\end{array}$ \\
\hline
\end{tabular}

Anmerkung $\mathrm{M}=$ Mittelwert. $\mathrm{SD}=$ Standardabweichung.

a 6 Monate (6M): $\mathrm{N}=26 .{ }^{\mathrm{b}} \mathrm{p}$-Wert: Paired t-Test. c Vergleich: repeated measures ANOVA mit Bonferroni Verteilung, außer bei der Differenz: Friedman-Test mit Dunns Verteilung. $6 \mathrm{~W}=4 \mathrm{M}=6 \mathrm{M}=12 \mathrm{M}$ wenn $\mathrm{p}>0,05$ und Zeichen $<$ bzw. $>$ bedeuten $\mathrm{p}<0,05$

Wurde eine sonographisch gemessene Humerustorsionsdifferenz von $<5^{\circ}$ als kein Unterschied gewertet, fand sich folgende Situation:

Im untersuchten prospektiven Kollektiv $(\mathrm{N}=27$ ) hatten sechs Wochen nach Unfall 67\% $(n=18)$ eine sonographisch gemessene Humerustorsionsdifferenz

Ein Kind entwickelte einmalig in der Gruppe „keine Humerustorsionsdifferenz“ ( $n=9)$ nach sechs Monaten eine Humerustorsionsdifferenz von $9{ }^{\circ}$. In der Gruppe mit vorhandener Humerustorsionsdifferenz fand bei elf Kindern (61\%) bis zur NU nach zwölf Monaten eine Detorsion auf $\leq 5^{\circ}$ statt. 
In der Gruppe mit der Humerustorsionsdifferenz ( $\mathrm{n}=18)$ kam es zu signifikanten Unterschieden zwischen sechs Wochen und vier Monate, sechs Wochen und sechs Monate sowie zwischen sechs Wochen und zwölf Monate. Weiterhin ergaben sich für die Zeiträume sechs Wochen und vier Monate signifikante Unterschiede zwischen Kindern ohne und mit vorhandener Humerustorsionsdifferenz. Die Tabelle 31 zeigt, dass die Größe der Humerustorsionsdifferenz in beiden Gruppen nach zwölf Monaten reduziert werden konnte.

Tabelle 31: Ergebnisse der Untersuchung der sonographisch gemessenen Humerustorsionsdifferenz bei Kindern nach suprakondylärer Humerusfraktur im prospektiven Kollektiv $(\mathrm{N}=27)$

\begin{tabular}{|c|c|c|c|c|c|}
\hline & $\begin{array}{l}6 \text { Wochen } \\
(6 \mathrm{~W})\end{array}$ & $\begin{array}{l}4 \text { Monate } \\
\qquad(4 \mathrm{M})\end{array}$ & $\begin{array}{l}6 \text { Monate } \\
(6 \mathrm{M})\end{array}$ & $\begin{array}{c}12 \text { Monate } \\
(12 \mathrm{M})\end{array}$ & $\begin{array}{c}\text { Vergleich je } \\
\text { NUb }^{\mathrm{b}}\end{array}$ \\
\hline Gruppenaufteilung & $\mathrm{M} \pm 1 \mathrm{SD}$ & $M \pm 1 S D$ & $\mathrm{M} \pm 1 \mathrm{SD}$ & $\mathrm{M} \pm 1 \mathrm{SD}$ & \\
\hline $\begin{array}{l}\text { Keine Humerustorsions- } \\
\text { differenz } \\
\left(\leq 5^{\circ} ; n=9 ; 33 \%\right)\end{array}$ & $1,4 \pm 1,9^{\circ}$ & $1,1 \pm 1,7^{\circ}$ & $2,4 \pm 2,9^{\circ}$ & $0,8 \pm 1,4^{\circ}$ & $\begin{array}{l}6 \mathrm{~W}=4 \mathrm{M}= \\
6 \mathrm{M}=12 \mathrm{M}\end{array}$ \\
\hline $\begin{array}{l}\text { Humerustorsionsdifferenz } \\
\left(>5^{\circ} ; \varnothing 14,0^{\circ} ; \mathrm{n}=18^{\mathrm{a}} \text {; }\right. \\
67 \%)\end{array}$ & $14,0 \pm 7,6^{\circ}$ & $7,8 \pm 8,4^{\circ}$ & $6,5 \pm 7,6^{\circ}$ & $4,9 \pm 6,6^{\circ}$ & $\begin{array}{l}6 \mathrm{~W}>4 \mathrm{M}=6 \mathrm{M} \\
=12 \mathrm{M}\end{array}$ \\
\hline $\mathrm{p}$-Wertc & $\mathrm{p}<0,05$ & $\mathrm{p}<0,05$ & $p>0,05$ & $\mathrm{p}>0,05$ & \\
\hline
\end{tabular}

Anmerkung. $\mathrm{M}=$ Mittelwert. $\mathrm{SD}=$ Standardabweichung.

${ }^{a} \mathrm{n}=18$ : nach 6 Monaten $\mathrm{n}=17$. ${ }^{\mathrm{b}}$ Vergleich je NU: Keine Humerustorsionsdifferenz: repeated measures ANOV A mit Bonferroni Verteilung. Bei Humerustorsionsdifferenz:

Friedman-Test mit Dunns Verteilung. ${ }^{c}$ p-Wert: Unpaired t-Test bis auf NU 12 Monate: Mann-Whitney Test. $6 \mathrm{~W}=4 \mathrm{M}=6 \mathrm{M}=12 \mathrm{M}$ wenn $\mathrm{p}>0,05$ und Zeichen $<$ bzw. $>$ bedeuten $\mathrm{p}<0,05$

In der Tabelle 32 wurde nur das Kollektiv analysiert, welches eine vorhandene Humerustorsionsdifferenz von $>5^{\circ}$ hatte. Hier fanden sich zwischen allen Nachuntersuchungszeitpunkten signifikante Unterschiede zwischen der gesunden und frakturierten Seite. Bei der Differenzbildung kam es zu signifikanten Unterschieden zwischen sechs Wochen und vier Monate, sechs Wochen und sechs Monate, sechs Wochen und zwölf Monate sowie zwischen vier und zwölf Monate. 
Tabelle 32: Ergebnisse der Untersuchung der sonographisch gemessenen Humerustorsionswerte bei Kindern mit sonographisch gemessenen Differenzen zwischen beiden Extremitäten $>5^{\circ}(\mathrm{N}=18)$

Vergleich der gesunden mit der frakturierten Seite

\begin{tabular}{|c|c|c|c|c|}
\hline $\begin{array}{l}\text { Untersuchungs- } \\
\text { zeitpunkt }\end{array}$ & $\begin{array}{c}\text { Gesunde Seite } \\
\mathrm{M} \pm 1 \mathrm{SD}\end{array}$ & $\begin{array}{l}\text { Frakturierte Seite } \\
\qquad \mathrm{M} \pm 1 \mathrm{SD}\end{array}$ & $\mathrm{p}$-Wert ${ }^{\mathrm{b}}$ & $\begin{array}{l}\text { Differenz frakturierte- } \\
\text { gesunde Seite } \\
\qquad \pm 1 \text { SD }\end{array}$ \\
\hline 6 Wochen $(6 \mathrm{~W})$ & $16,5 \pm 15,6^{\circ}$ & $28,5 \pm 12,5^{\circ}$ & $\mathrm{p}<0,05$ & $14,0 \pm 7,6^{\circ}$ \\
\hline 4 Monate (4M) & $19,1 \pm 15,0^{\circ}$ & $26,9 \pm 10,9^{\circ}$ & $\mathrm{p}<0,05$ & $8,8 \pm 7,6^{\circ}$ \\
\hline 6 Monate $(6 M)^{a}$ & $21,3 \pm 13,8^{\circ}$ & $27,2 \pm 9,4^{\circ}$ & $\mathrm{p}<0,05$ & $7,1 \pm 7,1^{\circ}$ \\
\hline 12 Monate $(12 \mathrm{M})$ & $22,7 \pm 12,9^{\circ}$ & $27,4 \pm 9,8^{\circ}$ & $\mathrm{p}<0,05$ & $5,4 \pm 6,3^{\circ}$ \\
\hline Vergleichc & $\begin{array}{c}6 \mathrm{~W}>6 \mathrm{M}=12 \mathrm{M} \\
4 \mathrm{M}>12 \mathrm{M} \\
6 \mathrm{~W}=4 \mathrm{M}\end{array}$ & $6 \mathrm{M}=4 \mathrm{M}=6 \mathrm{M}=12 \mathrm{M}$ & & $\begin{array}{l}6 W>4 M \\
6 W>6 M \\
6 W>12 M \\
4 M>12 M\end{array}$ \\
\hline
\end{tabular}

Anmerkung $\mathrm{M}=$ Mittelwert. $\mathrm{SD}=$ Standardabweichung.

a 6 Monate $(6 \mathrm{M}): \mathrm{N}=17 .{ }^{\mathrm{b}} \mathrm{p}$-Wert: Paired t-Test. c Vergleich: repeated measures ANOVA mit Bonferroni Verteilung. $6 \mathrm{~W}=4 \mathrm{M}=6 \mathrm{M}=12 \mathrm{M}$ wenn $\mathrm{p}>0,05$ und Zeichen $<$ bzw. $>$ bedeuten $\mathrm{p}<0,05$

\subsubsection{Humerustorsionsänderung im prospektiven Kollektiv}

\subsubsection{Humerustorsionsdifferenz in Abhängigkeit von Ausmaß, Richtung und Alter in den nachfolgenden Untersuchungen}

Analog zur Untersuchung zum retrospektiven Kollektiv wurden drei Altersgruppen gebildet: < 5-Jährige, 5- bis 10-Jährige und > 10-Jährige. Diese Altersgruppen wurden in Abhängigkeit zur Humerustorsionsdifferenz und Richtung analysiert.

Anhand der Daten, die sich auf sehr kleine Kollektive bezogen, kam es zu den größten sonographisch gemessenen Detorsionen, wenn die Humerusinnentorsion bei $\geq 20^{\circ}$ lag. Signifikante Unterschiede gab es zwischen der Außentorsion und der geringen Innentorsion sowie zwischen der Außentorsion und der erhöhten Innentorsion. Bei sehr kleinen Fallzahlen zeigten Kinder, die $<$ zehn Jahre alt waren mit einer vermehrten Außentorsion und erhöhten Innentorsion die besten Korrekturen (Tabelle 33). 
Tabelle 33: Humerustorsionsdifferenzänderung in Abhängigkeit vom Ausmaß der primären Torsionsdifferenz bei der NU nach 6 Wochen und in Abhängigkeit vom Alter 6 Wochen nach Unfall

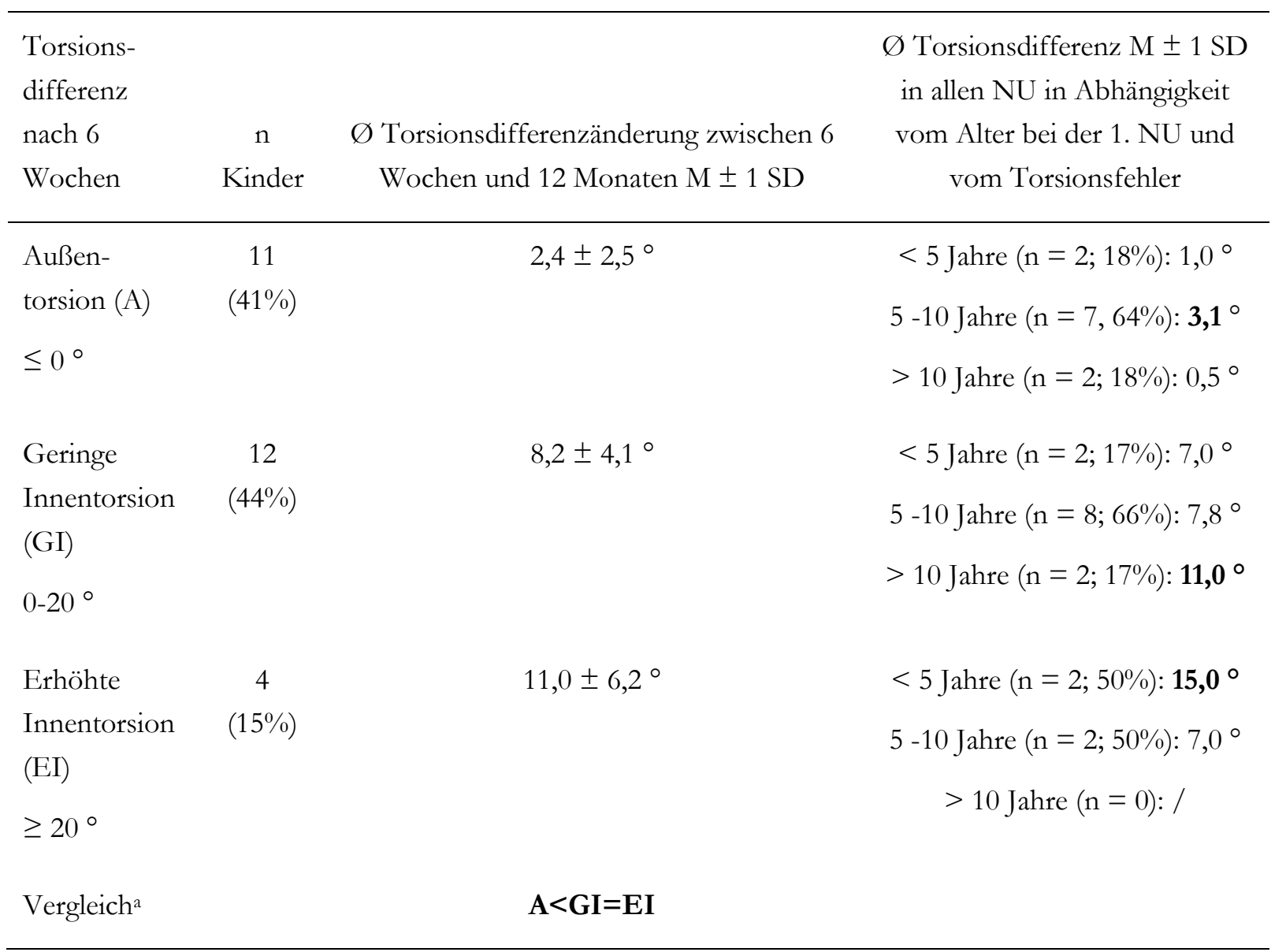

Anmerkung. $\mathrm{M}=$ Mittelwert. $\mathrm{SD}=$ Standardabweichung.

${ }^{a}$ Vergleich: Kruskal-Wallis Test mit Dunns Verteilung

Tabelle 34 zeigt die durchschnittliche Torsionsdifferenz in allen Nachuntersuchungszeiträumen an, aufgeteilt auf die Altersstruktur der Kinder bei der NU nach sechs Wochen. In allen Altersgruppen kam es zu einer Detorsion in den weiteren Nachuntersuchungen. Der größte Unterschied zeigte sich bei den Kindern, die jünger als fünf Jahre waren.

Es ergaben sich signifikante Unterschiede in der Gruppe der < 5-Jährigen zwischen sechs Wochen und zwölf Monate. In der Gruppe der 5- bis 10-Jährigen gab es signifikante Unterschiede zwischen sechs Wochen und vier Monate, sechs Wochen und sechs Monate sowie zwischen sechs Wochen und zwölf Monate. Bei den Kindern, die > zehn Jahre alt waren, war der Unterschied nicht signifikant. 
Tabelle 34: Torsionsdifferenz bei allen Nachuntersuchungszeitpunkten in Abhängigkeit vom Alter bei der NU nach sechs Wochen bis zwölf Monaten

\begin{tabular}{|c|c|c|c|c|c|}
\hline Altersgruppe & $\begin{array}{l}6 \text { Wochen } \\
M \pm 1 \mathrm{SD}\end{array}$ & $\begin{array}{l}4 \text { Monate } \\
\mathrm{M} \pm 1 \mathrm{SD}\end{array}$ & $\begin{array}{l}6 \text { Monate } \\
\mathrm{M} \pm 1 \mathrm{SD}\end{array}$ & $\begin{array}{l}12 \text { Monate } \\
\mathrm{M} \pm 1 \mathrm{SD}\end{array}$ & $\begin{array}{l}\text { Vergleich je } \\
\text { NU b }\end{array}$ \\
\hline $\begin{array}{l}\text { Kinder }<5 \text { Jahre } \\
(\mathrm{n}=6)\end{array}$ & $14,3 \pm 12,7^{\circ}$ & $8,7 \pm 10,3^{\circ}$ & $9,3 \pm 8,8^{\circ}$ & $6,7 \pm 7,3^{\circ}$ & $6 \mathrm{~W}>12 \mathrm{M}$ \\
\hline $\begin{array}{l}\text { Kinder } 5 \text {-10 Jahre } \\
(\mathrm{n}=17 \mathrm{a})\end{array}$ & $9,0 \pm 7,2^{\circ}$ & $6,2 \pm 6,7^{\circ}$ & $4,9 \pm 5,5^{\circ}$ & $3,6 \pm 5,4^{\circ}$ & $\begin{aligned} 6 \mathrm{~W} & >4 \mathrm{M}=6 \mathrm{M} \\
& =12 \mathrm{M}\end{aligned}$ \\
\hline $\begin{array}{l}\text { Kinder }>10 \text { Jahre } \\
(n=4)\end{array}$ & $6,5 \pm 6,4^{\circ}$ & $2,8 \pm 2,8^{\circ}$ & $1,8 \pm 1,8^{\circ}$ & $0,8 \pm 1,0^{\circ}$ & $\begin{aligned} 6 \mathrm{~W} & =4 \mathrm{M}=6 \mathrm{M} \\
& =12 \mathrm{M}\end{aligned}$ \\
\hline Vergleich je Alter ${ }^{c}$ & $\begin{array}{c}\text { Kein } \\
\text { Unterschied }\end{array}$ & $\begin{array}{c}\text { Kein } \\
\text { Unterschied }\end{array}$ & $\begin{array}{c}\text { Kein } \\
\text { Unterschied }\end{array}$ & $\begin{array}{c}\text { Kein } \\
\text { Unterschied }\end{array}$ & \\
\hline
\end{tabular}

Anmerkung. $\mathrm{M}=$ Mittelwert. $\mathrm{SD}=$ Standardabweichung.

${ }^{\mathrm{a}} \mathrm{n}=17$ : nach 6 Monaten $\mathrm{n}=16^{\mathrm{b}}$ Vergleich je NU: repeated measures $A N O V A$ mit Bonferroni Verteilung in der Gruppe der < 5-Jährigen und 5- bis 10-Jährigen. In der Gruppe der > 10Jährigen: Friedman-Test mit Dunns Verteilung. c Vergleich je Alter: Kruskal-Wallis Test mit Dunns Verteilung. $6 \mathrm{~W}=4 \mathrm{M}=6 \mathrm{M}=12 \mathrm{M}$ wenn $\mathrm{p}>0,05$ und Zeichen $<$ bzw. $>$ bedeuten $\mathrm{p}<0,05$

\subsubsection{Humerustorsionsdifferenz in Korrelation zum Cubitus varus / valgus}

Bei 21 Kindern bestand zum Zeitpunkt sechs Wochen nach Unfall noch keine wiederhergestellte Ellenbogenextension, sodass die Ellenbogenachse nicht beurteilt werden konnte. Nach vier Monaten hatten 13 von 17 Kindern in der Gruppe mit der Humerustorsionsdifferenz von $\leq 5^{\circ}$ eine freie Ellenbogenextension und in der Gruppe mit einer Humerutorsionsdifferenz von $>5^{\circ}$ acht von zehn Kindern. Es ließen sich beim Vergleich der Gruppen keinerlei signifikante Unterschiede finden (Tabelle 35). Einen Cubitus varus auf der frakturierten Seite gab es bei $\leq 5^{\circ}$ Humerustorsionsdifferenz nicht. Bei der Humerustorsionsdifferenz von $>5^{\circ}$ bei der NU nach zwölf Monaten gab es ein Kind mit einem Cubitus varus auf der frakturierten Seite. 
Tabelle 35: Humerustorsionsdifferenz bei der NU nach vier Monaten im Vergleich zur Ellenbogenachsendifferenz zwischen dem gesunden und frakturierten Arm in den folgenden Nachuntersuchungen

\begin{tabular}{lccc}
\hline $\begin{array}{c}\text { Humerustorsions- } \\
\text { differenz nach } 4 \\
\text { Monaten }\end{array}$ & 4 Monate & 12 Monate & \\
\hline$\leq 5^{\circ}\left(\varnothing 1,6^{\circ}\right)$ & $1,9 \pm 2,5^{\circ}$ & $\mathrm{M} \pm 1 \mathrm{SD}$ & $\mathrm{p}-$ Wert $^{\mathrm{a}}$ \\
$(\mathrm{n}=17)$ & $(\mathrm{n}=13)$ & $1,5 \pm 3,0^{\circ}$ & $\mathrm{p}>0,05$ \\
& $0,6 \pm 1,8^{\circ}$ & $(\mathrm{n}=17)$ & \\
$>5^{\circ}\left(\varnothing 14^{\circ}\right)$ & $(\mathrm{n}=8)$ & $2,5 \pm 4,9^{\circ}$ & $\mathrm{p}>0,05$ \\
$(\mathrm{n}=10)$ & $(\mathrm{n}=10)$ & \\
$\mathrm{p}-\mathrm{Wert}^{\mathrm{b}}$ & $\mathrm{p}>0,05$ & $\mathrm{p}>0,05$ & \\
\hline
\end{tabular}

Anmerkung. $\mathrm{M}=$ Mittelwert. $\mathrm{SD}=$ Standardabweichung. Die unterschiedlichen Untersuchungszahlen (n) kommen deshalb zustande, da bei den einzelnen Nachuntersuchungen die Achsen der Kinder noch nicht wieder hergestellt waren. ${ }^{a}$ p-Wert: Wilcoxon matched pairs Test. ${ }^{b}$ p- Wert: Mann-Whitney Test

\subsubsection{Humerustorsionsdifferenz und Funktion von Schulter/ Ellenbogen}

Es bestand kein Zusammenhang vom Ausmaß der Humerustorsionsdifferenz nach 6 Wochen und dem Grad der Schulteraußenrotationsdifferenz in den Nachuntersuchungen (Tabelle 36). Tendenziell (nicht signifikant) ging eine erhöhte sonographisch gemessene Humerustorsionsdifferenz von $>5^{\circ}$ mit einer erhöhten Schulteraußenrotationsdifferenz einher. Es fand sich nur ein signifikanter Unterschied innerhalb der NU nach 12 Monaten. Bei allen anderen Berechnungen gab es keine Signifikanzen.

Tabelle 36: Ergebnisse der Untersuchung der Humerustorsionsdifferenz im Verhältnis zur Schulteraußenrotationsdifferenz in den nachfolgenden Nachuntersuchungen

\begin{tabular}{lcclc}
\hline $\begin{array}{c}\text { Humerustorsions- } \\
\text { differenz nach 6 Wochen }\end{array}$ & $\mathrm{n}$ & $\begin{array}{c}\text { 6 Wochen } \\
\mathrm{M} \pm 1 \mathrm{SD}\end{array}$ & $\begin{array}{c}12 \text { Monate } \\
\mathrm{M} \pm 1 \mathrm{SD}\end{array}$ & $\mathrm{p}$ - Wert \\
\hline$\leq 5^{\circ}\left(\varnothing 1,4^{\circ}\right)$ & 9 & $3,9 \pm 6,0^{\circ}$ & $2,2 \pm 4,4^{\circ}$ & $\mathrm{p}>0,05$ \\
$>5^{\circ}\left(\varnothing 14^{\circ}\right)$ & 18 & $10,0 \pm 10,9^{\circ}$ & $7,8 \pm 8,1^{\circ}$ & $\mathrm{p}>0,05$ \\
$\mathrm{p}-$ Wert $^{\mathrm{b}}$ & & $\mathrm{p}>0,05$ & $\mathrm{p}<\mathbf{0 , 0 5}$ & \\
\hline
\end{tabular}


Anmerkung. $\mathrm{M}=$ Mittelwert. $\mathrm{SD}=$ Standardabweichung. ${ }^{\mathrm{a}} \mathrm{p}$-Wert: Wilcoxon matched pairs Test bei einer Humerustorsionsdifferenz von $\leq 5^{\circ}$ und Paired t-Test bei einer Humerustorsionsdifferenz von $>5^{\circ} \cdot{ }^{\mathrm{b}} \mathrm{p}$-Wert: Unpaired t-Test bei Nachuntersuchung nach 6 Wochen und Mann-Whitney Test bei Nachuntersuchung nach 12 Monaten

Bei der Humerustorsionsdifferenz nach sechs Wochen in Korrelation zu der Schulterinnenrotationsdifferenz in den Nachuntersuchungen ergab sich kein Zusammenhang (Tabelle 37). Aber auch hier zeigten sich bei der erhöhten sonographisch gemessenen Humerustorsionsdifferenz von $>5^{\circ}$ tendenziell (nicht signifikant) erhöhte Schulterinnenrotationsdifferenzwerte.

Tabelle 37: Ergebnisse der Untersuchung der Humerustorsionsdifferenz nach 6 Wochen im Verhältnis zur Schulterinnenrotationsdifferenz in den Nachuntersuchungen

\begin{tabular}{lcccc}
\hline $\begin{array}{c}\text { Humerustorsions- } \\
\text { differenz nach 6 Wochen }\end{array}$ & $\mathrm{n}$ & $\mathrm{M} \pm 1 \mathrm{SD}$ & $\mathrm{M} \pm 1 \mathrm{SD}$ & $\mathrm{p}^{-W_{\text {Wert }}{ }^{\mathrm{a}}}$ \\
\hline$\leq 5^{\circ}\left(\varnothing 1,4^{\circ}\right)$ & 9 & $5,6 \pm 7,3^{\circ}$ & $6,7 \pm 7,1^{\circ}$ & $\mathrm{p}>0,05$ \\
$>5^{\circ}\left(\varnothing 14^{\circ}\right)$ & $18^{\mathrm{a}}$ & $8,1 \pm 10,2^{\circ}$ & $7,8 \pm 8,1^{\circ}$ & $\mathrm{p}>0,05$ \\
$\mathrm{p}-$ Wert $^{\mathrm{b}}$ & & $\mathrm{p}>0,05$ & $\mathrm{p}>0,05$ & \\
\hline
\end{tabular}

Anmerkung. $\mathrm{M}=$ Mittelwert. $\mathrm{SD}=$ Standardabweichung.

${ }^{a}$ p-Wert: Paired t-Test. ${ }^{b}$ p- Wert: Unpaired t-Test

Bei der Ellenbogenextension bestand ein größeres Streckdefizit, wenn die Humerustorsionsdifferenz bei der NU nach 6 Wochen bei $>5^{\circ}$ lag (Tabelle 38). Die Extensionsdifferenz entwickelte sich in beiden Gruppen $\left(\leq 5^{\circ}\right.$ und $>5^{\circ}$ Humerustorsionsdifferenz) im Verlauf von einer erheblichen Differenz zu keiner bzw. fast keiner Differenz zwischen dem gesunden und frakturierten Oberarm. Innerhalb der Untersuchung nach sechs Wochen zeigte sich ebenfalls ein signifikanter Unterschied.

Zusammenfassend kann gesagt werden, dass Kinder, die eine Humerustorsionsdifferenz von $>5^{\circ}$ hatten, eine signifikant schlechtere Ellenbogenextension aufwiesen. 
Tabelle 38: Ergebnisse der Untersuchung der Humerustorsionsdifferenz nach 6 Wochen im Vergleich zur Ellenbogenextensionsdifferenz in den Nachuntersuchungen

\begin{tabular}{lcccc}
\hline $\begin{array}{c}\text { Humerustorsions- } \\
\text { differenz nach 6 Wochen }\end{array}$ & $\mathrm{n}$ & $\mathrm{M} \pm$ Wochen & 12 Monate & \\
\hline$\leq 5^{\circ}\left(\varnothing 1,4^{\circ}\right)$ & 9 & $32,8 \pm 14,2^{\circ}$ & $0,0 \pm 0,0^{\circ}$ & $/$ \\
$>5^{\circ}\left(\varnothing 14^{\circ}\right)$ & $18^{\mathrm{a}}$ & $43,6 \pm 22,0^{\circ}$ & $1,1 \pm 4,7^{\circ}$ & $\mathbf{p}<\mathbf{0 , 0 5}$ \\
$\mathrm{p}-$ Wert $^{\mathrm{b}}$ & & $\mathbf{p}<\mathbf{0 , 0 5}$ & $/$ & \\
\hline
\end{tabular}

Anmerkung. $\mathrm{M}=$ Mittelwert. $\mathrm{SD}=$ Standardabweichung. $/$ = statistische Berechnung wegen der Differenz von $0^{\circ}$ bei der NU nach 12 Monaten nicht möglich. ${ }^{a}$ p-Wert: Wilcoxon matched pairs Test. ${ }^{b}$ p-Wert: Mann-Whitney Test

Ein vorhandenes Ellenbogenflexionsdefizit - gemessen als Differenz zur gesunden Gegenseite - besserte sich im Verlauf der Untersuchungen. Die größte Besserung (auch signifikant unterschiedlich) fand bei einer Humerustorsionsdifferenz von $>5^{\circ}$ statt (Tabelle 39).

Tabelle 39: Ergebnisse der Untersuchung der Humerustorsionsdifferenz nach 6 Wochen im Vergleich zur Ellenbogenflexionsdifferenz in den Nachuntersuchungen

\begin{tabular}{lcccc}
\hline $\begin{array}{c}\text { Humerustorsions- } \\
\text { differenz nach 6 Wochen }\end{array}$ & $\mathrm{n}$ & $\mathrm{M} \pm 1 \mathrm{SD}$ & $\mathrm{M} \pm 1 \mathrm{SD}$ & p-Wert $^{\mathrm{a}}$ \\
\hline$\leq 5^{\circ}\left(\varnothing 1,4^{\circ}\right)$ & 9 & $10,6 \pm 7,3^{\circ}$ & $0,0 \pm 0,0^{\circ}$ & $/$ \\
$>5^{\circ}\left(\varnothing 14^{\circ}\right)$ & 18 & $14,4 \pm 9,7^{\circ}$ & $1,4 \pm 4,1^{\circ}$ & $\mathrm{p}<\mathbf{0 , 0 5}$ \\
$\mathrm{p}-$ Wert $^{\mathrm{b}}$ & & $\mathrm{p}>0,05$ & $/$ & \\
\hline
\end{tabular}

Anmerkung. $\mathrm{M}=$ Mittelwert. $\mathrm{SD}=$ Standardabweichung. $/$ = statistische Berechnung wegen der Differenz von $0{ }^{\circ}$ bei der NU nach 12 Monaten nicht möglich. ${ }^{a}$ p-Wert: Wilcoxon matched pairs Test. ${ }^{b}$ p- Wert: Unpaired t-Test

\subsubsection{Humerustorsionsveränderung und Analyse der Röntgenbilder}

Ein seitliches Röntgenbild wurde drei Wochen nach der Operation zur Feststellung der Frakturkonsolidation angefertigt. Hierauf wurde ein potentieller Rotationssporn analysiert und mit ,ja“ oder „nein“ in den Ergebnissen notiert (Kapitel 2.2 und 2.5). Es fanden sich radiologisch Rotationssporne sowohl bei der geringeren als auch bei der größeren Humerustorsionsdifferenz, gemessen bei der NU nach sechs Wochen. Eine Korrelation 
des radiologisch identifizierten Humerustorsionsfehlers - gemessen als Rotationssporn bzw. als Achsenfehlstellung - mit der sonographischen Humerustorsionsdifferenz erfolgte in Tabelle 40.

Tabelle 40: Ergebnisse der Untersuchung der Humerustorsionsdifferenz nach 6 Wochen im Vergleich zu den Röntgenaufnahmen mit radiologischen Auffälligkeiten wie z. B. Rotationssporn im Seitbild, Achsfehlstellungen)

\begin{tabular}{cc}
\hline $\begin{array}{c}\text { Humerustorsionsdifferenz } \\
\text { nach } 6 \text { Wochen }\end{array}$ & $\begin{array}{c}\text { Röntgenbefund nach } 3 \text { Wochen und } \\
\text { Anzahl der Kinder }\end{array}$ \\
\hline $5^{\circ}\left(\varnothing 1,4^{\circ}\right)$ & Varus: $1(11 \%)$ \\
$\mathrm{n}=9$ & Valgus $1(11 \%)$ \\
& Kein Sporn, keine Achsfehlstellu:ng, kein \\
Bild: $7(78 \%)$ \\
Sporn: $5(27 \%)$ \\
Varus: $1(6 \%)$ \\
Valgus: $1(6 \%)$ \\
Abkippen nach dorsal: $1(6 \%)$ \\
Kein Sporn, keine Achsfehlstellung, kein \\
Bild: $10(55 \%)$
\end{tabular}

Bei den Kindern ohne Humerustorsionsdifferenz in der sechs Wochen Messung $(n=9)$ fanden sich in 78\% der Fälle radiologisch kein Rotationssporn oder Achsabweichungen. Zwei Röntgenbilder (22\%) wiesen Achsfehlstellungen auf.

Bei sonographisch gemessener Humerustorsionsdifferenz $\left(>5^{\circ}\right)$ fanden sich im Röntgenbild nach drei Wochen in 44\% der Fälle Auffälligkeiten. Diese setzten sich zusammen aus fünf Rotationsspornen, einem Cubitus Varus, einem Cubitus Valgus und einer Abkippung nach dorsal.

\subsubsection{Humerustorsionsdifferenz im Verhältnis zur Händigkeit}

Vom gesamten Kollektiv ( $=27$ ) waren 23 Kinder rechtshändig, drei Kinder beidhändig und ein Kind linkshändig. Von diesen Kindern erlitten zwölf Kinder (44\%) eine Fraktur an der händigen Extremität, wobei bei beidhändigen Patienten $(n=3)$ die Fraktur zur dominanten Seite eingestuft wurde. Wegen der potentiellen schnelleren Änderung der posttraumatischen Humerustorsion durch vermehrten Gebrauch der Extremität (= Händigkeit), wurden Frakturen auf der dominanten, händigen Seite, mit Frakturen auf der nicht dominanten Seite im Verlauf verglichen (Tabelle 41). Hier fand sich kein signifikanter Unterschied. 
Tabelle 41: Humerustorsionsdifferenz $(M \pm 1 S D)$ in Abhängigkeit der Händigkeit

$\varnothing$ Humerustorsionsdifferenz

\begin{tabular}{|c|c|c|c|}
\hline $\begin{array}{l}\text { Nachuntersuchungs- } \\
\text { zeitpunkte }\end{array}$ & $\begin{array}{l}\text { Fraktur auf Seite des } \\
\text { dominanten Armes } \\
\qquad(\mathrm{n}=12)\end{array}$ & $\begin{array}{c}\text { Fraktur auf Seite des } \\
\text { nicht dominanten } \\
\text { Armes }\left(\mathrm{n}=15^{\mathrm{a}}\right)\end{array}$ & $\mathrm{p}-$ Wert $^{\mathrm{b}}$ \\
\hline 6 Wochen $(6 \mathrm{~W})$ & $9,9 \pm 9,9^{\circ}$ & $9,7 \pm 7,9^{\circ}$ & $p>0,05$ \\
\hline 4 Monate (4M) & $5,9 \pm 8,2^{\circ}$ & $6,5 \pm 6,6^{\circ}$ & $\mathrm{p}>0,05$ \\
\hline 6 Monate $(6 \mathrm{M})$ & $6,6 \pm 7,2^{\circ}$ & $4,4 \pm 5,7^{\circ}$ & $p>0,05$ \\
\hline 12 Monate $(12 \mathrm{M})$ & $3,5 \pm 5,4^{\circ}$ & $4,2 \pm 6,0^{\circ}$ & $\mathrm{p}>0,05$ \\
\hline Vergleichc & $\begin{array}{c}6 \mathrm{~W}>4 \mathrm{M}=12 \mathrm{M} \\
6 \mathrm{~W}=6 \mathrm{M} \\
4 \mathrm{M}=6 \mathrm{M}=12 \mathrm{M}\end{array}$ & $6 \mathrm{~W}>4 \mathrm{M}=6 \mathrm{M}=12 \mathrm{M}$ & \\
\hline
\end{tabular}

Anmerkung. $\mathrm{M}=$ Mittelwert. $\mathrm{SD}=$ Standardabweichung. ${ }^{\mathrm{a}} \mathrm{n}=15$ : nach 6 Monaten $\mathrm{N}=14$.

b p-Wert: Unpaired t-Test". " Vergleich: repeated measures ANOVA mit Bonferroni Verteilung. $6 \mathrm{~W}=4 \mathrm{M}=6 \mathrm{M}=12 \mathrm{M}$ wenn $\mathrm{p}>0,05$ und Zeichen $<$ bzw. $>$ bedeuten $\mathrm{p}<0,05$

Es zeigte sich bei der Humerustorsionsdifferenzänderung kein Unterschied in Bezug auf die Händigkeit der Kinder und damit den dominanten Armgebrauch. 


\subsection{Normalkollektiv}

\subsubsection{Daten des Normalkollektivs}

Das untersuchte Normalkollektiv bestand aus insgesamt 250 gesunden oberen Extremitäten, davon 150 Kinderextremitäten und 100 Erwachsenenextremitäten.

Aus diesem Datensatz wurden bei 51 Kindern und 50 Erwachsenen die Differenzen der Humerustorsion intraindividuell ermittelt. In der Promotionsarbeit von Christoph Katthagen 2008 und in der Publikation von Katthagen et al. 2009 sind diese Daten ebenfalls verwendet und veröffentlicht worden.

Die durchschnittliche Humerustorsion auf der rechten Seite betrug hier 17,5 $\pm 11,5^{\circ}$ und auf der linken Seite 19,7 $\pm 12,9^{\circ}$. Die Differenz zwischen der rechten und linken Seite des Armes betrug dabei 5,0 $\pm 4,4^{\circ}$. Dieser Unterschied war nicht signifikant.

Die durchschnittliche Humerustorsion im Normalkollektiv Erwachsene mit N=50 betrug auf der rechten Seite $22,2 \pm 10,0^{\circ}$ und auf der linken Seite $25,7 \pm 10,6^{\circ}$. Mithilfe des Paired t-Test für abhängige Messungen konnte ein signifikanter Unterschied zwischen dem rechten und linken Arm errechnet werden (Tabelle 42). Die Altersspanne der Erwachsenen reichte von 21,9 bis 63,5 Jahre.

Für das Normalkollektiv Kinder mit N=51, wo beide oberen Extremitäten gleichzeitig gemessen wurden, ergab sich auf der rechten Seite eine durchschnittliche Humerustorsion von $12,9 \pm 11,1^{\circ}$ und auf der linken Seite von 13,9 $\pm 12,3^{\circ}$. Zur Signifikanzprüfung wurde auch hier ein Paired t-Test für abhängige Messungen und normal verteilte Werte gerechnet. Es ergab sich hier kein signifikanter Unterschied zwischen dem rechten und linken Arm des Kinder-Normalkollektivs mit N=51 (Tabelle 42). 
Tabelle 42: Mittelwert ( \pm 1 Standardabweichung) der Humerustorsion in den Normalkollektiven

\begin{tabular}{lcccc}
\hline Kollektiv & $\mathrm{n}$ & Arm rechts & Arm links & p-Wert \\
\hline Normalkollektiv Erwachsene & 50 & $22,2 \pm 10,0^{\circ}$ & $25,7 \pm 10,6^{\circ}$ & $\mathbf{p}<\mathbf{0 , 0 5}$ \\
$\begin{array}{l}\text { Kinder-Normalkollektiv, } \\
\text { beide Extremitäten }\end{array}$ & 51 & $12,9 \pm 11,1^{\circ}$ & $13,9 \pm 12,3^{\circ}$ & $\mathrm{p}>0,05$ \\
$\begin{array}{l}\text { Kinder- und Erwachsenenkollektiv } \\
\text { mit beiden oberen Extremitäten }\end{array}$ & 101 & $17,5 \pm 11,5^{\circ}$ & $19,7 \pm 12,9^{\circ}$ & $\mathbf{p}<\mathbf{0 , 0 5}$ \\
\hline
\end{tabular}

Das durchschnittliche Alter der 51 Kinder betrug zum Zeitpunkt der Untersuchung 9,9 \pm 3,5 Jahre (von 1,7 bis 17,2 Jahren; Abbildung 23) und bei den 50 Erwachsenen 29,4 \pm 9,7 Jahre (von 21,9 bis 63,5 Jahren; vergleiche Dissertation Katthagen 2008).

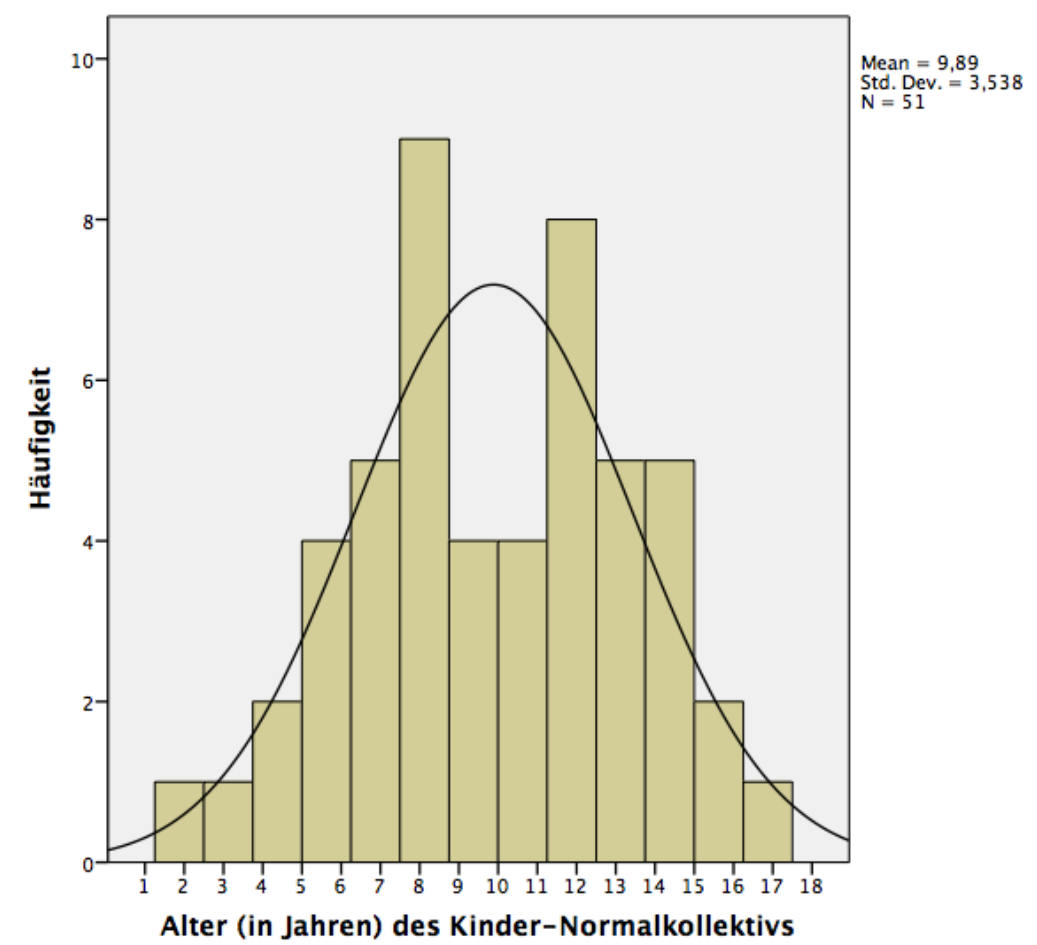

Abbildung 23: Altersverteilung des Kinder-Normalkollektivs

In der Tabelle 43 wurde das durchschnittliche Alter der Kinder im retrospektiven, prospektiven und im Normalkollektiv dargestellt. 
Tabelle 43: Durchschnittliches Alter der Kinderkollektive (retrospektives-, prospektivesund Normalkollektiv)

\begin{tabular}{lcc}
\hline \multicolumn{1}{c}{ Kollektiv } & N & Alter \\
\hline Retrospektives Kollektiv & 22 & $6,8 \pm 2,6$ \\
Prospektives Kollektiv & 27 & $6,5 \pm 2,5$ \\
Normalkollektiv & 51 & $9,9 \pm 3,5$ \\
\hline
\end{tabular}

Anmerkung. $\mathrm{M}=$ Mittelwert. $\mathrm{SD}=$ Standardabweichung

Zur Analyse, ob es einen signifikanten Unterschied im Alter bei den Gruppen zueinander gab, wurde hier eine one-way analysis of variance mit Bonferroni Verteilung gerechnet, da es keine Messwiederholungen gab.

Es gab einen signifikanten Unterschied zwischen dem Normalkollektiv zum retrospektiven Kollektiv sowie zwischen dem Normalkollektiv und dem prospektiven Kollektiv.

\subsubsection{Entwicklung der Humerustorsion im pädiatrischen Normalkollektiv und im prospektiven Kollektiv}

Wie schon im Kapitel 3.3.1 Daten des Normalkollektivs erwähnt, gab es keinen signifikanten Unterschied zwischen dem rechten und linken Arm im Normalkollektiv Kinder mit $\mathrm{N}=51$. Es zeigte sich jedoch eine Zunahme der Humerustorsion mit steigendem Alter sowohl beim rechten als auch dem linken Arm (Abbildung 24).

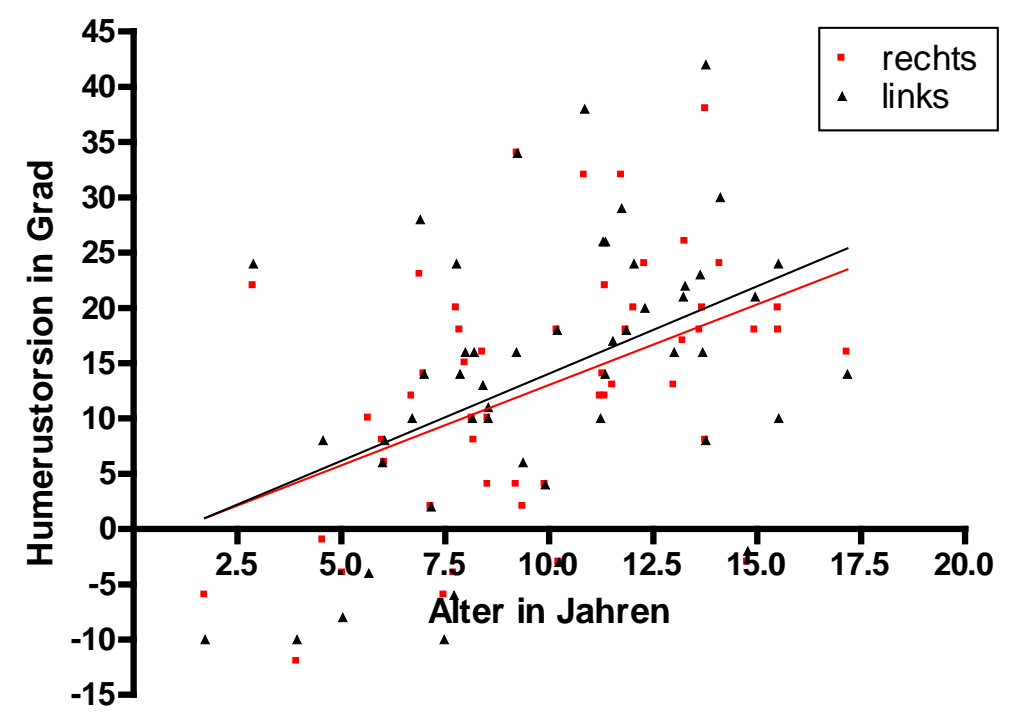

Abbildung 24: Verlauf der sonographisch gemessenen Humerustorsion mit zunehmendem Alter zwischen rechtem und linkem Arm im Kinder-Normalkollektiv ( $\mathrm{n}=51$ ) 
Bei der Analyse der Humerustorsionsdifferenz zwischen frakturierter und gesunder Seite von Kindern des prospektiven Kollektivs mit vorhandener Differenz $>5^{\circ}$ im Altersverlauf ( $\mathrm{n}=18)$ zeigte sich eine Annäherung bei der Regressionsgeraden (Abbildung 25).

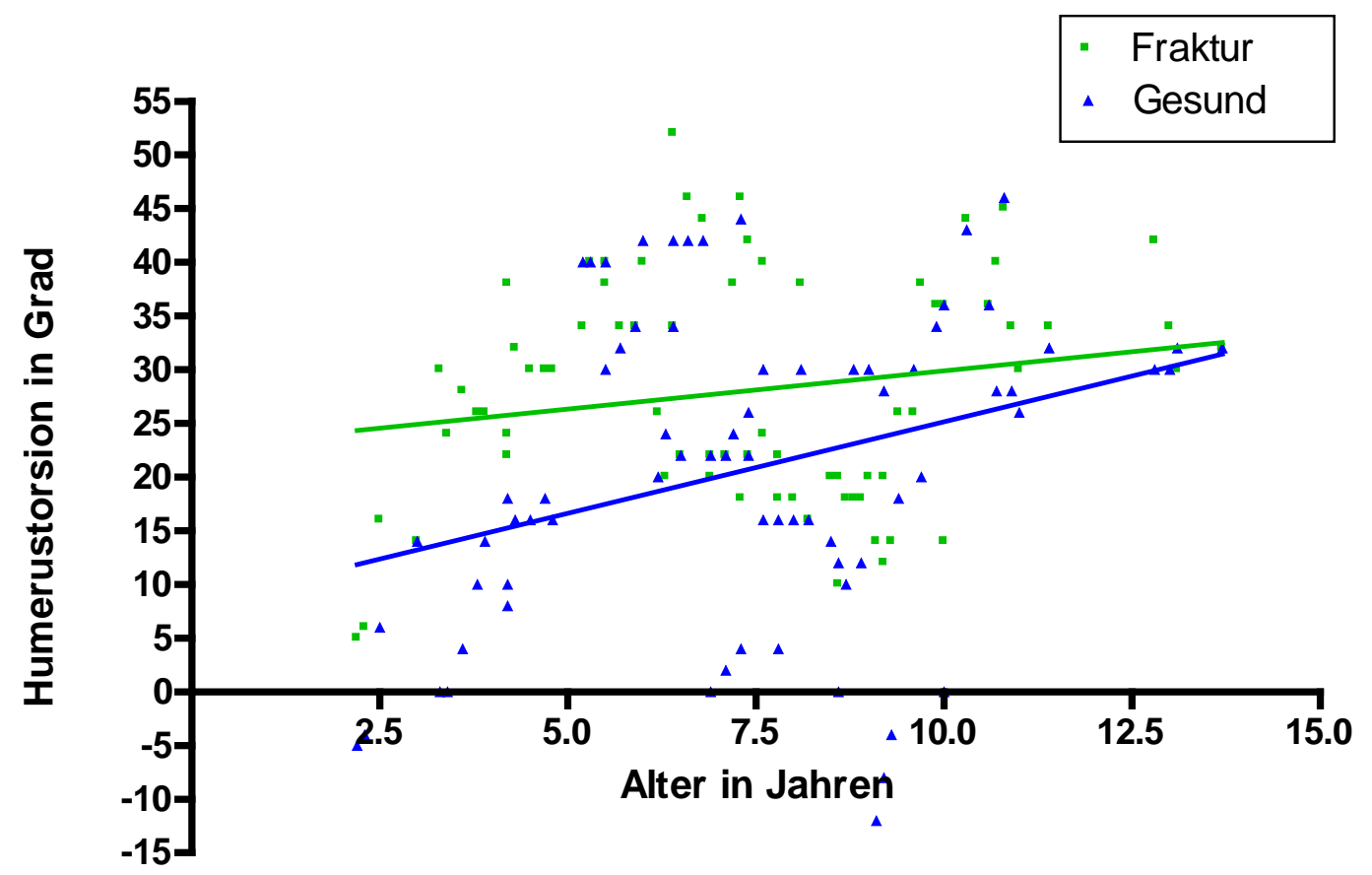

Abbildung 25: Humerustorsion der frakturierten und gesunden oberen Extremität im prospektiven Kollektiv von Kindern mit vorhandener Humerustorsionsdifferenz $(n=18)$ im Altersverlauf

Die gesamte Entwicklung der Humerustorsion des Kinder-Normalkollektivs aufgeteilt auf die drei Altersgruppen ist in der Tabelle 44 dargestellt. Mit steigendem Alter nahm auch die Humerustorsion zu.

Tabelle 44: Gesamte sonographisch gemessene Humerustorsion (rechts und links) im Kinder-Normalkollektiv in unterschiedlichen Altersgruppen

Altersgruppe $<5$ Jahre $(\mathrm{n}=4) \quad 5$-10 Jahre $(\mathrm{n}=22) \quad \begin{gathered}10-16 \text { Jahre } \\ (\mathrm{n}=25)\end{gathered}$

$\mathrm{M} \pm 1 \mathrm{SD} \quad 1,9 \pm 14,6^{\circ} \quad 9,5 \pm 10,2^{\circ} \quad 18,6 \pm 9,8^{\circ}$

Anmerkung. $\mathrm{M}=$ Mittelwert, $\mathrm{SD}=$ Standardabweichung

In der nachfolgenden Tabelle 45 wurde das Normalkollektiv der Kinder $(\mathrm{N}=51)$ in drei verschiedene Altersgruppen ( $<5$ Jahre; 5 -10 Jahre; $>10$-16 Jahre) aufgeteilt und die jeweils sonographisch gemessenen Humerustorsion der rechten und linken Seite der oberen Extremität notiert. In den jeweiligen Gruppen konnte zwischen dem rechten und linken Arm kein signifikanter Unterschied festgestellt werden. Allerdings kam es aber zu 
signifikanten Unterschieden auf der rechten Armseite zwischen der Gruppe 1 und 3 sowie auf der linken Armseite zwischen der Gruppe 1 und 2 und Gruppe 1 und 3.

Tabelle 45: Durchschnittliche Humerustorsion des Normalkollektivs aufgeteilt in drei Altersgruppen

\begin{tabular}{|c|c|c|c|c|}
\hline \multirow{4}{*}{$\begin{array}{c}\text { Seite des } \\
\text { Armes }\end{array}$} & \multicolumn{3}{|c|}{ Altersgruppe } & \\
\hline & Gruppe 1 & Gruppe 2 & Gruppe 3 & \\
\hline & $\begin{array}{c}(<5 \text { Jahre, } \mathrm{n}=4) \\
\mathrm{M} \pm 1 \mathrm{SD}\end{array}$ & $\begin{array}{c}(5-10 \text { Jahre, } \\
n=22)\end{array}$ & $\begin{array}{c}(>10-16 \text { Jahre, } \\
n=25)\end{array}$ & \\
\hline & & $\mathrm{M} \pm 1 \mathrm{SD}$ & $\mathrm{M} \pm 1 \mathrm{SD}$ & Vergleicha \\
\hline Rechts & $0,8 \pm 14,9^{\circ}$ & $9,4 \pm 9,5^{\circ}$ & $17,9 \pm 9,3^{\circ}$ & Gruppe $1<3$ \\
\hline Links & $3,0 \pm 16,4^{\circ}$ & $9,7 \pm 11,0^{\circ}$ & $19,3 \pm 10,4^{\circ}$ & Gruppe $1<2=3$ \\
\hline $\mathrm{p}$-Wert ${ }^{\mathrm{b}}$ & $\mathrm{p}>0,05$ & $\mathrm{p}>0,05$ & $\mathrm{p}>0,05$ & \\
\hline
\end{tabular}

Anmerkung. $\mathrm{M}=$ Mittelwert. $\mathrm{SD}=$ Standardabweichung.

${ }^{a}$ Vergleich: repeated measures ANOVA mit Bonferroni Verteilung. ${ }^{\mathrm{b}} \mathrm{p}-$ Wert: Paired t-Test. $2=3$ wenn $\mathrm{p}>0,05$ und $<$ bzw. $>$, wenn $\mathrm{p}<0,05$

\subsection{Inter-Observer-Untersuchung und Messfehler}

Zur Bestimmung des Inter-Observer-Messfehlers wurden von Untersucher A (AnnaKathrin Hell) und Untersucher B (Claudia Gadomski) bei 61 Probanden der rechte und der linke Arm gemessen (2.6. Validierung der Messmethode). Die Ergebnisse wurden in Tabelle 46 aufgelistet.

Tabelle 46: Mittelwerte ( \pm 1 Standardabweichung) der sonographisch gemessenen Humerustorsionen der jeweiligen Untersucher bei $\mathrm{N}=61$

\begin{tabular}{ccc}
\hline Untersucher & $\begin{array}{c}\text { Arm rechts } \\
\mathrm{M} \pm 1 \mathrm{SD}\end{array}$ & $\begin{array}{c}\text { Arm links } \\
\mathrm{M} \pm 1 \mathrm{SD}\end{array}$ \\
\hline Untersucher A & $21,4 \pm 9,8^{\circ}$ & $24,8 \pm 10,8^{\circ}$ \\
Untersucher B & $20,6 \pm 10,0^{\circ}$ & $24,5 \pm 10,8^{\circ}$ \\
Differenz & $2,0 \pm 1,4^{\circ}$ & $2,3 \pm 1,5^{\circ}$ \\
\hline
\end{tabular}

Anmerkung. $\mathrm{M}=$ Mittelwert. $\mathrm{SD}=$ Standardabweichung 
Die Analyse der Intraklassen-Korrelation ergab eine sehr hohe Übereinstimmung zwischen Untersucher A und Untersucher B sowohl für den rechten als auch für den linken Arm (für beide Arme betrug die Intraklassen-Korrelation $=0,97, \mathrm{p}<0,05)$. Der Messfehler dieser sonographischen Methode ergab für die rechte Seite 3,4 ${ }^{\circ}$ (Mittelwert \pm 1 Standardabweichung) und für die linke Seite $3,8^{\circ}$. Damit konnte ein mittlerer Messfehler von insgesamt $3,6^{\circ}$ berechnet werden (Katthagen et al. 2009).

\subsection{Gesamtzusammenfassung der Ergebnisse}

In der vorliegenden Arbeit wurden drei verschiedene Kollektive untersucht und ausgewertet:

Retrospektives Kollektiv ( $\mathbf{N = 2 2 )}$ mit dislozierten suprakondylären Humerusfrakturen, welche alle mit einem radialen Fixateur externe operativ versorgt worden waren. Alle Kinder $(\mathrm{N}=22)$ wurden $\mathrm{zu}$ unterschiedlichen Zeitpunkten (von vier Monaten bis dreieinhalb Jahren nach Fraktur) dreimal innerhalb von zwei Jahren mit jeweils zwölf Monaten Abstand anamnestisch, klinisch, photographisch und sonographisch nachuntersucht. Zur individuellen Analyse wurden folgende Untergruppen gebildet:

- Gruppe retro 1 ( $\mathrm{n}=10)$ : Zeit zwischen Unfall und 1. NU (Nachuntersuchung) unter einem Jahr

- Gruppe retro $2(\mathrm{n}=12):$ Zeit zwischen Unfall und 1. NU mehr als ein Jahr

Prospektives Kollektiv ( $\mathbf{N}=27)$ mit dislozierten suprakondylären Humerusfrakturen, welche alle mit gekreuzten K-Drähten operativ versorgt worden waren. Dieses prospektive Kollektiv wurde klinisch, photographisch und sonographisch jeweils nach sechs Wochen, vier Monaten, sechs Monaten und zwölf Monaten nach Fraktur nachuntersucht. Eine radiologische Kontrolle des Ellenbogens in zwei Ebenen erfolgte standardisiert nach drei Wochen.

Normalkollektiv ( $\mathrm{N}=50$ Erwachsene und $\mathrm{N}=99$ Kinder) mit intra- und interindividuellen Humerustorsionsmessungen zur Validierung der Methode und zur Erfassung der physiologischen Humerustorsionsentwicklung in Abhängigkeit vom Alter (Katthagen et al. 2009). Von den 99 Kindern wurden bei 51 Kindern beide oberen Extremitäten untersucht und bei 48 Kindern nur die gesunde Seite bei Fraktur am Gegenarm.

\section{Zusammenfassung der Ergebnisse des retrospektiven Kollektivs ( $\mathbf{N}=22)$}

In der Gruppe retro 1 (1. NU $<1$ Jahr nach Unfall) hatten bei der 1 . NU alle Kinder $(\mathrm{N}=10 ; 100 \%)$ eine Humerustorsionsdifferenz von $>5^{\circ}$. In der Gruppe retro $2(1 . \mathrm{NU}>$ 1 Jahr nach Unfall) war dies bei $75 \%$ der Kinder ( $n=9$ bei NU1) der Fall. Die Gruppe retro 1 zeigte eine größere Humerustorsionsdifferenzverminderung von NU1 zu NU3 (allerdings nicht signifikant) im Gegensatz zur Gruppe retro 2. 
Auf den Röntgenbildern in der Gruppe retro 1 fanden sich bei vorhandener Humerustorsionsdifferenz in 50\% ( $\mathrm{n}=5)$ der Fälle radiologische Auffälligkeiten. In der Gruppe retro 2 ohne Humerustorsionsdifferenz $\left(<5^{\circ}\right)$ gab es bei allen Kindern $(100 \% ; n=3)$ radiologische Auffälligkeiten und in der Gruppe retro 2 mit einer Humerustorsionsdifferenz von $>5^{\circ}$ bei $44 \%(n=4)$ der Kinder.

Für dieses Kollektiv bestand kein Zusammenhang zwischen der sonographisch gemessenen Humerustorsionsdifferenz und der Schulter- und Ellenbogenfunktion, der Ellenbogenachse bzw. einem Remodelling abhängig von der Richtung der Humerustorsionsdifferenz (innen/ außen) oder dem Alter.

\section{Zusammenfassung der Ergebnisse des prospektiven Kollektivs (N=27)}

Bei der ersten sonographischen Humerustorsionsanalyse sechs Wochen nach Unfall fanden sich bei 33\% ( $\mathrm{n}=9)$ der Kinder keine Humerustorsionsdifferenzen. 67\% ( $\mathrm{n}=18)$ wiesen eine Humerustorsionsdifferenz von $>5^{\circ}$ mit $\varnothing 14,0 \pm 7,6^{\circ}$ auf.

Auf den Röntgenbildern drei Wochen nach Fraktur fanden sich in der Gruppe ohne Humerustorsionsdifferenz ( $n=9)$ keine Rotationssporne und Normalbefunde in $78 \%$ $(\mathrm{n}=7)$. Zwei Röntgenbilder zeigten Achsabweichungen. In der Gruppe mit Humerustorsionsdifferenz fanden sich radiologische Auffälligkeiten in $45 \%(n=8)$ der Fälle.

Die größte signifikante Humerustorsionsdifferenzänderung erfolgte, wenn die Humerustorsion bei $\geq 20^{\circ}$ lag. Bei Kindern $<10$ Jahre $(\mathrm{n}=23)$ kam es zu signifikanten Humerusdetorsionen im Verlauf. Die größte signifikante Detorsion erfolgte bei Kindern $<5$ Jahre $(\mathrm{n}=6)$.

Keine signifikante Veränderung wurde bei der Analyse der Entwicklung der Humerustorsionsdifferenz zur Ellenbogenfunktion, Schulterfunktion und Ellenbogenachse gefunden.

\section{Zusammenfassung der Ergebnisse des Normalkollektivs, der Inter-Observer- Untersuchung und des Messfehlers}

Im Kinder-Normalkollektiv ( $\mathrm{N}=51)$ konnte gezeigt werden, dass die sonographisch gemessene Humerustorsion mit zunehmendem Alter anstieg (Abbildung 24), also eine vermehrte Innentorsion aufwies.

Zwischen Untersucher A und B gab es keinen signifikanten Unterschied. Der mittlere Messfehler betrug insgesamt $3,6^{\circ}$ (Katthagen et al. 2009). 


\section{Diskussion}

\subsection{Problematik der suprakondylären Humerusfraktur im Kindesalter}

Suprakondyläre Humerusfrakturen im Wachstumsalter können vielfältige Komplikationen verursachen: Gefäß- und Nervenläsionen, Funktionseinschränkungen der Ellenbogenextension und -Flexion sowie Veränderungen der Ellenbogenachse (Cubitus varus und valgus), Fehlstellungen in der Sagittalebene (Ante- und Rekurvation), Seit-zu-SeitVerschiebungen in der Horizontalebene und Torsionsfehler mit komplexen dreidimensionalen Deformitäten (Smith 1960; Graham 1967; Dowd und Hopcroft 1979; Labelle et al. 1982; France und Strong 1992; Chess et al. 1994; Mitsunari et al. 1995; Wilkins 1996; von Laer und Hasler 2000; Wessel et al. 2003). Während die Ellenbogenfunktion durch Remodelling der Knochenstrukturen in der Sagittalebene zumeist in den ersten sechs bis neun Monaten nach Unfall wiederhergestellt wird (von Laer 1979), ist der kosmetisch störende Cubitus varus ein persistierendes Problem. Zumeist ist die Achsfehlstellung auch mit einem Torsionsfehler vergesellschaftet. Aus klinischen Beobachtungen ist bekannt, dass Achs- und Torsionsdeformitäten nach suprakondylären Humerusfrakturen vermutlich wenig Remodellierungspotential aufweisen (von Laer 1979; Weinberg und Tscherne 2006; von Laer et al. 2013; Fernandez et al. 2016). Dies ist umso erstaunlicher, als beispielweise bei Torsionsfehlern nach Femurfrakturen im Kindesalter eine Korrektur im Verlauf stattfindet (Morscher 1961; von Laer 1977; Oberhammer 1980; Siebert und Pannike 1984; Buchholz et al. 2002; Spyra 2011).

In der vorliegenden Arbeit wird erstmals der posttraumatische Torsionsfehler nach suprakondylärer Humerusfraktur im Kindesalter erfasst und im zeitlichen Verlauf beobachtet.

\subsection{Konsequenz des posttraumatischen Torsionsfehlers}

Der Einfluss des Torsionsfehlers auf die Entstehung des Cubitus varus ist umstritten. Während Hindman et al. (1988) einen Cubitus varus bei einem Torsionsfehler über $10^{\circ}$ beschrieben, konnten andere Autoren (Smith 1960; Dowd und Hopcroft 1979; Mahaisavariya und Laupattarakasem 1993; von Laer und Hasler 2000) Cubitus-varusFehlstellungen ohne Torsionsfehler nachweisen. Aus Experimenten ist bekannt, dass die suprakondyläre Humerusfraktur mit zunehmender Torsionsfehlstellung instabiler wird bei meist zentralem Drehpunkt zwischen den Frakturfragmenten, also zwischen radialem und ulnarem Kondylenmassiv (Abbildung 8, von Laer et al. 2013). Ein zentraler Drehpunkt prädisponiert zum medialen (ulnaren) Kollaps der Fraktur, was wiederum eine 
Varusdeformität zur Folge hat (Smith 1960; Labelle et al. 1982; Mahaisavariya und Laupattarakasem 1993; Chess et al. 1994; Wilkins 1996).

Zur Behandlung der dislozierten, instabilen suprakondylären Humerusfraktur sind vielfältige Methoden beschrieben (von Laer 1997; Weise et al. 1997; Ababneh et al. 1998; de las Heras et al. 2005; Schäffer et al. 2007; von Laer et al. 2013). Im europäischen Raum werden die gekreuzte K-Draht-Osteosynthese, der ESIN und der radiale Fixateur externe (von Laer 1997; Slongo et al. 2008) häufig verwendet. Der von Lutz von Laer beschriebene radiale Fixateur externe als Osteosynthese bei der instabilen suprakondylären Humerusfraktur komprimiert beide Frakturfragmente auf dem radialen Kondylenmassiv (von Laer et al. 2013). Diese Methode basiert auf einer Studie, bei der gezeigt werden konnte, dass bei Kompression der Fragmente im Bereich des radialen Kondylus bei zunehmender Drehung die Kontaktflächen mit etwa 70\% konstant bleiben (von Laer 1997; von Laer et al. 2013, Abbildung 8). Hierdurch wird die Fraktur stabilisiert und ein mediales Abkippen verhindert (von Laer et al. 2013), gegebenenfalls auch unter Inkaufnahme eines Torsionsfehlers (Abbildung 10). Der Vorteil dieses Verfahren wird darin gesehen, dass kein ulnarer Draht eingebracht werden muss, wodurch das Risiko einer Schädigung des Nervus ulnaris reduziert wird. Diese Frakturversorgung wird als bewegungsstabil beschrieben (von Laer 1997; von Laer et al. 2013).

Der vorangegangene Aspekt der Frakturversorgung ist für die vorliegende Arbeit relevant, da das retrospektive Kollektiv $(\mathrm{N}=22)$ ausschließlich mit dem radialen Fixateur externe (von Laer 1997) und das prospektive Kollektiv ( $=27$ ) allein mit der Methode der gekreuzten K-Drähte (Scola et al. 2002) versorgt wurde. Durch die oben diskutierte Torsion der Fragmente gegeneinander bei radialer Kompression zeigte daher im retrospektiven Kollektiv eine größere Humerustorsionsdifferenz als Zeichen eines persistierenden posttraumatischen Torsionsfehlers gegenüber der Daten des prospektiven Kollektivs (Ø $18,5^{\circ}$ im retrospektiven Kollektiv versus $14^{\circ}$ im prospektiven Kollektiv). Zudem fand sich in der retrospektiven Gruppe (Gruppe retro 1) bei 100\% der Kinder eine Humerustorsionsdifferenz, welche in der prospektiven Gruppe nur in 67\% bei der ersten NU vorhanden war. Somit konnte für die Methode der gekreuzten K-Draht-Osteosynthese eine geringere Torsionsdifferenz nachgewiesen werden. Die Fixierungsart der gekreuzten K-Draht-Osteosynthese ist aktuell auch das gebräuchlichste Verfahren zur Versorgung dislozierter suprakondylärer Humerusfrakturen (Weise et al. 1997; Ababneh et al. 1998; Schofer et al. 2003; de las Heras et al. 2005; Schäffer et al. 2007). Allerdings konnte in der vorliegenden Untersuchung auch bei gekreuzter K-Draht-Osteosynthese (Ababneh et al. 1998; Ahmed et al. 2015; Ducić et al. 2016) im prospektiven Kollektiv bei 67\% der Kinder eine sonographisch gemessene Humerustorsionsdifferenz unmittelbar nach Fraktur (sechs Wochen) nachgewiesen werden. Dennoch findet sich bei der Methode mit den gekreuzten K-Drähten eine niedrigere Rate an Rotationsspornen als bei der Methode mit dem radialen Fixateur externe (von Laer 1997). 
Tabelle 47 gibt einen Überblick über die unterschiedlichen Studien und deren Ergebnisse bezüglich OP-Verfahren, Funktionseinschränkungen, Achsfehlstellungen und Rotationssporne.

Tabelle 47: Übersicht über die einzelnen OP-Verfahren und deren Funktionseinschränkungen/ Achsfehlstellungen

\begin{tabular}{|c|c|c|c|c|}
\hline Autor & $\begin{array}{l}\text { Methode/ } \\
\text { Kollektiv }\end{array}$ & $\begin{array}{l}\text { Funktionsein- } \\
\text { schränkungen } \\
\text { (Anzahl) }\end{array}$ & $\begin{array}{l}\text { Achsfehl- } \\
\text { stellungen } \\
\text { (Anzahl) }\end{array}$ & $\begin{array}{l}\text { Rotations- } \\
\text { sporne }\end{array}$ \\
\hline von Laer 1997 & $\begin{array}{l}\text { radialer Fixateur } \\
\text { externe mit } \mathrm{N}= \\
30\end{array}$ & $5(17 \%)$ & 0 & $14(47 \%)$ \\
\hline Gadomski 2017 & $\begin{array}{l}\text { radialer Fixateur } \\
\text { externe mit } \mathrm{N}= \\
22\end{array}$ & $\begin{array}{ll}2(20 \%) & \text { Gruppe } \\
\text { retro } 1 & \text { und } 5 \\
(42 \%) & \text { Gruppe } \\
\text { retro } 2 & \end{array}$ & $\begin{array}{l}3 \quad(30 \%) \text { in } \\
\text { Gruppe retro } 1 \\
\text { und } 2(17 \%) \\
\text { Gruppe retro } 2\end{array}$ & $7(32 \%)$ \\
\hline Scola 2002 & $\begin{array}{l}\text { K-Drähte mit } \\
\mathrm{N}=22\end{array}$ & $1(5 \%)$ & $1(5 \%)$ & \\
\hline Gadomski 2017 & $\begin{array}{l}\text { K-Drähte mit } \\
\mathrm{N}=27\end{array}$ & 0 & $4(15 \%)$ & $5(19 \%)$ \\
\hline $\begin{array}{l}\text { von Bohn et al. } \\
2010\end{array}$ & $\begin{array}{l}\text { K-Drähte mit } \\
\mathrm{N}=178\end{array}$ & $3(2 \%)$ & $\begin{array}{l}8(5 \%) \text { in der } \\
\text { Frontal- und } \\
\text { Sagittalebene }\end{array}$ & \\
\hline
\end{tabular}

\subsubsection{Bedeutung der posttraumatischen Fehlstellung und der Humerustorsion für Funktion und Ellenbogenachse}

Nach suprakondylären Humerusfrakturen kommt es in $10-50 \%$ der Fälle zu einem Cubitus varus (French 1959; Smith 1960; Graham 1967; von Laer 1979; Labelle et al. 1982; Kallio et al. 1992; Gaddy et al. 1994; Hernandez und Roach 1994; Davis et al. 2000; Handelsman et al. 2006; Strohm und Schmittenbecher 2012; von Laer et al. 2013; Hefti 2015). Bei 22 -28\% der Kinder finden sich auch Ellenbogenfunktionseinschränkungen $(28 \%$ bei von Laer 1979, 22\% bei Dallek et al. 1985 und 24\% bei Scola 2002). Der Zeitpunkt der Befunderhebung variiert stark in der Literatur (z. B. NU von ein bis 16 Jahren; von Laer 1979; Dallek et al. 1985; Scola 2002). Im untersuchten prospektiven Kollektiv konnte insbesondere beim sehr frühen Nachuntersuchungszeitpunkt (sechs 
Wochen nach Unfall) festgestellt werden, dass mehr Kinder, nämlich noch fünf (19\%), Funktionseinschränkungen aufwiesen als zum Nachuntersuchungszeitpunkt nach zwölf Monaten (kein Kind). Im retrospektiven Kollektiv Gruppe 1 hatten bei der NU1 noch sechs Kinder (60\%) Funktionseinschränkungen und zwei Kinder $(20 \%)$ keine mehr. In der Gruppe retro 2 hatten zur NU1 noch fünf Kinder (42\%) Funktionseinschränkungen, was sich zur NU3 nicht änderte.

Wie schon eingangs erwähnt, trat im retrospektiven Kollektiv (Gruppe retro 1) ein Torsionsfehler in 100\% der Fälle auf, was aber nur in 60\% der Fälle zu Funktionseinschränkungen und in 30\% zu Achsabweichungen führte. Damit konnte - wie schon bei von Laer (1979) und von Laer et al. (2013) - gezeigt werden, dass trotz vorhandener Torsionsdifferenz keine relevanten funktionellen und kosmetischen (Achse) Einschränkungen zu finden waren.

Im prospektiven Kollektiv hatten bei der Frühuntersuchung sechs Wochen nach Unfall 67\% der Kinder einen Torsionsfehler und alle (100\%) Funktionseinschränkungen sowie zwei Kinder (11\%) eine Achsabweichung. Die Funktionseinschränkungen waren jedoch nach zwölf Monaten nicht mehr vorhanden. Dies ist durch das einsetzende Remodelling des Rotationssporns bedingt. Ein posttraumatischer Rotationssporn führt im Allgemeinen zu einer Einschränkung der Ellenbogenflexion. Trotzdem ist ein direkter unmittelbarer Zusammenhang zwischen der posttraumatischen Torsionsdifferenz und der frühen Ellenbogenfunktionseinschränkung nicht in allen Fällen herzustellen. Dies wurde auch in Untersuchungen von Mahaisavariya und Laupattarakasem (1993) und von Wessel et al. (2003) bestätigt, die keine Korrelation zwischen Rotation der Frakturfragmente, Ellenbogenfunktion und dem Ausmaß der Varusdeformität feststellen konnten.

In der Literatur (Leitlinie suprakonyläre Humerusfraktur beim Kind 2014) werden verbleibende funktionelle Einschränkungen bei einer Varusfehlstellung mit 11,7\% und Valgusfehlstellung mit 7,2\% beschrieben. Der Cubitus varus ist allerdings meistens nur kosmetisch störend und nimmt keinen Einfluss auf die Ellenbogenfunktion (Dowd und Hopcroft 1979; Hernandez und Roach 1994; Strom und Schmittenbecher 2012; von Laer et al. 2013). Das konnte in der vorliegenden Arbeit auch so bestätigt werden.

\subsection{Messung der Humerustorsion}

In der Vergangenheit wurden verschiedene Methoden zur Erfassung der Humerustorsion beschrieben (Gegenbaur 1868; Smith 1960; Graham 1967; Debevoise et al. 1971; Saha 1978; Dowd und Hopcroft 1979; Dähnert und Bernd 1986; Resch und Helweg 1987; Hindman et al. 1988; Söderlund et al. 1989; Fuchs et al. 1991; Harland et al. 1991; Wong und Balasubramaniam 1992; Hernigou et al. 2002). Die klinische Messung der Schulterfunktion (Smith 1960; Graham 1967; Yamamoto et al. 1985) als indirekter Hinweis auf die Humerustorsion hat sich als ungenau erwiesen. Dies konnte auch in den beiden Kollektiven (retrospektives- und prospektives Kollektiv) so nachvollzogen werden mit 
nicht korrelierenden Ergebnissen zwischen Schulterfunktion und Humerustorsionsdifferenz.

Aber auch der Nachweis mittels bildgebender Verfahren ist problematisch. Prinzipiell stehen als bildgebende Verfahren zur Bestimmung der Humerustorsion aktuell die Röntgendiagnostik, das MRT, das CT und die Sonographie zur Verfügung (Smith 1960; Graham 1967; Debevoise et al. 1971; Dowd und Hopcroft 1979; Dähnert und Bernd 1986; Hindman et al. 1988; Söderlund et al. 1989; Fuchs et al. 1991; Harland et al. 1991; Kunz et al. 1993; Ito et al. 1995; Hernigou et al. 2002; Tellioğlu et al. 2014).

Allen Methoden ist gemeinsam, dass eine Standardisierung durch die physiologische Gegebenheit des Humerus erschwert ist. Dies liegt zum einen am Fehlen prägnanter Orientierungspunkte im proximalen Humerusbereich, zum anderen an der dynamischen Entwicklung der Humerustorsion im Wachstumsalter, über die bisher wenig bekannt ist (Krahl 1947). Prinzipiell ist bei der Torsion der proximale Humerus gegen den distalen Anteil gedreht (Debevoise 1971; Harland et al. 1991). Nach Gegenbaur (1868) wird der anatomische Retrotorsionswinkel durch einen Winkel zwischen der proximalen Achse vom Humeruskopfzentrum durch die Mitte des Tuberculum majus und der distalen Achse durch die Epikondylen bestimmt. Im Gegensatz zur Methode der Bestimmung des anatomischen Retrotorsionswinkels (Gegenbaur 1868) orientieren sich beispielsweise computertomographische (Dähnert und Bernd 1986; Hindman et al. 1988; Fuchs et al. 1991; Hernigou et al. 2002) und sonographische (Harland et al. 1991; Kunz et al. 1993; Ito et al. 1995) Methoden nicht an der Kopfhalsachse oder an Hilfslinien zu ihrer Rekonstruktion, sondern am Sulcus intertubercularis und seiner horizontalen Darstellung. Dähnert und Bernd (1986), Harland et al. (1991) und Kunz et al. (1993) konnten nachweisen, dass eine Änderung der Kopfhalsachse mit einer Lageänderung des Sulcus intertubercularis korrelierte. Diese Aspekte sind besonders bei Verfahren wichtig, die den Humeruskopf und den distalen Humerus als Referenzpunkte für die Humerustorsionsbestimmung verwenden, beispielsweise CT, Sonographie und MRT (Tellioğlu et al. 2014).

Exakte radiologische Torsionsmessungen langer Röhrenknochen sind durch die Lagerungsart der Extremität, der Position des Zentralstrahls bzw. dessen Projektionsrichtung mit Vergrößerungsfaktor ungenau (Fuchs et al. 1991). Humerustorsionsmessungen anhand von konventionellen Röntgenbildern (Smith 1960; Graham 1967; Debevoise et al. 1971; Dowd und Hopcroft 1979; Söderlund et al. 1989) haben sich aufgrund von Schwierigkeiten in der Aufnahmetechnik und Lagerung sowie aufgrund von Ungenauigkeit der Messungen nicht durchgesetzt. Falls trotzdem nach einer suprakondylären Humerusfraktur eine Bestimmung von Dislokation und Rotation erfolgen soll, wird im Allgemeinen die Rogers-Hilfslinie eingezeichnet und anhand der Frakturfragmente eine Unterteilung in Extensions- oder Flexionsfraktur mit oder ohne Torsionsfehler vorgenommen. Im frühzeitigen Verlauf nach Unfall kann im seitlichen Röntgenbild ein Rotationssporn als Zeichen eines vorliegenden Torsionsfehlers gesehen werden. Im 
retrospektiven Kollektiv kam es bei insgesamt 32\% der Kinder zu einer Darstellung eines Rotationssporns im Röntgenbild. Im prospektiven Kollektiv bei 19\% der Kinder. In der Literatur bei von Laer (1997) fanden sich sogar 47\% an Rotationsspornen bei der Auswertung radiologischer Bilder. Allerdings bleiben, abhängig vom Strahlengang im seitlichen Röntgenbild, Torsionsfehler bis zu $20^{\circ}$ unerkannt (von Laer et al. 2013). Findet nach Wochen bis Monaten ein Remodelling der Fraktur statt, ist der Rotationssporn radiologisch nicht mehr nachweisbar, sodass dann eine konventionell radiologische Diagnostik zur Erfassung einer Torsionsfehlstellung unmöglich ist (von Laer et al. 2013).

Aufgrund der hohen Strahlenbelastung haben sich computertomographische Methoden zur Torsionsmessung am Humerus bisher nicht etabliert (Dähnert und Bernd 1986; Resch und Helweg 1987; Hindman et al. 1988; Fuchs et al. 1991; Wong und Balasubramaniam 1992; Hernigou et al. 2002). Hierbei beeinträchtigen Abweichungen der Armposition die Präzision der Messergebnisse (Dähnert und Bernd 1986; Fuchs et al. 1991). Computertomographische Messungen haben Schwankungsbreiten der Humerustorsionsnormwerte innerhalb eines Kollektivs von $34-56^{\circ}$ ergeben (Dähnert und Bernd 1986; Fuchs et al. 1991; Wong und Balasubramaniam 1992). Diese Zahlen stimmen mit historischen anatomischen Messungen überein (Gegenbaur 1868; $37^{\circ}$ ). Aufgrund dieser großen Streubreite und der zusätzlich hohen Strahlenbelastung hat sich die CT Untersuchung in der Kindertraumatologie zur Humerustorsionsmessung nicht etabliert und wurde in der vorliegenden Untersuchung bei keinem Kind durchgeführt.

Sonographische Untersuchungen hingegen sind schnell verfügbar, schonend, aussagekräftig und einfach in der Durchführung. Zur Erfassung der Humerustorsion wurden sie erstmals 1987 herangezogen (Harland et al. 1987). In den letzten Jahren haben verschiedene Autoren (Jerosch et al. 1989; Harland et al. 1991; Kunz et al. 1993; Ito et al. 1995) sonographische Methoden zur Humerustorsionsmessung beschrieben. Diese basieren auf sonographischen Darstellungen am Humeruskopf und der Ellenbogenregion. Alle diese Methoden verzichten jedoch auf eine standardisierte Lagerung, was bei Positionsänderung der Armstellung zwischen den beiden Messungen zu Falschwerten führen könnte. Die sonographische Berechnung der Humerustorsion basiert weiterhin auf einer Winkelbildung zwischen sonographischen Schnittebenen am proximalen und distalen Humerus. Bei nicht Darstellbarkeit auf einem Bild oder Verkippung des Schallkopfes ist hier eine weitere Fehlerquelle denkbar.

In der vorliegenden Untersuchung wurde zur Standardisierung der Methodik eine Lagerungsschale mit einem montierten Winkelmessgerät und Wasserwaage angewandt (Katthagen 2008; Katthagen et al. 2009). Ebenfalls wurde nicht der tatsächliche anthropometrische Torsionswinkel erfasst, sondern die Armstellung bei horizontaler Ausrichtung des Sulcus intertubercularis im Ultraschall, wobei der fixierte Unterarm als Zeiger diente. 
Bei der Bestimmung von Messfehlern für sonographische Humerustorsionsmessungen fanden sich Werte von $\pm 7,1$ bis $\pm 11,4 \%$ (Jerosch et al. 1989). Diese lagen höher als in der vorliegenden Untersuchung mit 3,6 ${ }^{\circ}$ (Katthagen et al. 2009). In der vorliegenden Arbeit wurde auch das Vorhandensein eines radiologischen Rotationssporns zur sonographisch gemessenen Humerustorsion korreliert. Erwartungsgemäß fand sich hier wegen der oben beschriebenen Schwierigkeiten der radiologischen Messungen keine 100\%ige Übereinstimmung.

Obwohl die Humerustorsion mit der vorgestellten sonographischen Methode nur indirekt gemessen wurde, zeigte sich eine hohe Reproduzierbarkeit mit einem geringen Inter-Observer-Messfehler von 3,6 ${ }^{\circ}$.

\subsection{Einflussfaktoren auf die Humerustorsion}

Die Vermutung einer physiologischen Veränderung der Humerustorsion im Wachstum, wurde bereits 1947 von dem Anatomen Krahl (Krahl 1947) beschrieben, der die Entwicklung der Humerustorsion studierte. Er nahm an, dass die Humerustorsion nahe der proximalen Epiphyse stattfinde und die sekundäre Humerustorsion nach der Geburt aufgrund der dynamischen Kräfte der Schultermuskulatur einsetze. Dies wurde auch aufgrund anatomischer Studien und Analysen von Muskelwirkungen von von Lanz und Wachsmuth (1935) vermutet. Krahl (1947) beobachtete eine Sekundärtorsion von Ø $32^{\circ}$ und eine lineare Korrelation zwischen Torsionswinkel und zunehmendem Alter von Geburt bis zum Wachstumsabschluss. Ito et al. (1995) kalkulierten eine Zunahme der Humerustorsion von $14^{\circ}$ zwischen Geburt und Wachstumsabschluss, allerdings bei sehr kleinen Fallzahlen. Um diesem Phänomen auch in der vorliegenden Arbeit Rechnung zu tragen, wurden Normwerte für sonographisch gemessene Humerustorsion in einem gesunden Kollektiv ermittelt (Katthagen et al. 2009). In der Querschnittsuntersuchung des KinderNormalkollektivs, wo beide oberen Extremitäten gemessen wurden, fand sich ebenfalls eine Zunahme der Humerustorsion mit steigendem Alter, wobei hier jedoch einschränkend keine Daten von Säuglingen erfasst wurden. Bei Krahl (1947) fand sich stets eine Innentorsion, was in unserem Kollektiv auch überwiegend bei $84 \%$ der Kinder vorlag.

Im hier untersuchten prospektiven Kollektiv mit vorhandener Humerustorsionsdifferenz fand sich am gesunden Arm eine physiologische Zunahme der Humerustorsion. Der frakturierte Arm zeigte als posttraumatische Folge eine erhöhte Humerustorsion im Sinne einer erhöhten Innentorsion. Dieser Wert führte zu einer langsameren physiologischen Humerustorsionsänderung, sodass sich beide Werte im Verlauf wieder annäherten, also eine signifikante Verminderung der Differenz stattfand. Somit zeigte sich in dem untersuchten Kollektiv auch die für die untere Extremität beschriebene Annäherung der posttraumatischen Torsionsdifferenz. Dies wurde 1961 von Morscher für Femurtorsionsdifferenzen im Verlauf des Wachstums beschrieben (Morscher 1961). 
Am Femur ist bekannt, dass durch funktionelle Kräfte am proximalen Femur posttraumatische Torsionsfehler bis zum Abschluss des Wachstums ein Remodellierungspotential aufweisen (Morscher 1961; Zukunft-Huber 2011). Ursächlich ist hierfür wahrscheinlich der funktionelle Anreiz beim Gehen, also das Geradestellen des Fußes. Bei neurologischen Krankheitsbildern kann diese Verminderung der Antetorsion ausbleiben (Zukunft- Huber 2011), und es wurde sogar eine erneute Zunahme der femoralen Antetorsion nach operativer Korrektur bei Innenrotationsfehlstellungen bei Kindern mit spastischer Hemiparese beschrieben (Morscher 1961). Ähnliche funktionelle Korrekturmechanismen sind auch für die obere Extremität, allerdings in geringerem Ausmaß denkbar.

Einige Autoren (Ariño et al. 1977; Vahvanen und Aalto 1978; von Laer et al. 2013) spekulieren, dass eine Spontankorrektur des Torsionsfehlers am Oberarm zumindest theoretisch über physiologische Detorsionsvorgänge möglich ist, während andere wiederum rein theoretisch solche Korrekturen grundsätzlich ausschließen (Cramer et al. 1992).

Prinzipiell wurden an der oberen Extremität Humerustorsionsdifferenzen bei Kindern mit spastischer Hemiparese festgestellt (Katthagen 2008). Das Ausmaß der Humerustorsionsdifferenz korrelierte hier mit dem Grad der Spastik.

In Untersuchungen an professionellen Sportlern (Pieper 1982; Bass et al. 2002; Osbahr et al. 2002; Mair et al. 2004; Schmidt-Wiethoff et al. 2004) konnte gezeigt werden, dass bei Wurfsportarten wie zum Beispiel Handball, Basketball oder beim Tennis der dominante Arm eine vermehrte Humerusaußentorsion aufweist bei verminderter Innenrotationsfähigkeit der Schulter im Vergleich zur Gegenseite. Somit scheinen am wachsenden Skelett Torsionsvorgänge am Humerus altersabhängig und belastungsabhängig zu sein (Bass et al. 2002). Die nachgewiesene Beeinflussung der Humerustorsion durch externe Faktoren (z. B. Leistungssport) wurde in der vorliegenden Untersuchung nicht erfasst. Allerdings wurde zur Beeinflussung der täglichen Belastung die Händigkeit der Kinder untersucht. Hier konnte im prospektiven Kollektiv kein Einfluss der Händigkeit auf die Humerustorsionsentwicklung festgestellt werden, was auch Ito et al. (1995) bestätigten.

\subsection{Korrektur der Torsionsdifferenz nach suprakondylären Humerusfrakturen im Kindesalter}

Nach Analyse der Daten konnte im vorliegenden prospektiven Kollektiv erstmals nachgewiesen werden, das trotz vorhandener Torsionsdifferenz nach suprakondylären Humerusfrakturen im Kindesalter eine Spontankorrektur stattfand. Von den 67\% der Kinder mit sonographisch gemessener Humerustorsionsdifferenz zeigte sich eine durchschnittliche Verminderung von $14^{\circ}$ (sechs Wochen) auf 7,8 $8^{\circ}$ (vier Monate) auf 6,5 (sechs Monate) und auf 4,9 (zwölf Monate). Im prospektiv evaluierten Kollektiv ( $\mathrm{n}=18$ ) mit nachweislich vorhandener Differenz glich sich diese im Verlauf der Untersuchung in 
Abhängigkeit vom Alter wieder an, nicht nur durch eine aktive Detorsion, sondern auch durch das „Aufholen“ im Wachstum der unverletzten Extremität.

Im retrospektiven Kollektiv mit längeren Zeiten nach dem Unfall zeigten sich ebenfalls noch Veränderungen der Humerustorsionsdifferenzen, die aber ausgeprägter im ersten Jahr nach Unfall waren als danach. Diese waren jedoch nicht signifikant. Weiterhin wurde festgestellt, dass kleine Kinder ( $<$ zehn Jahre) besser korrigierten als ältere Kinder. Ein Einfluss des Alters auf Korrekturvorgänge wurde bereits von von Laer et al. (2013) postuliert.

Der Zeitraum der Korrekturen und das Alter lassen auf eine Mischung aus einem funktionell-mechanischen Korrekturmechanismus, wie dies auch zum Beispiel bei der Spontankorrektur der dislozierten proximalen Radiusfraktur bekannt ist (Vocke und von Laer 1998; von Laer et al. 2013), in Kombination mit einer Korrektur durch Wachstum schließen. Hierzu wäre eine Analyse posttraumatischer Humerustorsionsdifferenzen beim Erwachsenen zur Differenzierung dieses Mechanismus interessant.

\subsection{Fazit, Limitationen der Untersuchung und Ausblick}

In der vorliegenden Arbeit wurde erstmals an einem prospektiven Kollektiv von Kindern mit dislozierten suprakondylären Humerusfrakturen die Spontankorrektur der Humerustorsionsdifferenz im zeitlichen Verlauf beschrieben. Durch die zusätzliche Analyse eines heterogenen retrospektiven Kollektivs mit großen Humerustorsionsdifferenzen - bedingt durch die OP-Methode mit dem radialen Fixateur externe nach von Laer (von Laer 1997) konnte der Zeitraum der größten Veränderung auf die ersten zwölf Monate nach Unfall eingegrenzt werden. Kleinere Kinder zeigten ein größeres Korrekturpotential als ältere Kinder. Die Händigkeit hatte keinen Einfluss.

Wie oben bereits ausführlich erläutert, ist die Diagnose einer Humersutorsionsdifferenz schwierig. Selbst die hier vorgestellte verbesserte standardisierte sonographische Methode stellt keine routinemäßige Untersuchung dar. Eine vergleichende Anwendung dieser Messmethode in anderen Zentren erfolgte daher bisher nicht.

Die Werte der Humerustorsion auf der gesunden und frakturierten Seite, aber auch im Normalkollektiv weisen eine große Streubreite auf (Abbildung 25). Somit besteht anscheinend eine große individuelle Varianz.

Ob sportliche Aktivität bzw. Muskeltraining die Detorsion nach Oberarmbrüchen, speziell an der oberen Extremität, noch weiter beeinflussen kann, sollte in weiteren Arbeiten geklärt werden.

Da beide Kollektive recht kleine Fallzahlen aufwiesen, wäre eine statistische Analyse mit mehr Kindern mit operativ versorgten suprakondylären Humerusfrakturen noch aussagekräftiger. 


\section{$5 \quad$ Zusammenfassung}

Nach suprakondylären Humerusfrakturen im Wachstumsalter ist die Bedeutung des posttraumatischen Humerustorsionsfehlers umstritten. Während die einen ihn ausnahmslos für den kosmetisch störenden Cubitus varus verantwortlich machen, sehen die anderen ihn als Ursache für ein sekundäres mediales Abkippen, das dann zum Cubitus varus führen kann. Ebenso ist eine klinische oder bildgebende Erfassung schwierig.

Zur Diagnostik des Torsionsfehlers am Humerus wurde eine einfache standardisierte sonographische Methode zur Humerustorsionsmessung angewandt. Parallel wurden wiederholte anamnestische, klinische und radiologische Daten erfasst und ausgewertet. Zur Beurteilung des Langzeitverlaufes einer posttraumatischen Humerustorsionsdifferenz wurde ein retrospektiven Kollektiv $(\mathrm{N}=22)$ analysiert, welches mit einem radialen Fixateur externe versorgt worden war. Eine Gegenüberstellung zu einem prospektiven Kollektiv $(\mathrm{N}=27)$, welches mittels perkutaner gekreuzter K-Draht-Osteosynthese versorgt worden war, erfolgte in standardisierten Untersuchungsabständen (sechs Wochen, vier Monate, sechs Monate, zwölf Monate). Statistische Analysen wurden durchgeführt.

Im prospektiven Kollektiv fand sich eine Humerustorsionsdifferenz bei 67\% der Kinder sechs Wochen nach Unfall. Eine durchschnittliche Verminderung von $14^{\circ}$ (sechs Wochen) auf $7,8^{\circ}$ (vier Monate) auf $6,5^{\circ}$ (sechs Monate) und auf 4,9 ${ }^{\circ}$ (zwölf Monate) fand im Verlauf statt. Diese war bei kleinen Kindern unter zehn Jahren höher als bei älteren. Eine erhöhte Innentorsion $\geq 20^{\circ}$ wurde in allen Kollektiven am besten korrigiert $\left(13,4^{\circ}\right.$ Gruppe retro $1,8,8^{\circ}$ Gruppe retro 2 und 11,0 ${ }^{\circ}$ im prospektiven Kollektiv). Im retrospektiven Kollektiv hatte die Mehrzahl der Kinder (100\% im ersten Jahr und 75\% > ein Jahr nach Unfall) eine Humerustorsionsdifferenz, welche sich im ersten Jahr nach Unfall mehr korrigierte als danach. Bleibende funktionelle und kosmetische Einschränkungen fanden sich im retrospektiven Kollektiv Gruppe retro 1 bei 20\%, in der Gruppe retro 2 bei $60 \%$ und im prospektiven Kollektiv keine. Bei der Analyse eines Normalkollektivs konnte die physiologische Zunahme der Humerusinnentorsion durch sonographische Messungen erneut bestätigt werden, ebenso wie eine nicht signifikante Differenz der Extremitäten untereinander. Die standardisierte sonographische Methode zeigte einen Inter-Observer-Messfehler von 3,6 ${ }^{\circ}$.

In der vorliegenden Arbeit konnte erstmals nachgewiesen werden, dass nach posttraumatischen Torsionsfehlern am Humerus Detorsionsvorgänge im Verlauf am wachsenden Skelett stattfinden. Diese fanden sich verstärkt im ersten Jahr nach Unfall, bei starkem Innentorsionsfehler und bei kleinen Kindern. Jenseits des ersten Jahres nach Unfall (Gruppe retro 2) fanden nur noch unzureichende Korrekturen statt. Kinder mit posttraumatischer Humerustorsionsdifferenz im prospektiven Kollektiv zeigten altersabhängig eine Annäherung an den physiologischen Normalwert. 


\section{$6 \quad$ Literaturverzeichnis}

Ababneh M, Shannak A, Agabi S, Hadidi S (1998): The treatment of displaced supracondylar fractures of the humerus in children. Int Orthop $\underline{22}$, 263-265

Ahmed W, Rasheed H, Mohib Y, Rashid RH (2015): K Wire fixation of supra-condylar humerus fractures in children. Is ulnar nerve at risk? J Pak Med Assoc 65, 202-204

Aitken PA, Smith L, Blackett CW (1943): Supracondylar fractures in children Am J Surg 59(2), 161 171

Ariño VL, Lluch EE, Ramirez AM, Ferrer J, Rodriguez L, Baixauli F (1977): Percutaneous fixation of supracondylar fractures of the humerus in children. J Bone Joint Surg Am $\underline{59}$, 914-916

Arnold JA, Nasca RJ, Nelson CL (1977): Supracondylar Fractures of the Humerus: The Role of Dynamic Factors in the Prevention of Deformity. J Bone Joint Surg 59-A(5), 589-595

AWMF- Leitlinie (2014): Suprakondyläre Humerusfraktur beim Kind. Aktueller Stand 12/2014; Online-Publikation http://www.awmf.org/uploads/tx szleitlinien/0120141 Suprakondyl $\%$ C3\%A4re Humerusfraktur Kind-2014-12.pdf

Aumüller G, Aust G, Engele J, Kirsch J, Maio G, Mayerhofer A, Mense S, Reißig D, Salvetter J, Schmidt W, et al.: Duale Reihe Anatomie. 3. Auflage; Georg Thieme Verlag, Stuttgart 2014

Baierlein SA: Frakturklassifikationen. Georg Thieme Verlag, Stuttgart 2011

Bass SL, Saxon L, Daly RM, Turner CH, Robling AG, Seeman E, Stuckey S (2002): The effect of mechanical loading on the size and shape of bone in pre-, peri-, and postpubertal girls: a study in tennis players. J Bone Miner Res $\underline{17}$, 2274-2280

Baumann E (1960): Zur Behandlung der Knochenbrüche am Ellbogengelenk. Langenbecks Arch Klin Chir 295, 300-304

Benz-Bohm G: Kinderradiologie. Georg Thieme Verlag, Stuttgart 2005

Blakey CM, Biant LC, Birch R (2009): Ischaemia and the pink, pulseless hand complicating supracondylar fractures of the humerus in childhood: long-term follow-up. J Bone Joint Surg Br 1ㅗ, 1487-1492

Blount WP: Knochenbrüche bei Kindern. Dt. Übersetzung von K.H. Müller. Thieme Verlag, Stuttgart 1957

Bohn B, Beck O, Werbeck R (2010): Konservative und operative Therapie der suprakondylären Humerusfraktur im Kindesalter. Obere Extrem $\underline{5}, 143-150$

Brauer CA, Lee BM, Bae DS, Waters PM, Kocher MS (2007): A systematic review of medial and lateral entry pinning versus lateral entry pinning for supracondylar fractures of the humerus. J Pediatr Orthop 27, 181-186

Broca P (1881): La torsion de l'humerus et le topomètre, instrument destiné à mesurer la torsion des os (rédigé par L Mamouvier). Rev Anthrop 2(4): 193-210, 385-423 
Brossmann J, Czerny C, Freyschmidt J: Freyschmidt`s "Köhler/Zimmer" Grenzen des Normalen und Anfänge des Pathologischen in der Radiologie des kindlichen und erwachsenen Skeletts. 14. Auflage; Georg Thieme Verlag, Stuttgart 2000

Brouwer KJ (1981): Torsional deformities after fractures of the femoral shaft in childhood. A retrospective study, 27-32 years after trauma. Acta Orthop Scand Suppl 195, 1-167

Buchholz IM, Bolhuis HW, Bröker FHL, Gratama JWC, Sakkers RJB, Bouma WH (2002): Overgrowth and Correction of Rotational Deformity in 12 Femoral Shaft Fractures in 3-6Year-Old Children Treated with an External Fixator. Acta Orthop Scand 73(2): 170-174

Chess DG, Leahey JL, Hyndman JC (1994): Cubitus varus: significant factors. J Pediatr Orthop 14, 190-192

Corres-Franco IA, Torres-Gómez A (2015): Classification of pediatric supracondylar fractures: Gartland, AO and Holmberg: Which has the best interobserver agreement? Acta Ortop Mex $\underline{29}, 299-302$

Cramer KE, Devito DP, Green NE (1992): Comparison of closed reduction and percutaneous pinning versus open reduction and percutaneous pinning in displaced supracondylar fractures of the humerus in children. J Orthop Trauma $\underline{6}, 407-412$

Dähnert W, Bernd W (1986): Computertomographische Bestimmung des Torsionswinkels am Humerus. Z Orthop Unfallchir 124, 46-49

Dallek M, Mommsen U, Jungbluth KH, Kahl HJ (1985): Die suprakondyläre Humerusfraktur im Kindesalter, ihre Behandlung und Ergebnisse nach der Methode von Blount. Unfallchirurgie $\underline{11}, 192-196$

Davis RT, Gorczyca JT, Pugh K (2000): Supracondylar humerus fractures in children. Comparison of operative treatment methods. Clin Orthop $\underline{346}, 49-55$

Debevoise NT, Hyatt GW, Townsend GB (1971): Humeral torsion in recurrent shoulder dislocations. A technic of determination by X-ray. Clin Orthop $\underline{76}$, 87-93

De las Heras J, Durán D, de la Cerda J, Romanillos O, Martínez-Miranda J, Rodriguez-Merchán EC (2005): Supracondylar Fractures of the Humerus in Children. Clin Orthop 432, 57-64

Dowd GS, Hopcroft PW (1979): Varus deformity in supracondylar fractures of the humerus in children. Injury $\underline{10}, 297-303$

Ducić S, Bumbasirević M, Radlović V, Nikić P, Bukumirić Z, Brdar R, Radojicić Z, Bukva B, Abramović D, Ducić TJ (2016): Displaced supracondylar humeral fractures in children: Comparison of three treatment approaches. Srp Arh Celok Lek 144, 46-51

Felsenreich F (1931): Kindliche suprakondyläre Frakturen und posttraumatische Deformitäten des Ellbogengelenkes. Arch Orthop Unfallchir 29 , 555-578

Feng C, Guo Y, Zhu Z, Zhang J, Wang Y (2012): Biomechanical analysis of supracondylar humerus fracture pinning for fractures with coronal lateral obliquity. J Pediatr Orthop $\underline{32}$, 196-200

Fernandez FF, Eberhardt O (2012): Suprakondyläre Humerusfraktur bei Kindern: Verfahrenswahl, Zugang, Komplikationen und typische Fallstricke. Trauma Berufskrankh 14(3), 379-384 
Fernandez FF, Eberhardt O, Wirth T (2016): Suprakondyläre Humerusfrakturen im Wachstumsalter. OUP $\underline{5}(3), 140-145$

Field A: Discovering Statistics using SPSS. 3. Auflage; SAGE Publications, Los Angeles 2009

Flynn JC, Matthews JG, Benoit RL (1974): Blind Pinning of Displaced Supracondylar Fractures of the Humerus in Children. J Bone Joint Surg Am 56(2), 263- 272

France J, Strong M (1992): Deformity and function in supracondylar fractures of the humerus in children variously treated by closed reduction and splinting, traction, and percutaneous pinning. J Pediatr Orthop $\underline{12}$, 494-498

French PR (1959): Varus deformity oft he ellbow follwoing supracondylar fractures oft he humerus in children. Lancet 2 , 439-41

Frick H, Leonhardt H, Starck D: Allgemeine Anatomie. Spezielle Anatomie: 1. ExtremitätenRumpfwand- Kopf-Hals. Georg Thieme Verlag, Stuttgart 1987

Fuchs C, Schmid P, Engelhardt P (1991): Computertomographische Humerustorsionsmessung. Z Orthop Unfallchir $\underline{129}$, 423-425

Gaddy BC, Manske PR, Pruitt DL, Schoenecker PL, Rouse AM (1994): Distal Humeral Osteotomy for Correction of Posttraumatic Cubitus Varus. J Pediatr Orthop 14, 214-219

Gartland JJ (1959): Management of supracondylar fractures of the humerus in children. Surg Gynecol Obstet 109, 145-154

Gegenbaur C (1868): Über die Drehung des Humerus. Jenaische Z für Med Naturwissenschaft $\underline{4}$, $50-63$

Gercek E, Nusselt T, Rothenbach E, Hartmann F (2015): Suprakondyläre Humerusfrakturen im Wachstumsalter: Operative Schwierigkeiten und mögliche Konsequenzen im Verlauf. Trauma Berufskrankh 17, 260-264

Graham H (1967): Supracondylar fractures of the elbow in children.Part 1 and Part 2. Clin Orthop $\underline{54}, 85-102$

Handelsman JE, Weinberg J, Hersch JC (2006): Corrective supracondylar humeral osteotomies using the small AO external fixator: J Pediatr Orthop B $\underline{15}$, 194-197

Harland U, Diepolder M, Gruber G, Knöss H-P (1991): Die sonographische Bestimmung des Humerusretrotorsionswinkels. Z Orthop Unfallchir $\underline{129}$, 36-41

Hasler CC (2001): Supracondylar Fractures of the Humerus in Children: Eur J Trauma 27, 1-15

Hefti F: Kinderorthopädie in der Praxis. 3., vollst. überarb. Auflage; Springer Verlag, Berlin 2015

Hernandez MA, Roach JW (1994): Corrective Osteotomy for Cubitus Varus Deformity: J Pediatr Orthop 14, 487-491

Hernigou P, Duparc F, Hernigou A (2002): Determining Humeral Retroversion with Computed Tomography. J Bone Jt Surg $\underline{84}$, 1753-1762

Hindman BW, Schreiber RR, Wiss DA, Ghilarducci MJ, Avolio RE (1988): Supracondylar fractures of the humerus: prediction of the cubitus varus deformity with CT. Radiology $\underline{168}, 513-515$ 
Holmberg L (1945): Fractures in the distal end of the humerus in children. Acta Chir Scand 143 , 1-69

Hultkrantz JW: "Torsion" des Oberarmkopfes. In: Fick R, Eisler P, von Bardeleben (Hrsg): Handbuch der Anatomie des Menschen, Bd. 2. G. Fischer, Jena 1904, 164-168

Imhoff AB, Linke RD: Gelenkspezifische Untersuchungstechniken. In: Imhoff AB, Linke RD, Baumgartner R, Ahrens P (Hrsg.): Checkliste Orthopädie. 2., komplett überarb. und erw. Auflage; Thieme Verlag, Stuttgart 2011, 40

Ito N, Eto M, Maeda K, Rabbi M, Iwasaki K (1995): Ultrasonographic measurement of humeral torsion. J Shoulder Elbow Surg 4, 157-161

Jerosch J, Sons H, Sterken W, Winkelmann W (1989): Die sonographische Bestimmung des Humerus-Retrotorsionswinkels. Ultraschall Med 10, 270-274

Jorden N: Distale Humerusfrakturen. In: Bohndorf K, Imhof H, Wörtler K (Hrsg.): Radiologische Diagnostik der Knochen und Gelenke. 3. Auflage; Georg Thieme Verlag, Stuttgart 2014, 143

Kaiser MM, Kamphaus A, Massalme E, Wessel LM (2008): Gekreuzte Kirschner-DrahtOsteosynthese der suprakondylären Humerusfraktur bei Kindern. Oper Orthop Traumatol 20 , 297-309

Kallio PE, Foster BK, Paterson DC (1992): Difficult supracondylar elbow fractures in children: analysis of percutaneous pinning technique. J Pediatr Orthop $\underline{12}$, 11-15

Kao H-K, Lee W-C, Yang W-E, Chang C-H (2016): Clinical significance of anterior humeral line in supracondylar humeral fractures in Children. Injury 47, 2252-2257

Katthagen JC: Humerustorsion bei Kindern und Jugendlichen mit spastischer Hemiparese. Med. Diss. Göttingen 2008

Katthagen JC, Rudolph C, Hell AK (2009): Sonographische Humerustorsionsmessungen bei

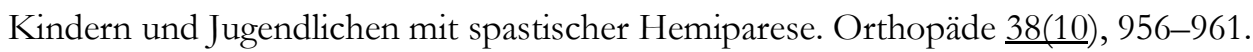

Kocher MS, Kasser JR, Waters PM, Bae D, Snyder BD, Hresko MT, Hedequist D, Karlin L, Kim Y-J, Murray MM (2007): Lateral entry compared with medial and lateral entry pin fixation for completely displaced supracondylar humeral fractures in children. A randomized clinical trial. J Bone Joint Surg Am 오, 706-712

Krahl VE (1947): The torsion of the humerus: Its localization, cause and duration in man. Am J Anat $\underline{80(3)}, 275-319$

Kraus R, Schneidmüller, D, Röder, C (2005): Häufigkeit von Frakturen der langen Röhrenknochen im Wachstumsalter. Dtsch Ärztebl 102 (12A), 838-842

Kraus R, Berthold LD, Laer L von (2007): Effiziente Bildgebung von Ellenbogenverletzungen bei Kindern und Jugendlichen. Klin Pädiatr 219, 282-287

Kraus T, Tauber S, Linhart W (2013): Posttraumatische Komplikationen am kindlichen Ellenbogen. Orthop $\underline{42(1)}, 57-70$

Kubinger KD, Rasch D, Yanagida T: Statistik in der Psychologie: Vom Einführungskurs bis zur Dissertation. Hogrefe Verlag, Göttingen 2011 
Kumar R, Kiran EK, Malhotra R, Bhan S (2002): Surgical management of the severely displaced supracondylar fracture of the humerus in children. Injury $\underline{33}, 517-522$

Kunz C, Rieder T, Viehweger R (1993): Ist die Sonographie zur Torsionswinkelmessung am Humerus einsetzbar? Z Für Orthop Unfallchirurgie 131, 307-312

Labelle H, Bunnell WP, Duhaime M, Poitras B (1982): Cubitus varus deformity following supracondylar fractures of the humerus in children. J Pediatr Orthop 2, 539-546

Lacher M, Schaeffer K, Boehm R, Dietz HG (2011): The treatment of Supracondylar Humeral Fractures With Elastic Stable Intramedullary Nailing (ESIN) in Children. J Pediatr Orthop $\underline{31(1)}, 33-38$

Leitlinie Suprakondyläre Humerusfraktur beim Kind s. AWMF- Leitlinie 2014

Lubinus (1924): über den Entstehungsmechanismus und die Therapie der suprakondylären Humerusfraktur. Dtsch Z Für Chir 186, 289-350

Lutz N, Audigé L, Schmittenbecher P, Clavert J-M, Frick S, Slongo T (2011): Diagnostic algorithm for a validated displacement grading of pediatric supracondylar fractures. J Pediatr Orthop $\underline{31}$, $117-123$

Mahaisavariya B, Laupattarakasem W (1993): Supracondylar fracture of the humerus: malrotation versus cubitus varus deformity. Injury $24,416-418$

Mair SD, Uhl TL, Robbe RG, Brindle KA (2004): Physeal changes and range-of-motion differences in the dominant shoulders of skeletally immature baseball players. J Shoulder Elbow Surg $\underline{13}$, $487-491$

Mann TS (1963): Prognosis in supracondylar fractures. The Journal of bone and Joint Surgery $\underline{45}$, 516-522

Mitsunari A, Muneshige H, Ikuta Y, Murakami T (1995): Internal rotation deformity and tardy ulnar nerve palsy after supracondylar humeral fracture. J Shoulder Elbow Surg 4, 23-29

Morscher E (1961): Die mechanischen Verhältnisse des Hüftgelenkes und ihre Beziehungen zum Halsschaftwinkel und insbesondere zur Antetorsion des Schenkelhalses während der Entwicklungsjahre. Z Für Orthop Ihre Grenzgeb 쏘, 374-394

Niethard F, Pfeil J: Duale Reihe Orthopädie. 5. Auflage; Georg Thieme Verlag, Stuttgart 2005

Niethard FU: Kinderorthopädie. 2., aktualisierte und erw. Auflage; Georg Thieme Verlag, Stuttgart 2010

Oberhammer J (1980): Degree and frequency of rotational deformities after infant femoral fractures and their spontaneous correction. Arch Orthop Trauma Surg 97, 249-255

Osbahr DC, Cannon DL, Speer KP (2002): Retroversion of the humerus in the throwing shoulder of college baseball pitchers. Am J Sports Med $\underline{30}, 347-353$

Pan Z, Chen J, Qu L, Cui Y, Sun C, Zhang H, Yang X, Guan Q (2015): Correlation between Anatomical Parameters of Intertubercular Sulcus and Retroversion Angle of Humeral Head. Int J Clin Exp Med 8(4), 4837-4845 
Pieper H: Untersuchungen zur Torsion des Humerus. Röntgenologische Bestimmung am Patienten und Bedeutung bei den habituellen Schulterluxationen. Med. Diss. Marburg 1982

Pretell-Mazzini J, Rodriguez-Martin J, Andres-Esteban EM (2010): Does open reduction and pinning affect outcome in severely displaced supracondylar humeral fractures in children? A systematic review. Strateg Trauma Limb Reconstr $\underline{5}, 57-64$

Resch H, Helweg G (1987): Die Bedeutung des Rotationsfehlers bei der suprakondylären Oberarmfraktur des Kindes. Akt Traumatol 17, 65-72

Rogers LF, Malave S, White H, Tachdjian MO (1978): Plastic bowing, torus and greenstick supracondylar fractures of the humerus: radiographic clues to obscure fractures of the elbow in children. Radiology 128, 145-150

Saha AK: Rezidivierende Schulterluxationen. Ferdinand EnkeVerlag, Stuttgart 1978

Schäffer K, Böhm R, Dietz HG (2007): Die elastisch-stabile intramedulläre Nagelung (ESIN) der suprakondylären Humerusfraktur im Kindesalter. Unfallchirurg 110, 852-858

Scharizer E: Die Entwicklung der Modernen Unfallchirurgie. Ein Mediko-Historischer Überblick. Springer Verlag, Berlin 1964

Schlickewei W, Seif El Nasr M, Weinberg AM: Diaphysärer Oberschenkel . In: Weinberg AM und Tscherne H (Hrsg.): Unfallchirurgie im Kindesalter. Springer Verlag, Berlin 2006, 637

Schmidt-Wiethoff R, Rapp W, Mauch F, Schneider T, Appell HJ (2004): Shoulder Rotation Characteristics in Professional Tennis Players. Int J Sports Med 25, 154-158

Schofer M, Jung W, Hunger N, Kortmann H-R (2003): Kindliche suprakondyläre Oberarmfrakturen. Trauma Berufskrankh $\underline{5}, 6-12$

Schünke M, Schulte E, Schumacher U: PROMETHEUS LernAtlas der Anatomie: Allgemeine Anatomie und Bewegungssystem. 3. Auflage; Thieme Verlag, Stuttgart 2011

Scola E, Jezussek D, Kerling HP, Yedibela S (2002): Die dislozierte suprakondyläre Humerusfraktur des Kindes. Operationstechnik und Ergebnisse mit dem dorsolateralen Zugang. Unfallchirurg 105(2), 95-98.

Siebert HR, Pannike A (1984): Kriterien zur Behandlung von Oberschenkelschaftfrakturen im Kindesalter. Unfallchirurg 10(1), 45-50

Slongo T, Audigé L (2007): Fracture and Dislocation Classification. Compendium for Children. J Orthop Trauma 21, 135-160

Slongo T, Schmid T, Wilkins K, Joeris A (2008): Lateral external fixation--a new surgical technique for displaced unreducible supracondylar humeral fractures in children. J Bone Joint Surg Am 90, $1690-1697$

Smith L (1960): Deformity Following Supracondylar Fractures of the Humerus. J Bone Jt Surg $\underline{42(2)}, 235-252$

Söderlund V, Kronberg M, Broström LA (1989): Radiologic assessment of humeral head retroversion. Description of a new method. Acta Radiol 1987 30, 501-505 
Sommerfeldt DW (2013): Gelenknahe Frakturen der oberen Extremität im Kindes- und Jugendalter. Trauma Berufskrankh 15, 59-66

Spyra V: Spontankorrektur von pathologischen posttraumatischen Antetorsionen. Med. Diss. Ulm 2011

Steinbrück I, Baumhoer D, Henle P: Intensivkurs Anatomie. Urban \& Fischer, München 2008

Strohm PC, Schmittenbecher PP (2012): Cubitus varus/valgus nach Frakturen des distalen Humerus: Indikationen zur Korrekturosteotomie. Trauma Berufskrankh 14(S3), 385-87

Takeyasu Y, Murase T, Miyake J, Oka K, Arimitsu S, Moritomo H, Sugamoto K, Yoshikawa H (2011): Three-dimensional analysis of cubitus varus deformity after supracondylar fractures of the humerus. J Shoulder Elbow Surg 20, 440-448

Tellioğlu AM, Karakaş S, Taşkin F (2014): Determining Torsion Angle of Humerus Head Using MRI Method. Turk J Med Sci 44(4), 639-642

Vahvanen V, Aalto K (1978): Supracondylar fracture of the humerus in children. A long-term follow-up study of 107 cases. Acta Orthop Scand 49, 225-233

Verbeek HO (1979): Does rotation deformity, following femur shaft fracture, correct during growth? Reconstr Surg Traumatol 17, 75-81

Vocke AK, von Laer L (1998): Die Prognose proximaler Radiusfrakturen im Wachstumsalter. Unfallchirurg 101, 287-295

von Laer L (1977): Overgrowth (of the femoral shaft) and rotational deformities following femoral shaft fractures in childhood. Arch Orthop Unfallchir $\underline{\text { 89(2), 121-137 }}$

von Laer L (1979): Die supracondyläre Humerusfraktur im Kindesalter. Arch Orthop Trauma Surg $\underline{95}, 123-140$

von Laer L, Hasler CC (2000): Mechanical Dynamics in Supracondylar Fractures of the Humerus in Children. LWW 15(1), 30-37

von Laer L, Günter SM, Knopf S, Weinberg AM (2002): Die suprakondyläre Oberarmfraktur im Kindesalter - eine Effizienzstudie Ergebnisse der multizentrischen Studie der Sektion Kindertraumatologie der Deutschen Gesellschaft für Unfallchirurgie - Teil II: Aufwand und Nutzen der Behandlung. Unfallchirurg 105, 217-223

von Laer L, Kraus R, Linhart WE: Frakturen und Luxationen im Wachstumsalter. 6., überarb. und erw. Auflage; Thieme Verlag, Stuttgart 2013

von Laer LR (1997): Der radiale Fixateur externe zur Behandlung suprakondylärer Humerusfrakturen im Wachstumsalter. Operat Orthop Traumatol 9, 265-276

von Lanz T, Wachsmuth W: Praktische Anatomie. Ein Lehr- und Hilfsbuch der anatomischen Grundlagen ärztlichen Handelns. Erster Band/ Dritter Teil: Arm; Julius Springer, Berlin 1935

von Salis-Soglio GF: Die Neutral-0-Methode. Springer Verlag, Berlin 2015

Wang X, Feng C, Wan S, Bian Z, Zhang J, Song M, Shao J, Yang X (2012): Biomechanical analysis of pinning configurations for a supracondylar humerus fracture with coronal medial obliquity. J Pediatr Orthop Part B 21, 495-498 
Weinberg A-M, Marzi I, Günter SM, Wessel L, Riedel J, Laer L v (2002): Die suprakondyläre Oberarmfraktur im Kindesalter - eine Effizienzstudie Ergebnisse der multizentrischen Studie der Sektion Kindertraumatologieder Deutschen Gesellschaft für Unfallchirurgie - Teil I: Epidemiologie, Effektivitätsprüfung und Klassifikation. Unfallchirurg 105, 208-216

Weise K, Schwab E, Scheufele TM (1997): Ellenbogenverletzungen im Kindesalter. Unfallchirurg $\underline{100}, 255-269$

Wessel LM: Suprakondyläre Humerusfraktur. In: Marzi I (Hrsg): Kindertraumatologie. 2. Auflage; Springer, Heidelberg 2010, 143-156

Wessel LM, Günter SM, Jablonski M, Sinnig M, Weinberg AM (2003): Wie lässt sich die Wachstumsprognose nach kindlicher suprakondylärer Humerusfraktur erfassen? Orthop $\underline{32}$, 824-832

White L, Mehlman CT, Crawford AH (2010): Perfused, pulseless, and puzzling: a systematic review of vascular injuries in pediatric supracondylar humerus fractures and results of a POSNA questionnaire. J Pediatr Orthop $\underline{30}$, 328-335

Wilkins K: Supracondylar fractures of the distal Humerus. In: Rockwood C, Wilkins K, Beaty J (Hrsg.): Fractures in children. Lippincott Williams \& Wilkins, Baltimore 1996, 669-750

Wong H, Balasubramaniam B (1992): Humeral torsional deformity after supracondylar osteotomy for cubitus varus: its influence on the postosteotomy carrying angle. J Pediatr Orthop 12, 490493

Yamamoto I, Ishii S, Usui M, Ogino T, Kaneda K (1985): Cubitus varus deformity following supracondylar fracture of the humerus. A method for measuring rotational deformity. Clin Orthop 201, 179-185

Yoshida M, Saho Y, Katayose M (2010): Reliability of measuring humeral retroversion using ultrasound imaging in a healthy nonthrowing population. J Sport Rehabil $\underline{19}, 149-160$

K, Tillmann B: Anatomie. Springer Verlag, Heidelberg 2010

Zukunft-Huber B: Der kleine Fuß ganz groß: Dreidimensionale manuelle Fußtherapie bei kindlichen Fußfehlstellungen. 2. Auflage; Urban \& Fischer, München 2011

\section{Internetquellen:}

https://de.wikipedia.org/wiki/Nervus medianus _ _ /media/File:Gray812and814.svg; Zugriff am 20.08.2017

http://www.awmf.org/leitlinien/detail/11/012-014.html; Zugriff am 20.08.2017 


\section{Danksagung}

An dieser Stelle möchte ich zunächst Frau Prof. Dr. med. Anna-Kathrin Hell für die freundliche Überlassung des Themas dieser Arbeit und die hervorragende Unterstützung bei der Ausarbeitung dieser Schrift danken.

Ebenso möchte ich allen Kindern und ihren Eltern für die Teilnahme an unserer Untersuchung danken.

Mein Dank gilt auch Herrn Dr. med. Jan Christoph Katthagen für die kollegiale Zusammenarbeit und Austausch von Informationen während der Erstellung der Arbeit.

Für die Unterstützung bei der Erstellung von Statistiken, Tabellen und der Beantwortung von statistischen Fragestellungen möchte ich der Abteilung Medizinische Statistik der Universität Göttingen sowie Frau Dr. Claudia Harzer von der Technischen Universität Darmstadt meinen Dank aussprechen. Auch danke ich besonders Frau Dr. Lena Braunschweig von der Universität Göttingen für die wissenschaftliche Beratung und Beantwortung von Fragen rund um meine Doktorarbeit. 


\section{Lebenslauf}

Mein Name ist Claudia-Cornelia Gadomski, geborene Rudolph. Ich bin verheiratet, habe einen Sohn und wurde am 15. Dezember 1981 in Kassel als zweites Kind von Bärbel Rudolph und Dr.-Ing. Dr. med. Hans-Dieter Rudolph geboren.

Von 1988 -1992 habe ich die Grundschule in Kassel besucht. Daran schloss sich von 1992 -2001 der Besuch des Gymnasiums „Albert-Schweitzer-Schule“ in Kassel an, der mit dem Zeugnis über die allgemeine Hochschulreife 2001 beendet wurde.

Zum Wintersemester 2001 nahm ich das Studium der Humanmedizin an der Universität des Saarlandes in Homburg auf. Die ärztliche Vorprüfung legte ich im März 2004 ab. Danach erfolgte zum Sommersemester 2004 ein Wechsel an die Georg-August-Universität in Göttingen, an der ich im Sommer 2008 die ärztliche Prüfung ablegte.

Von 2008 bis September 2014 habe ich als Assistenzärztin am Westküstenklinikum in Heide gearbeitet. Die Prüfungen zur Fachärztin für Innere Medizin und zur Führung der Zusatzbezeichnung Notfallmedizin habe ich im Jahre 2014 abgelegt. Seit dieser Zeit bin ich im öffentlichen Gesundheitswesen tätig. 